DRAFT VERSION JANUARY 24, 2020

Typeset using LATEX twocolumn style in AASTeX61

\title{
UNRAVELLING THE COMPLEX BEHAVIOR OF MRK 421 WITH SIMULTANEOUS X-RAY AND VHE OBSERVATIONS DURING AN EXTREME FLARING ACTIVITY IN APRIL 2013
}

\section{MAGIC COLLABORATION:}

V. A. Acciari,${ }^{1}$ S. Ansoldi,${ }^{2,3}$ L. A. Antonelli,${ }^{4}$ A. Arbet Engels,${ }^{5}$ D. Bahck,${ }^{6}$ A. Babić, ${ }^{7}$ B. Banerjee,${ }^{8}$ U. Barres de Almeida, ${ }^{9}$ J. A. Barrio, ${ }^{10}$ J. Becerra González, ${ }^{1}$ W. Bednarek, ${ }^{11}$ L. Bellizzi,${ }^{12}$ E. Bernardini,${ }^{13,14}$ A. Berti ${ }^{15}$ J. Besenrieder,${ }^{16}$ W. Bhattacharyya,${ }^{13}$ C. Bigongiari,${ }^{4}$ A. Biland,${ }^{5}$ O. Blanch, ${ }^{17}$ G. Bonnoli,${ }^{12}$ Ž. Bošnjak,${ }^{7}$ G. Busetto,${ }^{14}$ R. Carosi,${ }^{18}$ G. Ceribella,${ }^{16}$ M. Cerruti,${ }^{19}$ Y. Chai,${ }^{16}$ A. Chilingarian,${ }^{20}$ S. Cikota,${ }^{7}$ S. M. Colak,${ }^{17}$ U. Colin,${ }^{16}$ E. Colombo, ${ }^{1}$ J. L. Contreras,${ }^{10}$ J. Cortina,${ }^{21}$ S. Covino, ${ }^{4}$ V. D'Elia,${ }^{4}$ P. Da Vela $,{ }^{18,}, 22$

F. Dazzi ${ }^{4}$ A. De Angelis, ${ }^{14}$ B. De Lotto,${ }^{2}$ F. Del Puppo, ${ }^{2}$ M. Delffino, ${ }^{17}, 23$ J. Delgado,${ }^{17,23}$ D. Depaoli,${ }^{15}$ F. Di Pierro, ${ }^{15}$ L. Di Venere, ${ }^{15}$ E. Do Souto Espiñeira,${ }^{17}$ D. Dominis Prester,${ }^{7}$ A. Donini,${ }^{2}$ D. Dorner,${ }^{24}$ M. Doro, ${ }^{14}$ D. Elsaesser,${ }^{6}$ V. Fallah Ramazani,${ }^{25}$ A. Fattorini,${ }^{6}$ G. Ferrara,${ }^{4}$ L. Foffano,${ }^{14}$ M. V. Fonseca,${ }^{10}$ L. Font,${ }^{26}$ C. Frude,${ }^{16}$ S. Fukami, ${ }^{3}$ R. J. García López, ${ }^{1}$ M. Garczarczyk,${ }^{13}$ S. Gasparyan, ${ }^{20}$ M. Gaug,${ }^{26}$ N. Giglietto ${ }^{15}$ F. Giordano, ${ }^{15}$ P. Gliwny, ${ }^{11}$ N. Godinović, ${ }^{7}$ D. Green, ${ }^{16}$ D. Hadasch ${ }^{3}$ A. Hahn,${ }^{16}$ J. Herrera, ${ }^{1}$ J. Hoang,${ }^{10}$ D. Hrupec, ${ }^{7}$ M. HÜtten, ${ }^{16}$ T. Inada,${ }^{3}$ S. Inoue,${ }^{3}$ K. Ishio,${ }^{16}$ Y. Iwamura,${ }^{3}$ L. Jouvin, ${ }^{17}$ Y. Kajiwara, ${ }^{3}$ D. Kerszberg,${ }^{17}$ Y. Kobayashi,${ }^{3}$ H. Kubo,${ }^{3}$ J. Kushida,${ }^{3}$ A. Lamastra,${ }^{4}$ D. Lelas,${ }^{7}$ F. Leone,${ }^{4}$ E. Lindfors,${ }^{25}$ S. Lombardi,${ }^{4}$ F. Longo, ${ }^{2,27}$ M. López,${ }^{10}$ R. López-Coto, ${ }^{14}$ A. López-Oramas, ${ }^{1}$ S. Loporchio, ${ }^{15}$

B. Machado de Oliveira Fraga, ${ }^{9}$ C. Maggio, ${ }^{26}$ P. Majumdar,${ }^{8}$ M. Makariev,${ }^{28}$ M. Mallamaci, ${ }^{14}$ G. Maneva, ${ }^{28}$ M. Manganaro, ${ }^{7}$ K. Mannheim, ${ }^{24}$ L. Maraschi ${ }^{4}$ M. Mariotti,${ }^{14}$ M. Martínez,${ }^{17}$ D. Mazin,${ }^{16,3}$ S. Mender,${ }^{6}$ S. Mićanović, ${ }^{7}$ D. Miceli,${ }^{2}$ T. Miener,${ }^{10}$ M. Minev, ${ }^{28} \mathrm{~J}$. M. Miranda,${ }^{12}$ R. Mirzoyan,${ }^{16}$ E. Molina,${ }^{19}$ A. Moralejo,${ }^{17}$ D. Morcuende ${ }^{10}$ V. Moreno,${ }^{26}$ E. Moretti,${ }^{17}$ P. Munar-Adrover,${ }^{26}$ V. Neustroev,${ }^{25}$ C. Nigro, ${ }^{13}$ K. Nilsson, ${ }^{25}$ D. Ninci,${ }^{17}$ K. Nishijima,${ }^{3}$ K. Noda,${ }^{3}$ L. Nogués,${ }^{17}$ S. Nozaki,${ }^{3}$ Y. Ohtani,${ }^{3}$ T. Oka,${ }^{3}$ J. Otero-Santos,${ }^{1}$ M. Palatiello,${ }^{2}$ D. Paneque,${ }^{16,3}$ R. Paoletti,${ }^{12}$ J. M. Paredes,${ }^{19}$ L. Pavletić, ${ }^{7}$ P. Peñil,${ }^{10}$ M. Peresano, ${ }^{2}$ M. Persic,${ }^{2,29}$ P. G. Prada Moroni,${ }^{18}$ E. Prandini,${ }^{14}$ I. Puljak,${ }^{7}$ W. Rhode,${ }^{6}$ M. Ribó,${ }^{19}$ J. Rico,${ }^{17}$ C. Righi, ${ }^{4}$

A. Rugliancich,${ }^{18}$ L. Saha,${ }^{10}$ N. Sahakyan,${ }^{20}$ T. Saito,${ }^{3}$ S. Sakurai,${ }^{3}$ K. Satalecka,${ }^{13}$ B. Schleicher,${ }^{24}$

K. Schmidt ${ }^{6}$ T. Schweizer,${ }^{16}$ J. Sitarek, ${ }^{11}$ I. Šnidarić, ${ }^{7}$ D. Sobczynska, ${ }^{11}$ A. Spolon, ${ }^{14}$ A. Stamerra,${ }^{4}$ D. Strom, ${ }^{16}$ M. Strzys,${ }^{3}$ Y. Suda,${ }^{16}$ T. Surić, ${ }^{7}$ M. Takahashi ${ }^{3}$ F. Tavecchio,${ }^{4}$ P. Temnikov ${ }^{28}$ T. Terzić, ${ }^{7}$ M. Teshima,${ }^{16,3}$

N. Torres-Albà, ${ }^{19}$ L. Tosti,${ }^{15}$ J. van Scherpenberg,${ }^{16}$ G. Vanzo,${ }^{1}$ M. Vazquez Acosta,${ }^{1}$ S. Ventura,${ }^{12}$ V. Verguilov ${ }^{28}$ C. F. Vigorito,${ }^{15}$ V. Vitale,${ }^{15}$ I. Vovk,${ }^{16}$ M. Will,${ }^{16}$ and D. Zarić ${ }^{7}$

OTHER GROUPS AND COLLABORATORS:

M. Petropoulou, ${ }^{30}$ J. Finke, ${ }^{31}$ F. D’Ammando, ${ }^{32}$ M. Baloković, ${ }^{33,34}$ G. Madejski,${ }^{35}$ K. Mori,${ }^{36}$ Simonetta Puccetti,${ }^{37}$ C. Leto,${ }^{38,39}$ M. Perri, ${ }^{38,39}$ F. Verrecchia,${ }^{38,39}$ M. Villata, ${ }^{40}$ C. M. Raiteri, ${ }^{40}$ I. Agudo,${ }^{41}$ R. Bachev, ${ }^{42}$ A. Berdyugin, ${ }^{43}$ D. A. Blinov,${ }^{44,45,46}$ R. Chanishvili,${ }^{47}$ W. P. Chen,${ }^{48}$ R. Chigladze,${ }^{47}$ G. Damljanovic ${ }^{49}$ C. Eswaraiah, ${ }^{48}$ T. S. Grishina, ${ }^{44}$ S. Ibryamov,${ }^{42}$ B. Jordan,${ }^{50}$ S. G. Jorstad,${ }^{44,51}$ M. Joshi,${ }^{44}$ E. N. Kopatskaya ${ }^{44}$ O. M. Kurtanidze ${ }^{47,52,53}$ S. O. Kurtanidze, ${ }^{47}$ E. G. Larionova, ${ }^{44}$ L. V. Larionova, ${ }^{44}$ V. M. Larionov, ${ }^{44,54}$ G. Latev,${ }^{42}$ H. C. Lin, ${ }^{48}$ A. P. Marscher,${ }^{51}$ A. A. Mokrushina,${ }^{44,54}$ D. A. Morozova, ${ }^{44}$ M. G. Nikolashvili,${ }^{47}$ E. Semkov,${ }^{42}$ P.S. Smith,${ }^{55}$ A. Strigachev, ${ }^{42}$ Yu. V. Troitskaya, ${ }^{44}$ I. S. Troitsky,${ }^{44}$ O. Vince, ${ }^{49}$ J. Barnes,${ }^{56}$ T. GÜver, ${ }^{57}$ J. W. Moody ${ }^{58}$ A. C. Sadun, ${ }^{59}$ T. Hovatta,${ }^{60,61}$ J. L. Richards, ${ }^{62}$ W. MaX-Moerbeck ${ }^{63}$ A. C. R. Readhead, ${ }^{64}$ A. Lähteenmäki ${ }^{61,65}$ M. Tornikoski,${ }^{61}$ J. TAmmi,${ }^{61}$ V. Ramakrishnan,${ }^{61}$ And R. ReinthaL ${ }^{43}$

${ }^{1}$ Inst. de Astrofsica de Canarias, E-38200 La Laguna, and Universidad de La Laguna, Dpto. Astrofsica, E-38206 La Laguna, Tenerife, Spain

${ }^{2}$ Universit di Udine, and INFN Trieste, I-33100 Udine, Italy

${ }^{3}$ Japanese MAGIC Consortium: ICRR, The University of Tokyo, 277-8582 Chiba, Japan; Department of Physics, Kyoto University, 606-8502 Kyoto, Japan; Tokai University, 259-1292 Kanagawa, Japan; RIKEN, 351-0198 Saitama, Japan

${ }^{4}$ National Institute for Astrophysics (INAF), I-00136 Rome, Italy

${ }^{5}$ ETH Zurich, CH-8093 Zurich, Switzerland 
${ }^{6}$ Technische Universitt Dortmund, D-44221 Dortmund, Germany

${ }^{7}$ Croatian Consortium: University of Rijeka, Department of Physics, 51000 Rijeka; University of Split - FESB, 21000 Split; University of Zagreb - FER, 10000 Zagreb; University of Osijek, 31000 Osijek; Rudjer Boskovic Institute, 10000 Zagreb, Croatia

${ }^{8}$ Saha Institute of Nuclear Physics, HBNI, 1/AF Bidhannagar, Salt Lake, Sector-1, Kolkata 700064, India

${ }^{9}$ Centro Brasileiro de Pesquisas Fsicas (CBPF), 22290-180 URCA, Rio de Janeiro (RJ), Brasil

${ }^{10}$ IPARCOS Institute and EMFTEL Department, Universidad Complutense de Madrid, E-28040 Madrid, Spain

${ }^{11}$ University of Lodz, Faculty of Physics and Applied Informatics, Department of Astrophysics, 90-236 Lodz, Poland

${ }^{12}$ Universit di Siena and INFN Pisa, I-53100 Siena, Italy

${ }^{13}$ Deutsches Elektronen-Synchrotron (DESY), D-15738 Zeuthen, Germany

${ }^{14}$ Universit di Padova and INFN, I-35131 Padova, Italy

${ }^{15}$ Istituto Nazionale Fisica Nucleare (INFN), 00044 Frascati (Roma) Italy

${ }^{16}$ Max-Planck-Institut für Physik, D-80805 München, Germany

${ }^{17}$ Institut de Física d'Altes Energies (IFAE), The Barcelona Institute of Science and Technology (BIST), E-08193 Bellaterra (Barcelona), Spain

${ }^{18}$ Universit di Pisa, and INFN Pisa, I-56126 Pisa, Italy

${ }^{19}$ Universitat de Barcelona, ICCUB, IEEC-UB, E-08028 Barcelona, Spain

${ }^{20}$ The Armenian Consortium: ICRANet-Armenia at NAS RA, A. Alikhanyan National Laboratory

${ }^{21}$ Centro de Investigaciones Energticas, Medioambientales y Tecnolgicas, E-28040 Madrid, Spain

${ }^{22}$ now at University of Innsbruck

${ }^{23}$ also at Port d'Informaci Cientfica (PIC) E-08193 Bellaterra (Barcelona) Spain

${ }^{24}$ Universität Würzburg, D-97074 Würzburg, Germany

${ }^{25}$ Finnish MAGIC Consortium: Finnish Centre of Astronomy with ESO (FINCA), University of Turku, FI-20014 Turku, Finland; Astronomy Research Unit, University of Oulu, FI-90014 Oulu, Finland

${ }^{26}$ Departament de Física, and CERES-IEEC, Universitat Autònoma de Barcelona, E-08193 Bellaterra, Spain

27 also at Dipartimento di Fisica, Università di Trieste, I-34127 Trieste, Italy

${ }^{28}$ Inst. for Nucl. Research and Nucl. Energy, Bulgarian Academy of Sciences, BG-1784 Sofia, Bulgaria

29 also at INAF-Trieste and Dept. of Physics \&5 Astronomy, University of Bologna

${ }^{30}$ Princeton University, Princeton NJ, USA

${ }^{31}$ US Naval Research Laboratory, Washington DC, USA

${ }^{32}$ INAFIstituto di Radioastronomia, Via P. Gobetti 101, I-40129 Bologna, Italy

${ }^{33}$ Center for Astrophysics | Harvard \& Smithsonian, 60 Garden Street, Cambridge, MA 02138, USA

${ }^{34}$ Black Hole Initiative at Harvard University, 20 Garden Street, Cambridge, MA 02138, USA

${ }^{35}$ W. W. Hansen Experimental Physics Laboratory, Kavli Institute for Particle Astrophysics and Cosmology, Department of Physics and SLAC National Accelerator Laboratory, Stanford University, Stanford, CA 94305, USA

${ }^{36}$ Columbia Astrophysics Laboratory, $550 \mathrm{~W}$ 120th St. New York, NY 10027, USA

${ }^{37}$ Agenzia Spaziale Italiana (ASI)-Unita' di Ricerca Scientifica, Via del Politecnico, I-00133 Roma, Italy

${ }^{38}$ ASI Science Data Center, Via del Politecnico snc I-00133, Roma, Italy

${ }^{39}$ INAF - Osservatorio Astronomico di Roma, via di Frascati 33, I-00040 Monteporzio, Italy

${ }^{40}$ INAF - Osservatorio Astrofisico di Torino, 10025 Pino Torinese (TO), Italy

${ }^{41}$ Instituto de Astrofísica de Andalucía (CSIC), Apartado 3004, E-18080 Granada, Spain

${ }^{42}$ Institute of Astronomy and National Astronomical Observatory, Bulgarian Academy of Sciences, 72 Tsarigradsko shosse Blvd., 1784 Sofia, Bulgaria

${ }^{43}$ Tuorla Observatory, Department of Physics and Astronomy, Väisäläntie 20, FIN-21500 Piikkiö, Finland

${ }^{44}$ Astronomical Institute, St. Petersburg State University, Universitetskij Pr. 28, Petrodvorets, 198504 St. Petersburg, Russia

${ }^{45}$ Department of Physics and Institute for Plasma Physics, University of Crete, 71003, Heraklion, Greece

${ }^{46}$ Foundation for Research and Technology - Hellas, IESL, Voutes, 71110 Heraklion, Greece

${ }^{47}$ Abastumani Observatory, Mt. Kanobili, 0301 Abastumani, Georgia

${ }^{48}$ Graduate Institute of Astronomy, National Central University, 300 Zhongda Road, Zhongli 32001, Taiwan

${ }^{49}$ Astronomical Observatory, Volgina 7, 11060 Belgrade, Serbia

${ }^{50}$ School of Cosmic Physics, Dublin Institute For Advanced Studies, Ireland

${ }^{51}$ Institute for Astrophysical Research, Boston University, 725 Commonwealth Avenue, Boston, MA 02215

${ }^{52}$ Engelhardt Astronomical Observatory, Kazan Federal University, Tatarstan, Russia

${ }^{53}$ Center for Astrophysics, Guangzhou University, Guangzhou 510006, China

${ }^{54}$ Pulkovo Observatory, St.-Petersburg, Russia 
${ }^{55}$ Steward Observatory, University of Arizona, 933 N. Cherry Ave., Tucson, AZ 85721, USA

${ }^{56}$ Department of Physics, Salt Lake Community College, Salt Lake City, Utah 84070 USA

${ }^{57}$ Istanbul University, Science Faculty, Department of Astronomy and Space Sciences, Beyazt, 34119, Istanbul, Turkey

${ }^{58}$ Department of Physics and Astronomy, Brigham Young University, Provo Utah 84602 USA

${ }^{59}$ Department of Physics, University of Colorado Denver, Denver, Colorado, CO 80217-3364, USA

${ }^{60}$ Finnish Centre for Astronomy with ESO (FINCA), University of Turku, FI-20014 Turku, Finland

${ }^{61}$ Aalto University, Metsähovi Radio Observatory, Metsähovintie 114, 02540 Kylmälä, Finland

${ }^{62}$ Department of Physics and Astronomy, Purdue University, West Lafayette, IN 47907, USA

${ }^{63}$ Departamento de Astronomia, Universidad de Chile, Camino El Observatorio 1515, Las Condes, Santiago, Chile

${ }^{64}$ Owens Valley Radio Observatory, California Institute of Technology, Pasadena, CA 91125, USA

${ }^{65}$ Aalto University Department of Radio Science and Engineering, P.O. BOX 13000, FI-00076 Aalto, Finland

\section{ABSTRACT}

We report on a multi-band variability and correlation study of the TeV blazar Mrk 421 during an exceptional flaring activity observed from 2013 April 11 to 2013 April 19. The study uses, among others, data from GASP-WEBT, Swift, NuSTAR, Fermi-LAT, VERITAS, and MAGIC. The large blazar activity, and the 43 hours of simultaneous NuSTAR and MAGIC/VERITAS observations, permitted variability studies on 15 minute time bins, and over three $\mathrm{X}$-ray bands (3-7 keV, 7-30 keV and 30-80 keV) and three very-high-energy ( $>0.1 \mathrm{TeV}$, hereafter VHE) gamma-ray bands $(0.2-0.4 \mathrm{TeV}, 0.4-0.8 \mathrm{TeV}$ and $>0.8 \mathrm{TeV})$. We detected substantial flux variations on multi-hour and subhour timescales in all the X-ray and VHE gamma-ray bands. The characteristics of the sub-hour flux variations are essentially energy-independent, while the multi-hour flux variations can have a strong dependence on the energy of the X-ray and the VHE gamma rays. The three VHE bands and the three X-ray bands are positively correlated with no time-lag, but the strength and the characteristics of the correlation changes substantially over time and across energy bands. Our findings favour multi-zone scenarios for explaining the achromatic/chromatic variability of the fast/slow components of the light curves, as well as the changes in the flux-flux correlation on day-long timescales. We interpret these results within a magnetic reconnection scenario, where the multi-hour flux variations are dominated by the combined emission from various plasmoids of different sizes and velocities, while the sub-hour flux variations are dominated by the emission from a single small plasmoid moving across the magnetic reconnection layer.

Keywords: BL Lacertae objects: individual (Markarian 421) galaxies: active gamma rays: general radiation mechanisms: nonthermal $\mathrm{X}$-rays: galaxies 


\section{INTRODUCTION}

Markarian 421 (Mrk 421), with a redshift of $z=0.0308$, is one of the closest BL Lac objects (Ulrich et al. 1975), which happens to be also the first BL Lac object significantly detected at gamma-ray energies (with EGRET, Lin et al. 1992), and the first extragalactic object significantly detected at very-high-energy $(>0.1 \mathrm{TeV}$, hereafter VHE) gamma rays (with Whipple, Punch et al. 1992). Mrk 421 is also the brightest persistent $\mathrm{X}$-ray/TeV blazar in the sky, and among the few sources whose spectral energy distribution (SED) can be accurately characterized by current instruments from radioto-VHE (Abdo et al. 2011). Consequently, Mrk 421 is among the few $\mathrm{X}$-ray/TeV objects that can be studied with a great level of detail during both low and high activity (Fossati et al. 2008; Aleksić et al. 2015b; Baloković et al. 2016), and hence an object whose study maximizes our chances to understand the blazar phenomenon in general.

Because of these reasons, every year since 2009, we organize extensive multiwavelength (MWL) observing campaigns where Mrk 421 is monitored from radio-toVHE gamma rays during the half year that it is visible with optical telescopes and Imaging Atmospheric Cherenkov Telescopes (IACTs). This multi-instrument and multi-year program provides a large time and energy coverage that, owing to the brightness and proximity of Mrk 421, yields the most detailed characterization of the broadband SED and its temporal evolution, compared to any other MWL campaign on any other TeV target.

During the MWL campaign in the 2013 season, in the second week of 2013 April, we observed exceptionally high X-ray and VHE gamma-ray activity with the Neil Gehrels Swift Gamma-ray Burst Observatory (Swift), the Nuclear Spectroscopic Telescope Array $(N u S T A R)$, the Large Area Telescope onboard the Fermi Gamma-ray Space Telecope (Fermi-LAT), the Major Atmospheric Gamma Imaging Cherenkov telescope (MAGIC), and the Very Energetic Radiation Imaging Telescope Array System (VERITAS), as reported in various Astronomer's Telegrams (e.g. see Baloković et al. 2013; Cortina \& Holder 2013; Paneque et al. 2013). Among other things, the VHE gammaray flux was found to be two orders of magnitude larger than that measured during the first months of the MWL campaign in January and February 2013 (Baloković et al. 2016). This enhanced activity triggered very deep observations with optical, X-ray and gamma-ray instruments, including a modified survey mode for Fermi from April 12 (23:00 UTC) until April 15th (18:00 UTC), which increased the LAT exposure on Mrk 421 by about a factor of two.
While Mrk 421 has shown outstanding X-ray and VHE gamma-ray activity in the past (e.g. Gaidos et al. 1996; Fossati et al. 2008; Abeysekara et al. 2020), this is the most complete characterization of a flaring activity of Mrk 421 to date. An extensive multi-instrument dataset was accumulated during nine consecutive days. It includes VHE observations with MAGIC, the use of public VHE data from VERITAS, and high-sensitive X-ray observations with $N u S T A R$. Notably, there are 43 hours of simultaneous VHE gamma-ray (MAGIC and VERITAS) and X-ray $(N u S T A R)$ observations. A first evaluation of the X-ray activity measured with Swift and NuSTAR was reported in Paliya et al. (2015). This manuscript reports the full multi-band characterisation of this outstanding event, which includes, for the first time, a report of the VHE gamma-ray data, and it focuses on an unprecedented study of the X-ray-vs-VHE correlation in $3 \times 3$ energy bands. This study demonstrates that there is a large degree of complexity in the variability in the $\mathrm{X}$-ray and VHE gamma-ray domains, which relate to the most energetic and variable segments of Mrk 421's SED, and indicates that the broadband emission of blazars require multi-zone theoretical models.

This paper is organized as follows: in Section 2 we briefly describe the observations that were performed, and in Section 3 we report the measured multiinstrument light curves. Section 4 provides a detailed characterization of the multi-band variability, with special focus on the X-ray and VHE gamma-ray variations observed on April 15th. In Section 5 we characterize the multi-band correlations observed when comparing the $\mathrm{X}$-ray emission in three energy bands, with that of VHE gamma rays in three energy bands. In Section 6 we discuss the implications of the observational results reported in this paper and finally, in Section 7, we provide some concluding remarks.

\section{OBSERVATIONS AND DATASETS}

The observations presented here are part of the multiinstrument campaign for Mrk421 that has occurred yearly since 2009 (Abdo et al. 2011). The instruments that participate in this campaign can change somewhat from year to year, but they are typically more than 20 covering energies from radio to VHE gamma rays. The 2013 campaign included observations from $N u S T A R$ for the first time, as a part of its primary mission (Harrison et al. 2013). The instruments that participated in the 2013 campaign, as well as their performances and data analysis strategies, were reported in Baloković et al. (2016), which is our first publication with the 2013 multi-instrument data set, and focused on the low $\mathrm{X}$ ray/VHE activity observed in January-to-March 2013. 
Table 1. MAGIC, VERITAS and NuSTAR Observations Overlap

\begin{tabular}{|c|c|c|c|c|c|c|}
\hline \multirow{3}{*}{$\begin{array}{l}\text { Night } \\
\text { (1) }\end{array}$} & \multirow{3}{*}{$\begin{array}{c}\text { Date } \\
\text { Apr } 2013 \\
(2)\end{array}$} & \multirow{3}{*}{$\begin{array}{l}\text { Date } \\
\text { MJD } \\
(3)\end{array}$} & \multicolumn{2}{|c|}{ MAGIC + VERITAS } & \multirow{3}{*}{$\begin{array}{c}N u S T A R \\
\operatorname{bins}^{\mathrm{a}} \\
(6)\end{array}$} & \multirow{3}{*}{$\begin{array}{c}\text { VHE observations with } \\
\text { simultaneous X-ray coverage } \\
(7)\end{array}$} \\
\hline & & & bins $^{a}$ & simultaneous $^{\mathrm{b}}$ & & \\
\hline & & & (4) & $(5)$ & & \\
\hline $1 \ldots$ & $10 / 11$ & $56392 / 56393$ & $21+15$ & 0 & 43 & $30 / 36(83 \%)$ \\
\hline $2 \ldots$ & $11 / 12$ & $56393 / 56394$ & $24+25$ & 2 & 65 & $33 / 47(70 \%)$ \\
\hline $3 \ldots$ & $12 / 13$ & $56394 / 56395$ & $23+14$ & 4 & 17 & $14 / 33(42 \%)$ \\
\hline $4 \ldots$ & $13 / 14$ & $56395 / 56396$ & $25+19$ & 3 & 30 & $29 / 41(71 \%)$ \\
\hline $5 \ldots$ & $14 / 15$ & $56396 / 56397$ & $25+24$ & 2 & 30 & $31 / 47(66 \%)$ \\
\hline $6 \ldots$ & $15 / 16$ & $56397 / 56398$ & $16+11$ & 0 & 30 & $20 / 27(74 \%)$ \\
\hline $7 \ldots$ & $16 / 17$ & $56398 / 56399$ & $10+0$ & 0 & 32 & $4 / 10(40 \%)$ \\
\hline $8 \ldots$ & $17 / 18$ & $56399 / 56400$ & $13+0$ & 0 & 20 & $6 / 13(46 \%)$ \\
\hline $9 \ldots$ & $18 / 19$ & $56400 / 56401$ & $10+0$ & 0 & 19 & $6 / 10(60 \%)$ \\
\hline all... & $10-19$ & $56392-56401$ & $167+108$ & 11 & 286 & $173 / 264(66 \%)$ \\
\hline
\end{tabular}

$a_{\text {Number of }}$ 15-minute time bins with observations by the respective instrument.

${ }^{b}$ Number of 15-minute time bins with measurements above $0.4 \mathrm{TeV}$ in which MAGIC and VERITAS observed the source simultaneously.

${ }^{c}$ The ratio of X-ray 15-bins simultaneous to the VHE 15-min bins (used as denominator), and percentage.

During the first observations in April 2013, Mrk 421 showed high X-ray and VHE gamma-ray activity, which triggered daily-few-hour-long multi-instrument observations that lasted from April 10 (MJD 56392) to April 19 (MJD 56401). Among other instruments, this data set contains an exceptionally deep temporal coverage at VHE gamma rays above $0.2 \mathrm{TeV}$, as the source was observed with MAGIC during nine consecutive nights, and with VERITAS during six nights (Benbow 2017). The geographical longitude of VERITAS is $93^{\circ}$ (about six hours) west to that of MAGIC, and hence VERITAS observations followed those from MAGIC, sometimes providing continuous VHE gamma-ray coverage during 10 hours in a single night. The total MAGIC observation time was nearly 42 hours, while for VERITAS it was 27 hours, yielding a total VHE observation time of 69 hours in nine days (66 hours when counting once the MAGIC-VERITAS simultaneous observations). The time coverage of VHE data is slightly different for different VHE gamma-ray energies, being a few hours longer above $0.8 \mathrm{TeV}$ in comparison to that below $0.4 \mathrm{TeV}$. This un-even coverage is due to the increased energy threshold associated with observations taken at large zenith angles. In the case of MAGIC, the energy threshold at zenith angles of about $60^{\circ}$ is about
0.4 TeV, and hence the low-energy gamma-ray observations are not possible (see Aleksić et al. 2016, for dependence of analysis energy threshold with the zenith angle of observations). MAGIC and VERITAS observed Mrk 421 simultaneously during $2 \mathrm{hr} 45 \mathrm{~min}$. The simultaneous observations between these two instruments occurred when MAGIC was observing at large zenith angle $\left(>55^{\circ}\right)$, hence yielding simultaneous flux measurements only above $0.4 \mathrm{TeV}$. The extensive VHE coverage is particularly relevant since, as it will be described in Section 3, Mrk 421 showed large variability and one of the brightest VHE flaring activity recorded to date. This unprecedented brightness allows us to match the sampling frequency of the simultaneous VHE (MAGIC and VERITAS) and X-ray (NuSTAR) observations to 15-min time intervals in three distinct energy bands: $0.2-0.4 \mathrm{TeV}, 0.4-0.8 \mathrm{TeV}$ and $>0.8 \mathrm{TeV}$. The abovementioned time-cadence and energy bands were chosen as a good compromise between having both, a good sampling of the multi-band VHE activity of Mrk 421 during the April 2013 period, and reasonably accurate VHE flux measurements, with the relative flux errors typically below $10 \%$. See Benbow (2017) for the VERITAS photon fluxes. In the case of the MAGIC light curves in the three energy bands, the small effect related to 
the event migration in energy was computed using the VHE gamma-ray spectrum from the full 9-day data set, which is well represented by the following log-parabola function

$$
\frac{d \Phi}{d E}=\left(\frac{E}{0.3 \mathrm{TeV}}\right)^{-(2.14)-(0.45) \cdot \log 10\left(\frac{E}{0.3 \mathrm{TeV}}\right)}
$$

However, owing to the relatively narrow energy bands, the derived photon fluxes are not significantly affected by the specific choice of the used spectral shape: the photon fluxes derived with power-law spectra with indices $p=2$ and $p=3$ are in agreement, within the statistical uncertainties, with those derived with the 9day log-parabolic spectral shape.

Using the simultaneous MAGIC and VERITAS observations, we noted a systematic offset of about $20 \%$ in the VHE gamma-ray flux measurements derived with these two instruments. The VHE gamma-ray fluxes from VERITAS are systematically lower than those from MAGIC by a factor that is energy-dependent, being about $10 \%$ in the $0.2-0.4 \mathrm{TeV}$ band, and about $30 \%$ above $0.8 \mathrm{TeV}$. This offset, which is perfectly consistent with the known systematic uncertainties affecting each experiment (Madhavan \& for the VERITAS Collaboration 2013; Aleksić et al. 2016), becomes evident due to the low statistical uncertainties associated with the flux measurements reported here. Appendix A reports a characterization of this offset, and describes the procedure that we followed for correcting it, scaling up the VERITAS fluxes to match those from MAGIC. The physics results reported in this manuscript do not depend on the absolute value of the VHE gamma-ray flux, and hence one could have scaled down the MAGIC fluxes to match those of VERITAS. The correction applied is only relevant for the intra-night variability and correlation studies.

A key characteristic of this dataset is the extensive and simultaneous coverage in the X-ray bands provided by Swift and, especially, by NuSTAR. Swift observed Mrk 421 for 18 hours, split in 63 observations spread over the nine days, and performed during the MAGIC and VERITAS observations. NuSTAR observed Mrk 421 for 71 hours during the above-mentioned nine days, out of which 43 hours were taken simultaneously to the VHE observations from MAGIC and VERITAS. The VHE and X-ray temporal coverage is summarized in Table 1.

The raw NuSTAR data were processed exactly as described in Baloković et al. (2016), except that in this study the NUSTAR analysis was performed separately for each 15-minute time bin with simultaneous VHE observations, as summarized in Table 1. Using Xspec (Arnaud 1996), we calculated fluxes in the $3-7 \mathrm{keV}$,
7-30 keV, and $30-80 \mathrm{keV}$ bands from a fit of a logparabolic model to the data within each time bin. The cross-normalization between the two NuSTAR telescope modules was treated as a free parameter. The statistical uncertainties on fluxes were calculated as $68 \%$ confidence intervals, and do not include the systematic uncertainty in absolute calibration, which is estimated to be 10-20\% (Madsen et al. 2015).

The analysis procedures used to process the SwiftXRT data are described in Baloković et al. (2016). In addition, in order to avoid additional flux uncertainties, we excluded 16 Swift-XRT observations in which Mrk 421 was positioned near the CCD bad-columns (Madsen et al. 2017). Fig. 1 shows a comparison of the Swift-XRT and NuSTAR X-ray fluxes in the band $3-7 \mathrm{keV}$. Overall, there is a good agreement between the two instruments, with flux differences typically smaller than $20 \%$. Such flux differences are within the systematic uncertainties in the absolute flux calibration of NuSTAR (Madsen et al. 2015) and Swift-XRT (Madsen et al. 2017).

Differently to the Fermi-LAT analysis reported in Baloković et al. (2016), the LAT data results shown here were produced with events above $0.3 \mathrm{GeV}$ (instead of $0.1 \mathrm{GeV}$ ) and with Pass8 (instead of Pass7). The analysis above $0.3 \mathrm{GeV}$ is less affected by systematic uncertainties, and it is also less sensitive to possible contamination from non-accounted (transient) neighboring sources. The higher minimum energy reduces somewhat the detected number of photons from the source, but, owing to its hard gamma-ray spectrum (photon index $<2.0$ ), the effect is small. Specifically, we used the standard Fermi analysis software tools version v11r07p00, and the P8R3_SOURCE_V2 response function on events with energy above $0.3 \mathrm{GeV}$ coming from a $10^{\circ}$ region of interest (ROI) around Mrk 421. We used a $100^{\circ}$ zenith-angle cut to avoid contamination from the Earth's limb ${ }^{1}$, and modeled the diffuse Galactic and isotropic extragalactic background with the files gll_iem_v07.fits and iso_P8R3_SOURCE_V2_v1.txt respectively ${ }^{2}$. All point sources in the fourth Fermi-LAT source catalog (4FGL, Abdollahi et al. 2019) located in the $10^{\circ} \mathrm{ROI}$ and an additional surrounding $5^{\circ}$-wide annulus were included in the model. In the unbinned likelihood fit, the spectral parameters were set to the values from the $4 \mathrm{FGL}$, while the normalization of the diffuse components and the normalization parameters

\footnotetext{
1 A zenith-angle cut of $90^{\circ}$ is needed if using events down to $0.1 \mathrm{GeV}$, but one can use a zenith-angle cut of $100^{\circ}$ above $0.3 \mathrm{GeV}$ without the need for using a dedicated Earth limb template.

2 https://fermi.gsfc.nasa.gov/ssc/data/access/lat/ BackgroundModels.html
} 
of the 16 sources (within the ROI) identified as variable were initially left free to vary. However, owing to the short timescales considered in this analysis, only two of these sources were significantly detected in 10 days: 4FGL J1127.8+3618 and 4FGL J1139.0+4033, and hence we fixed the normalization of the other ones to the 4FGL catalog values. The Fermi-LAT spectrum from the 10-day time period considered here (from MJD 56392 to MJD 56402) is well described with a power-law function with a photon flux above $0.3 \mathrm{GeV}$ of $(23.3 \pm 1.6) \times 10^{-8} \mathrm{~cm}^{-2} \mathrm{~s}^{-1}$ and photon index $1.79 \pm 0.05$. A spectral analysis over 1-day and 12-hour time intervals shows that the photon index does not vary significantly throughout the 10-day period. The data was split into 12-hours-long intervals centered at the VHE observations (e.g. simultaneous to the VHE) and their complementary time intervals (e.g. when there are no VHE observations), which are close to 12-hours-long intervals. Owing to the limited event count in the 12-hour time intervals, and the lack of spectral variability throughout the 10-day period, we fixed the shape to the power-law index to 1.79 (the value from the 10-day period) to derive the photon fluxes, keeping always the normalization factor for Mrk 421 and the two above-mentioned 4FGL sources as a free parameters in the log-likelihood fit of each of the 12-hour time intervals.

The characterization of the activity of Mrk 421 at optical frequencies was performed with many instruments from the GLAST-AGILE Support Program (GASP) of the Whole Earth Blazar Telescope (WEBT), here after GASP-WEBT (e.g., Villata et al. 2008, 2009), namely the observatory in Roque de los Muchachos (KVA telescope), Lowell (Perkins telescope), Crimean, St. Petersburg, Abastumani, Rozhen $(50 / 70 \mathrm{~cm}, 60 \mathrm{~cm}$, and 200 $\mathrm{cm}$ telescopes), Vidojevica, and Lulin. Moreover, this study also uses data from the iTelescopes, the Remote Observatory for Variable Object Research (ROVOR), and the TUBITAK National Observatory (TUG). The polarization measurements were performed with four observatories: Lowell (Perkins telescope), St. Petersburg, Crimean, and Steward (Bok telescope). The data reduction was done exactly as in Baloković et al. (2016).

Besides the $15 \mathrm{GHz}$ and $37 \mathrm{GHz}$ radio observations performed with the OVRO and Metsahovi telescopes, which were described in Baloković et al. (2016), here we also present a flux measurement performed with the IRAM $30 \mathrm{~m}$ telescope at $86 \mathrm{GHz}$. This observation was performed under the Polarimetric Monitoring of AGN at Millimeter Wavelengths program (POLAMI ${ }^{3}$, Agudo et

\footnotetext{
${ }^{3}$ http://polami.iaa.es
}

al. 2018b), that regularly monitors Mrk 421 in the short millimeter range. The POLAMI data was reduced and calibrated as described in Agudo et al. (2018a).

\section{MULTI-INSTRUMENT LIGHT CURVES DURING THE OUTSTANDING FLARING ACTIVITY IN APRIL 2013}

The multi-instrument light curves derived from all the observations spanning from radio to VHE gamma rays are shown in Fig. 1. The top panel of Fig. 1 shows an excellent coverage of the 9-day flaring activity in the VHE regime, as a result of the combined MAGIC and VERITAS observations. The peak flux at $\mathrm{TeV}$ energies, observed in April 13 (MJD 56395), reached up to 15 times the flux of the Crab Nebula, that is about 30 times the typical non-flaring activity of Mrk 421, and about 150 times the activity shown a few months before, on January and February 2013, as reported in Baloković et al. (2016). Moreover, this is the highest TeV flux ever measured with MAGIC for any blazar. This is also the third highest flux ever measured from a blazar with an IACT, after the extremely large outburst from Mrk 421 detected with VERITAS in February 2010 (Abeysekara et al. 2020) and the large flare from PKS 2155-304 detected by HESS in July 2006 (Aharonian et al. 2007).

Figure 1 shows that the most extreme flux variations occur in the X-ray and the VHE gamma-ray bands. At $\mathrm{GeV}$ energies, within the accuracy of the measurements, there is enhanced activity only on MJD 56397 (April 15th), when the flux is about a factor of two larger than the previous and following $\sim 12$-hour time intervals. Interestingly, on April 15th we also find the highest Xray flux, and the highest intra-night $\mathrm{X}$-ray flux increase measured during this flaring activity in April 2013.

The R-band activity is comparable to the one measured in January-March 2013, when Mrk 421 showed very low VHE and X-ray activity (Baloković et al. 2016). The measured fluxes at optical wavelengths are large when compared to the flux levels typically seen during the period of 2007-2015 (Carnerero et al. 2017). Generally, during the observations performed in January-April 2013, Mrk 421 was 4-5 times brighter in the optical than the photometric minima that occurred in 2008-09 and at the end of 2011. Fig. 1 shows that Mrk 421 faded at R-band from about $60 \mathrm{mJy}$ on MJD 56393 to about 45 mJy two days later, and then varied between 45-50 mJy during the following week, and appear decoupled from the VHE and X-ray activity. The optical light curve is in agreement with that of the less well sampled Swift/UVOT light curve. Besides the optical brightness of Mrk 421 in 2013, the object showed a bluer optical continuum than average. This 


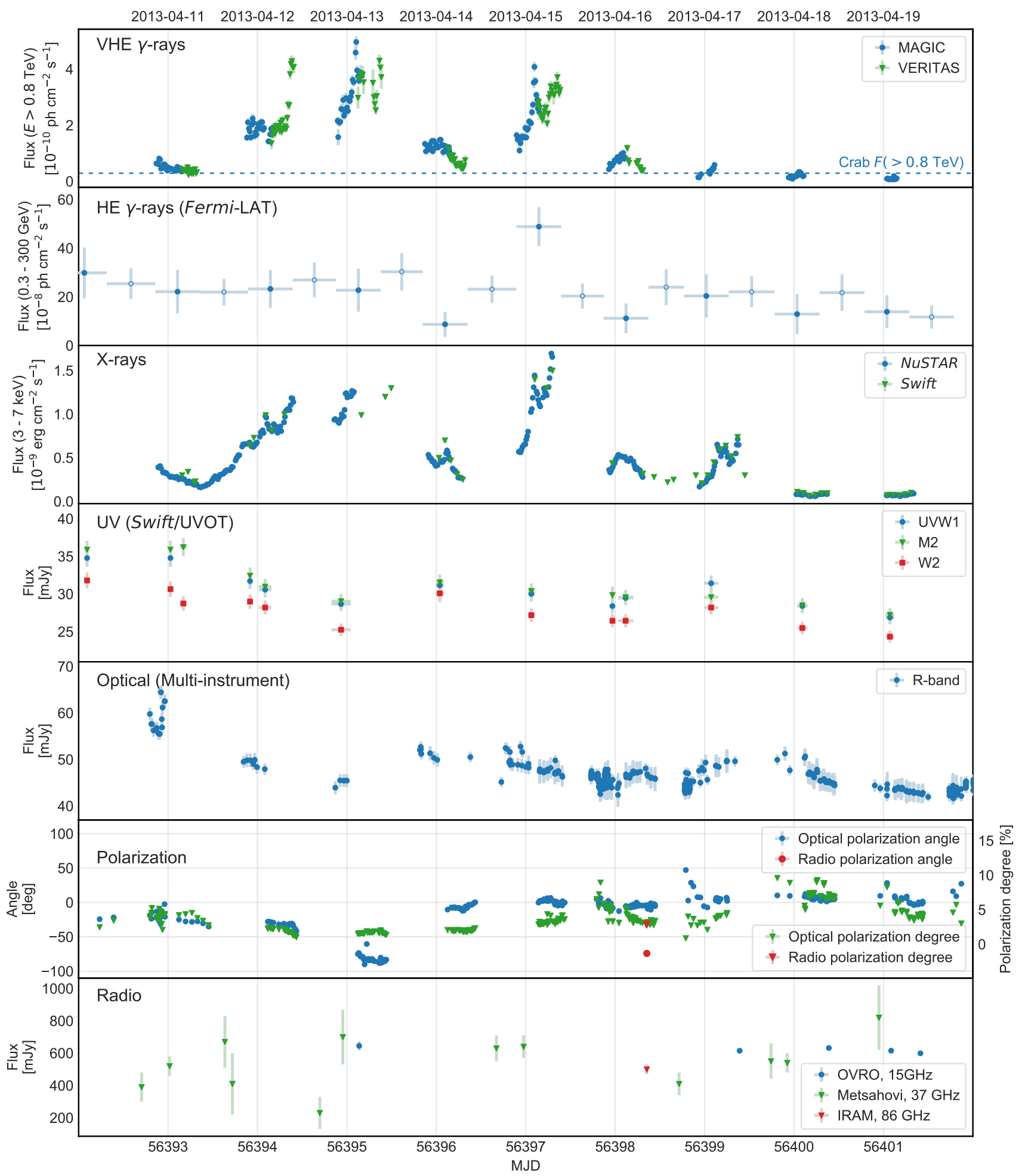

Figure 1. Multiwavelength light curve for Mrk 421 during the bright flaring activity in April 2013. The correspondence between the instruments and the measured fluxes is given in the legends. The horizontal dashed line in the VHE light curves represents the flux of the Crab Nebula, as reported in Aleksić et al. (2016). The VERITAS fluxes have been scaled using the coefficients described in Appendix A. The filled markers in the Fermi-LAT panel depict the flux during the 12-hour time interval centered at the VHE observations, while the open markers denote the periods without corresponding observations in VHE bands. 
was determined from the differential spectrophotometry obtained by the Steward Observatory monitoring program. By comparing the instrumental spectrum of Mrk 421 with that of a nearby field comparison star, it is found that for the wavelengths $475 \mathrm{~nm}$ and $725 \mathrm{~nm}$ $[F(475) / F(725)]_{\text {April-2013 }} /[F(475) / F(725)]_{\text {average }}=$ $1.072 \pm 0.002$, where the average instrumental flux ratio is determined from all of the available observations from 2008-2018. The bluer color of Mrk 421 is consistent with a higher dominance of the non-thermal continuum over the host galaxy starlight included within the observing aperture, which has a redder spectrum. This explanation for the observed variations in the optical color of Mrk 421 is further confirmed by the trend that the continuum becomes slightly redder as the AGN generally fades during April 2013. The same trend in color is also seen in the long-term near-IR data (Carnerero et al. 2017).

Optical linear polarization of Mrk 421 was also monitored, and the measurements are shown in Fig. 1. Again, the results are comparable to those measured during the first quarter in 2013, and reported in Baloković et al. (2016). Since 2008, the degree of polarization, $P$, has ranged from $0 \%$ to $15 \%$, although observations of $P>10 \%$ are rare, about 10 out of around 1400 observations (Carnerero et al. 2017). During April 2013, the polarization ranged from about $1-9 \%$, with a large majority of measurements showing $P<5 \%$. The largest changes in the degree of polarization on a daily time scale were an increase from $P \sim 3 \%$ to $P \sim 7 \%$ on MJD 56399/400, followed by a decrease back to about $4 \%$ on the next day. Changes of nearly as much as $5 \%$ in polarization are observed within a day, particularly on MJD 56398/99, but otherwise, variations in $P$ are typically limited to $<1 \%$ over hour timescales. The electric vector position angle (EVPA) of the optical polarization was at about $-20^{\circ}$ at the start of 2013 April. Between MJD 56394 and MJD 56395 the EVPA rotated from about $-30^{\circ}$ to about $-90^{\circ}$ while generally $P<2.5 \%$. The largest daily rotation in EVPA occurs between MJD 56395 and MJD 56396, where the EVPA goes from about $-90^{\circ}$ to about $-10^{\circ}$. Because of the daily gap in the optical monitoring, it is unfortunately not clear if the EVPA reversed its direction of rotation from MJD 56394 to MJD 56396 (i.e. 2 days), or continued in the same direction requiring a rotation of $>90^{\circ}$ during one of the two observing gaps on MJD 56394/6. The variability of Mrk 421 during the densely sampled portions of the optical monitoring does not hint that such large changes in EVPA can take place on short time scales until near the the end of MJD 56398 when a counterclockwise rotation of about $50^{\circ}$ is seen over a period of about 6 hours. Outside of this excursion, the EVPA stays near $0^{\circ}$ from MJD 56396 onward. The single daily deviation of EVPA to $90-100^{\circ}$ on MJD 56395 coincides with brightest VHE flare observed in April 2013. However, no significant change in EVPA is apparent during the sharp rise in VHE flux observed near the middle of MJD 56394 or during the dramatic high-energy activity at the beginning of MJD 56397. For most of the monitoring period, the optical EVPA was near the historical most likely angle for this object $\left(\mathrm{EVPA}=0^{\circ}\right.$, Carnerero et al. 2017), although the one-day excursion on MJD 56395 brought the EVPA nearly orthogonal to the most likely value. For comparison, the $15 \mathrm{GHz}$ VLBI maps of Mrk 421 show a jet detected out to about 5 mas at the position angle of about $-40^{\circ}$ (Lister et al. 2019). In the radio band the activity measured during the entire nine-day observing period is constant with a flux of about $0.6 \mathrm{Jy}$. The single $86 \mathrm{GHz}$ measurement with IRAM $30 \mathrm{~m}$ shows a polarization degree of about $3 \%$, which is similar to that of the optical frequencies; yet the polarization angle differs by about $70^{\circ}$, which suggests that the optical and radio emission are being produced in different locations of the jet of Mrk 421 . Overall, the radio and optical fluxes, as well the optical polarization variations (polarization degree and EVPA) appear completely decoupled from the large X-ray and VHE gamma-ray activity seen in April 2013. In fact, the behavior observed at radio and optical during April 2013 is similar to the one observed during the previous months, when Mrk 421 showed extremely low X-ray and VHE gamma-ray activity (see Baloković et al. 2016).

The hard X-ray and the VHE gamma-ray bands covered with NuSTAR, MAGIC, and VERITAS are the most interesting ones because they exhibit the largest flux variations, and because of the exquisite temporal coverage and the simultaneity in the dataset. Fig. 2 reports the flux measurements in these bands, each split into three distinct bands, $3-7 \mathrm{keV}, 7-30 \mathrm{keV}$ and 30 $80 \mathrm{keV}^{4}$ for NuSTAR and $0.2-0.4 \mathrm{TeV}, 0.4-0.8 \mathrm{TeV}$ and $>0.8 \mathrm{TeV}$ for MAGIC and VERITAS. The temporal coverage for the band $>0.8 \mathrm{TeV}$ is a about 2 hours longer than for the band $0.2-0.4 \mathrm{TeV}$ because of the increasing analysis energy threshold with the increasing zenith angle of the observations. The exquisite characterization of the multi-band flux variations in the X-ray and VHE gamma-ray bands reported in Fig. 2 will be used in the next sections for the broadband variability and correlation studies.

\footnotetext{
4 The upper edge of the NuSTAR energy range is actually $79 \mathrm{keV}$, but owing to the negligible impact on the flux values, in this paper we will use $80 \mathrm{keV}$ for simplicity.
} 


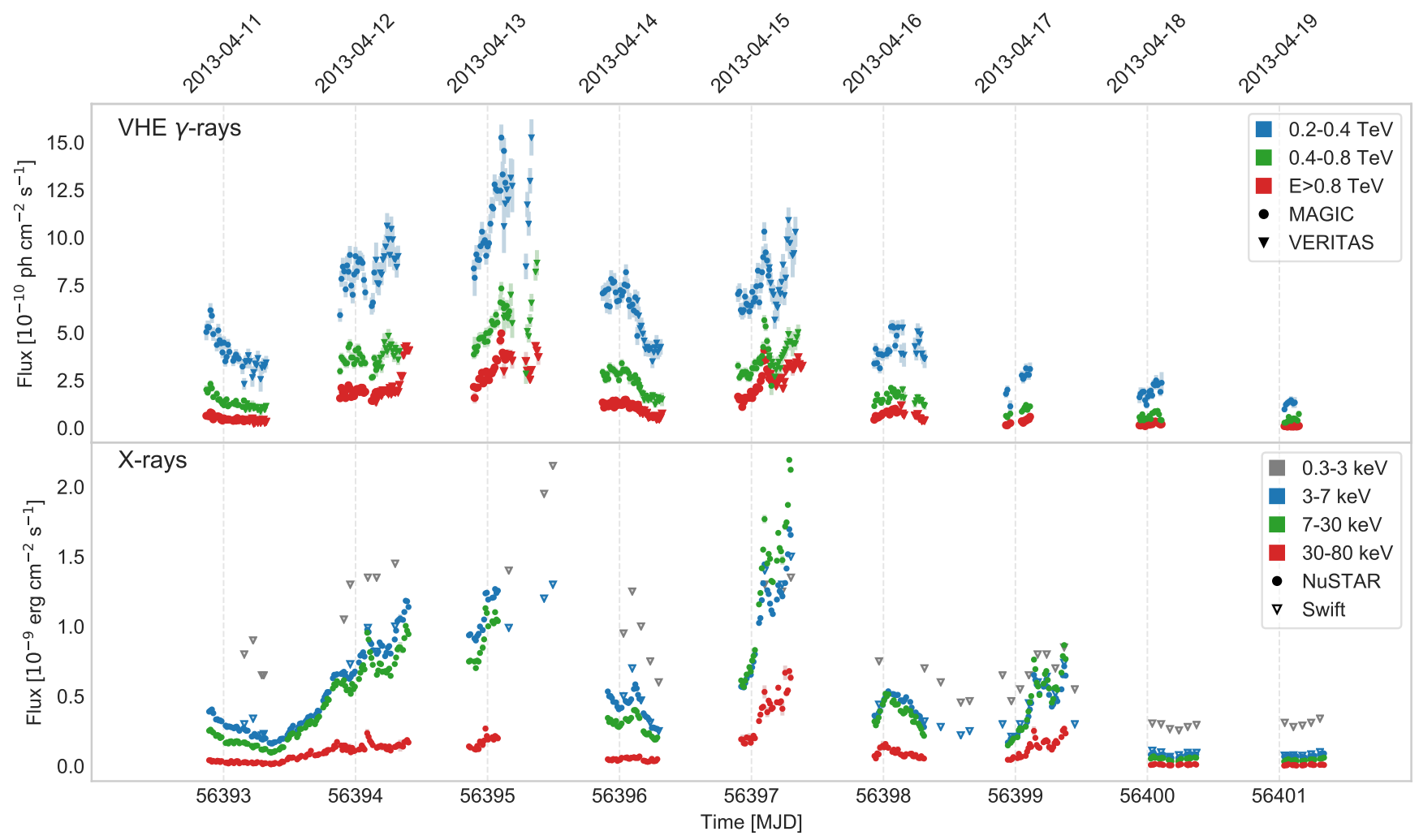

Figure 2. Light curves in various VHE and X-ray energy bands obtained with data from MAGIC, VERITAS and NuSTAR (split in 15 minute time bins) and Swift-XRT (from several observations with an average duration of about 17 minutes). For the sake of clarity, the $0.3-3 \mathrm{keV}$ fluxes have been scaled with a factor 0.5 . The statistical uncertainties are in most cases smaller than the size of the marker used to depict the VHE and X-ray fluxes.

\section{MULTI-BAND \& MULTI-TIMESCALE VARIABILITY}

\subsection{Fractional variability}

The flux variability reported in the multi-band light curves can be quantified using the fractional variability parameter $F_{\text {var }}$, as prescribed in Vaughan et al. (2003):

$$
F_{\mathrm{var}}=\sqrt{\frac{S^{2}-<\sigma_{\mathrm{err}}^{2}>}{<F_{\gamma}>^{2}}}
$$

$\left\langle F_{\gamma}\right\rangle$ denotes the average photon flux, $S$ the standard deviation of the $N$ flux measurements and $\left\langle\sigma_{\text {err }}^{2}\right\rangle$ the mean squared error, all determined for a given instrument and energy band. The uncertainty on $F_{\text {var }}$ is calculated using the prescription from Poutanen et al. (2008), as described in Aleksić et al. (2015a). This formalism allows one to quantify the variability amplitude, with uncertainties dominated by the flux measurement errors, and the number of measurements performed. The systematic uncertainties on the absolute flux measure- ments ${ }^{5}$ do not directly add to the uncertainty in $F_{\text {var }}$. The caveats in the usage of $F_{\mathrm{var}}$ to quantify the variability in the flux measurements performed with different instruments are described in Aleksić et al. (2014, 2015a,b). The most important caveat is that the ability to quantify the variability depends on the temporal coverage (observing sampling) and the sensitivity of the instruments used, which is somewhat different across the electromagnetic spectrum. A big advantage of the study presented here is that the temporal coverage for three bands in X-rays (from NuSTAR) and three bands in VHE gamma rays (from MAGIC and VERITAS) is exactly the same, which allows us to make a more direct comparison of the variability in these energy bands.

The fractional variability parameter $F_{\text {var }}$ was computed using the flux values and uncertainties reported in the light curves from Section 3 (see Fig. 1 and Fig. 2), hence providing a quantification of variability amplitude

\footnotetext{
5 The systematic uncertainties in the flux measurements at the radio, optical, X-ray and $\mathrm{GeV}$ bands are of the order of $10-15 \%$, while for the VHE bands are $\sim 20-25 \%$.
} 
for this nine-day long flaring activity from radio to VHE gamma-ray energies. The results are depicted in the upper panel of Fig. 3, where open markers are used for the variability computed with all the available data, and the filled markers are used for simultaneous observations. Given the slightly different temporal coverage for different VHE bands, as described in the previous section, we decided to use the $0.2-0.4 \mathrm{TeV}$ band to define the time slots for simultaneous X-ray/VHE observations. This ensures that the same temporal bins are being used for the $3 \times 3 \mathrm{X}$-ray and VHE bands. For comparison purposes, we added the $F_{\text {var }}$ values obtained for the period January to March 2013, when Mrk 421 showed a very low activity (see Baloković et al. 2016).

The fractional variability plot shows the typical double-bump structure, which is anlogous to the broadband SED. This plot shows that most of the flux variations occur in the X-ray and VHE bands, which correspond to the falling segments of the SED. Additionally, it also shows that, during the nine-day flaring activity in April 2013, the amplitude variability in the hard Xray band was substantially larger than that measured during the low activity from January to March 2013. The higher the X-ray energy, the larger the difference between the $F_{\text {var }}$ values from the low and the high activity.

In addition to the study of nine-day behavior, the high photon fluxes and the deep exposures allow us to compute the $F_{\text {var }}$ with the single-night light curves from six consecutive nights (from April 11 to April 17) ${ }^{6}$, hence allowing us to study the fractional variability on hour timescales for three X-ray bands and three VHE gammaray bands. For this study, only simultaneous data (using the time bins from the $0.2-0.4 \mathrm{TeV}$ band) were used, which means that the $3 \times 3 \mathrm{X}$-ray/VHE bands sample exactly the same source activity. The results are depicted in the bottom panels of Fig. 3. In general, all $F_{\text {var }}$ values computed with the single-night light curves are lower than those derived with the 9-day light curve for the corresponding energy band. This is clearly visible when comparing the data points with the grey shadowed regions in the upper panel of Fig. 3. Despite the X-ray and VHE flux varying on sub-hour timescales, the resulting intra-night fractional variability is significantly lower than the overall fractional variability in the nine-day time interval. This result is expected because, while for single days the light curves show flux variations within a factor of about 2 , the nine-day light

\footnotetext{
6 The light curves from April 18 and April 19 contain little data $(\sim 2$ hours) and little variability, which prevents the calculation of significant ( $>3$ sigma) variability for most of the energy bands.
}

curve shows flux variations larger than a factor of about 10. Unexpectedly, we find a large diversity in the variability versus energy patterns observed for the different days. For the days April 13, 15, 16, 17, one finds the typical pattern of higher fractional variability at higher photon energy within each of the two SED bumps. On the other hand, one finds that the fractional variability is approximately constant with energy for the days April 11 and April 14, and that the fractional variability decreases with energy for April 12. The decrease in $F_{\text {var }}$ with increasing energy is only marginally significant in the VHE bands $(\sim 2 \sigma)$, but very prominent in the X-ray emission, which within the synchrotron selfCompton (SSC) scenario, provides a direct mapping to the energy of the radiating electrons. These different variability versus energy patterns suggest the existence of diverse causes (or regions) responsible for the variability in the broadband blazar emission on timescales as short as days and hours. This is the first time that the variability of Mrk 421 can be studied with this level of detail, and the implications will be discussed in Section 6.

\subsection{Flux variations on multi-hours and sub-hours timescales}

This section focuses on the flux variations observed in the hard X-ray and VHE gamma-ray bands, which are the ones with the largest temporal coverage and highest variability (see section 4.1). The light curves for all nights for these $3 \times 3$ energy bands are reported in Appendix $B$. There is clear intra-night variability in all the light curves, which can be significantly detected because of the high fluxes and the good temporal coverage, as described in the previous section (e.g. see Fig. 3). The single-night light curves show a large diversity of temporal structures that relate to different timescales, from sub-hours (i.e. fast variation) to multi-hours (trends). We note that some of these fast components are present in both X-rays and VHE gamma rays, while some others are visible only at X-rays, or only at VHE gamma rays, and, in some cases, the features are present only in specific bands (either X-rays or VHE gamma rays) and not in the others. As it occurred with the study of the $F_{v a r}$ vs energy, the evaluation of the single-night multi-band light curves also suggests that there are different mechanisms responsible for the variability, some of them being achromatic (affecting all energies in similar way) and others chromatic (affecting the different energy bands in substantially different manner).

In this section we attempt to quantify the main trends and fast features, as well as their evolution across the various energy bands. We do that by fitting with a func- 

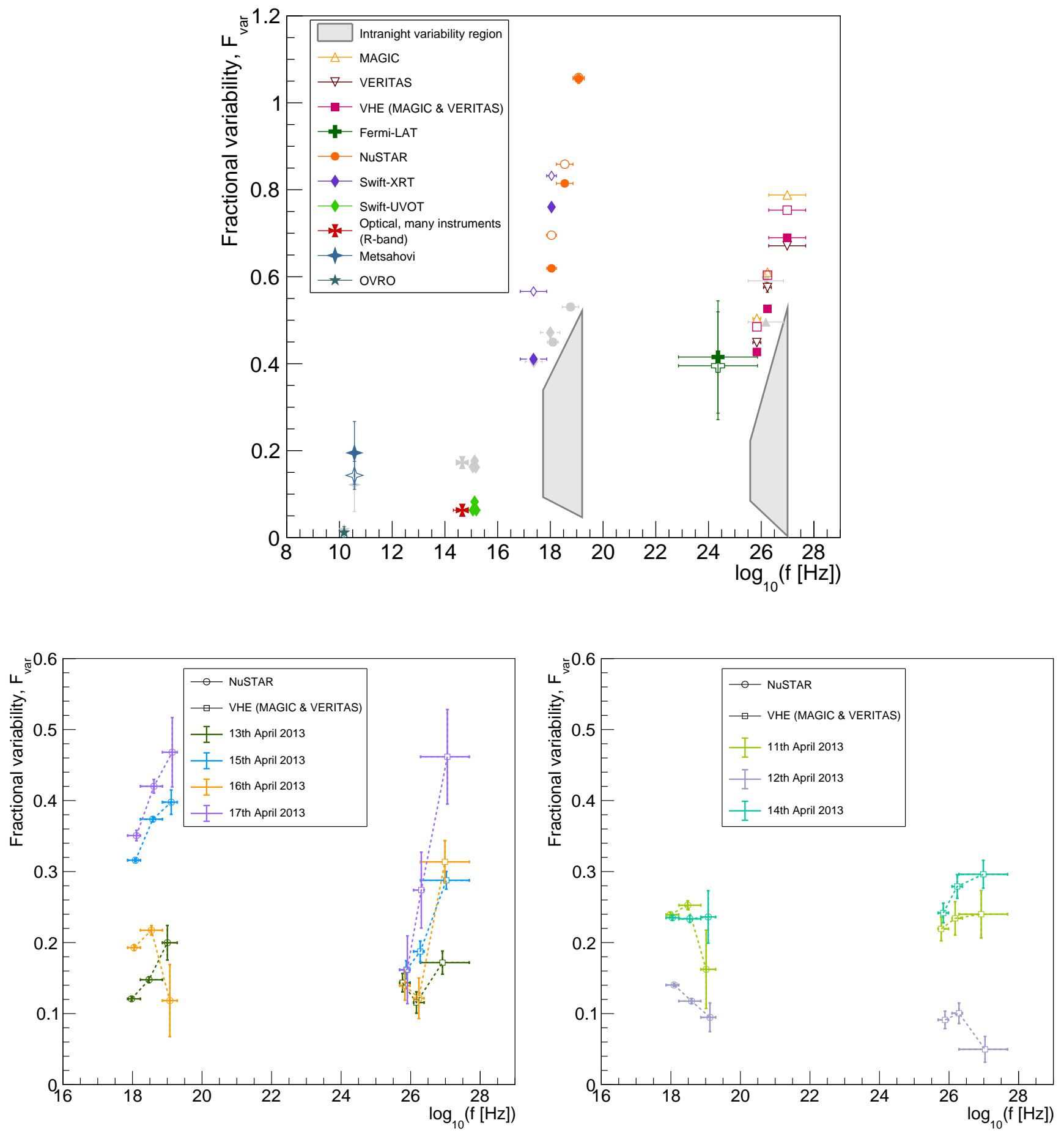

Figure 3. Upper panel: Fractional variability $F_{\text {var }}$ vs. energy band for the 9-day interval April 11-19. The panel reports the variability obtained using all available data (open symbols), and using only data which are taken simultaneously (filled symbols). For comparison, the $F_{\text {var }}$ vs. energy obtained with data from January-March 2013 (see Baloković et al. 2016) are also depicted with grey markers. The grey shadowed regions depict the range of $F_{\text {var }}$ obtained with data from single-night light

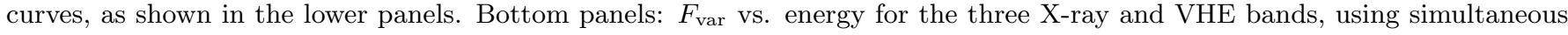
observations, and calculated for each night separately. In all plots, the vertical error bars depict the $1 \sigma$ uncertainty, while the horizontal error bars indicate the energy range covered. In order to improve the visibility of the data points, some markers have been slightly shifted horizontally (but always well within the horizontal bar). 
tion formed by a slow trend $F_{s}(t)$ and fast feature $F_{f}(t)$ components.

$$
F(t)=F_{s}(t)+F_{f}(t)
$$

where

$$
F_{s}(t)=\text { Offset } \cdot(1+\text { Slope } \cdot t)
$$

and

$$
F_{f}(t)=\frac{2}{2^{-\frac{t-t_{0}}{t_{\text {rise }}}}+2^{\frac{t-t_{0}}{t_{\text {fall }}}}} \cdot A \cdot F_{s}\left(t_{0}\right)
$$

Here $A$ is the flare amplitude, $t$ is the time since midnight for the chosen night, $t_{0}$ is the time of the peak flux of the flare, and $t_{\text {rise }}$ and $t_{\text {fall }}$ are the flux-doubling timescales for the rising and falling part of the flare. This formulation, with the slope of the slow component normalized to the offset, and the flare amplitude (of the fast component) normalized to the slow component at $t_{0}$, enables a direct comparison of the parameter values among the different energy bands, for which the overall measured flux may differ by factors of a few.

In general, we find that, whenever fast flares occur, they appear to be quite symmetric and, given the relatively short duration (sub-hour timescales) and the flux measurement uncertainties, we do not have the ability to distinguish (in a statistically meaningful way) between different rise- and fall-doubling times. For the sake of simplicity, we decided to fit the light curves with a function given by Eq. 5 where $t_{\text {rise }}=t_{\text {fall }}=$ flux-doubling time. This fit function provides a fair representation of the intra-night rapid flux variations from all days but for April 16, where the flux variations have much longer (multi-hour) timescales.

This relatively simple function provides a rough description of the energy-dependent light curves, and may not describe perfectly well all the data points. For instance, in the low energy X-ray bands, the statistical uncertainties are very small and one can appreciate significant and complex substructure that is not reproduced by the above-described (and relatively simple) fitting function. We do not intend to find a model that describes accurately all the data points. Rather we look for a model that provides a description of the main fluxvariability trends, and how they evolve with the X-ray and VHE energies.

The multi-band flux variations during April 15 and its related quantification using Eq. 3 are depicted in Fig. 4, with the parameters resulting from the fits reported in Table 3. The main multi-band emission varies on timescales of several hours, and hence it is dominated by the "slow component" in equation 3. The
Slope of this variation (quantified relative to the Offset in each band for better comparison among all bands) has a strong energy dependence, with the parameter value for the highest energies being around a factor of 2-3 times larger than that for the lowest energies for both the X-ray and VHE gamma-ray bands (e.g. Slope $_{>0.8 \mathrm{TeV}} \simeq 3 \cdot$ Slope $_{0.2-0.4 \mathrm{TeV}}$ ). The second most important feature of this multi-band light curve is the existence of a short flare, on the top of the slowly varying flux, in all the energy bands for both X-ray and VHE gamma rays. The location of the flare $t_{0}$ is the same (within uncertainties) in all the three X-ray bands and VHE bands. In order to quantify better the location of the short flare at X-ray and VHE gammaray energies, using the information from Table 3, we computed the weighted average separately for the three VHE bands, $t_{0, \mathrm{VHE}}=2.44 \pm 0.03 \mathrm{hr}$ and the three X-ray bands, $t_{0, \mathrm{X} \text {-ray }}=2.41 \pm 0.04 \mathrm{hr}$ past midnight. This indicates that, for this fast feature in the light curve, for all the energies probed, there is no delay in between $\mathrm{X}$-ray and VHE gamma-ray emission down to the resolution of the measurement, which, adding the errors in quadrature, corresponds to 3 minutes. The flux-doubling time is comparable among all the energy bands, with about 0.3 hours for all the X-ray bands and the highest VHE band $(>0.8 \mathrm{TeV})$, and about 0.2 hours for the lowest and middle VHE band. The characteristic by which the fast X-ray flare differs from the fast VHE flare is in the normalized flare amplitude $A$ (see Table 3 ): it is energyindependent (achromatic) for the X-ray fast flare, while it increases its value (chromatic) for the VHE fast flare (with amplitude $A_{>0.8 \mathrm{TeV}} \simeq 2 \cdot A_{0.2-0.4 \mathrm{TeV}}$ ).

In order to evaluate potential spectral variability throughout the $\sim 10$-hour light curves measured on 2013 April 15, we computed the flux hardness ratios $H R$ $\left(=F_{\text {high-energy }} / F_{\text {low-energy }}\right)$ for several energy bands in both X-ray and VHE gamma-ray domains. Fig. 5 depicts the $H R$ computed with the data flux measurements (in time bins of 15 minutes) and the $H R$ expected from the fitted functions reported in Fig. 4 and Table 3. For comparison purposes, we also included the $H R$ from the fitted functions from Table 3 excluding the fast component given by Eq. 5 (dotted line in Fig. 5). The dashed vertical red line indicates the weighted average time of the peak of the flare $t_{0}$, calculated separately for the three X-ray bands and VHE gammaray bands (see above). One can see that the overall impact of the fast component in the $H R$ temporal evolution is small, and only noticeable in some panels (e.g. $F_{>0.8 \mathrm{TeV}} / F_{0.4-0.2 \mathrm{TeV}}$ or $\left.F_{7-30 \mathrm{keV}} / F_{3-7 \mathrm{keV}}\right)$. This is due to the relatively short duration of the fast component, and the relatively small magnitude of the flare 

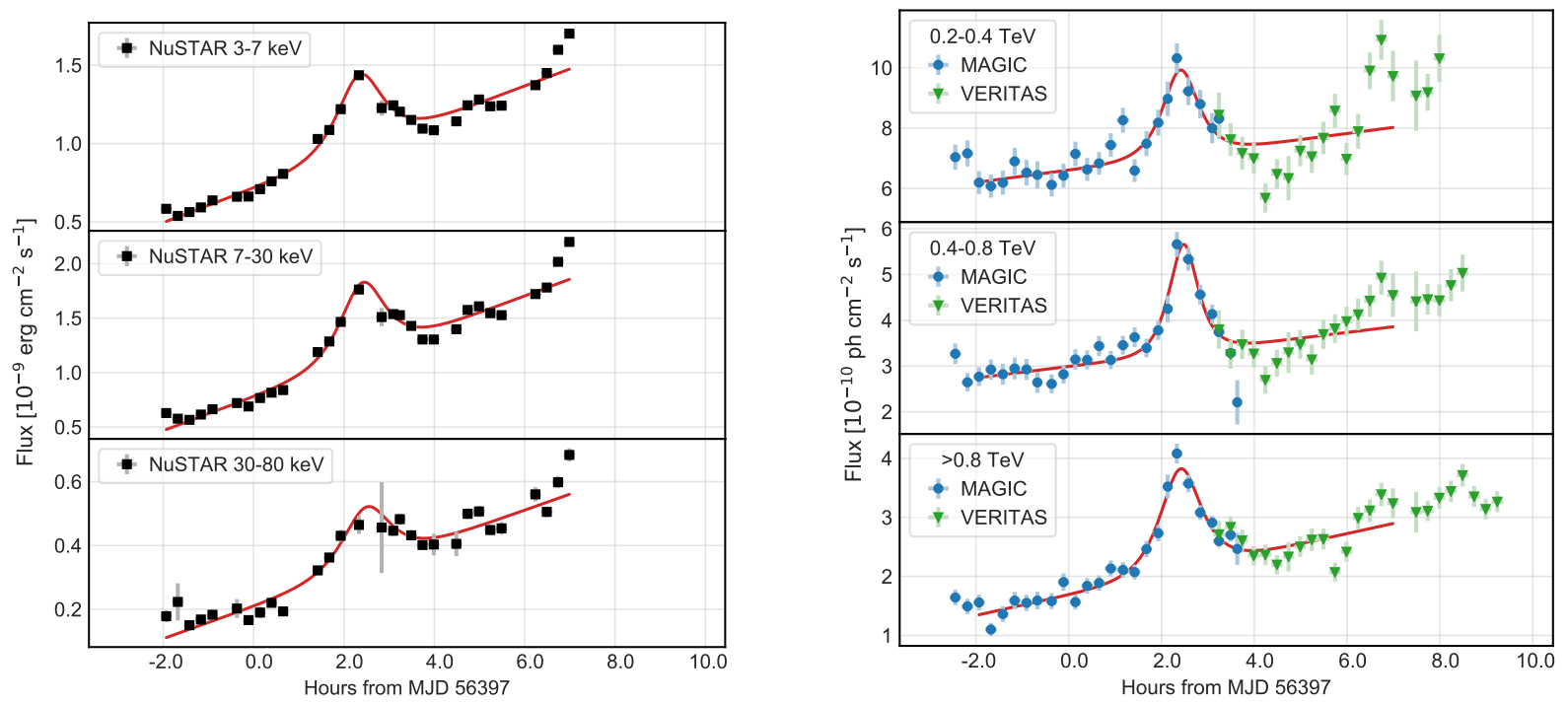

Figure 4. Light curves from 2013 April 15 in three X-ray bands (left panel) and three VHE gamma-ray bands (right panel). The red curve is the result of a fit with the function in Eq. 3, applied to the time interval with simultaneous X-ray and VHE observations. The resulting model parameters from the fit are reported in Table 3.

Table 3. Parameters resulting from the fit with Eq. 3 to the X-ray and VHE multi-band light curves from 2013 April 15.

\begin{tabular}{c|c|c|c|c|c|c}
\hline \hline Band & Offset $^{\mathrm{a}}$ & $\begin{array}{c}\text { Slope } \\
{\left[\mathrm{h}^{-1}\right]}\end{array}$ & $\begin{array}{c}\text { Flare } \\
\text { Amplitude } A\end{array}$ & $\begin{array}{c}\text { Flare } \\
\text { flux-doubling time }{ }^{\mathrm{b}}[\mathrm{h}]\end{array}$ & $\begin{array}{c}\text { Flare } \\
t_{0}[\mathrm{~h}]\end{array}$ & $\chi^{2} /$ d.o.f \\
\hline 15 April 2013 & \multicolumn{7}{l}{} \\
\hline $3-7 \mathrm{keV}$ & $0.71 \pm 0.01$ & $0.153 \pm 0.006$ & $0.49 \pm 0.07$ & $0.30 \pm 0.04$ & $2.35 \pm 0.06$ & $836 / 24$ \\
$7-30 \mathrm{keV}$ & $0.78 \pm 0.02$ & $0.199 \pm 0.009$ & $0.59 \pm 0.11$ & $0.30 \pm 0.04$ & $2.41 \pm 0.06$ & $889 / 24$ \\
$30-80 \mathrm{keV}$ & $0.21 \pm 0.01$ & $0.241 \pm 0.018$ & $0.56 \pm 0.18$ & $0.32 \pm 0.09$ & $2.50 \pm 0.10$ & $111 / 24$ \\
$0.2-0.4 \mathrm{TeV}$ & $6.60 \pm 0.17$ & $0.031 \pm 0.008$ & $0.40 \pm 0.09$ & $0.23 \pm 0.07$ & $2.41 \pm 0.09$ & $96.9 / 38$ \\
$0.4-0.8 \mathrm{TeV}$ & $2.99 \pm 0.07$ & $0.042 \pm 0.008$ & $0.72 \pm 0.09$ & $0.19 \pm 0.03$ & $2.47 \pm 0.04$ & $68.1 / 42$ \\
$>0.8 \mathrm{TeV}$ & $1.68 \pm 0.05$ & $0.103 \pm 0.010$ & $0.82 \pm 0.08$ & $0.27 \pm 0.03$ & $2.41 \pm 0.04$ & $90.0 / 45$ \\
\hline
\end{tabular}

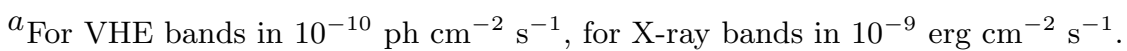

${ }^{b}$ Parameters $t_{\text {rise }}$ and $t_{\text {fall }}$ in Eq. 3 are set to be equal, and correspond to the Flare flux-doubling time in the Table.

amplitude, in comparison to the overall flux. Therefore, the temporal evolution of the spectral shape in both bands, X-ray and VHE, is dominated by the slow component, i.e. by the variations with timescales of several hours.

Besides April 15, we also performed the fit with Eq. 3 to the other five consecutive nights with large X-ray and VHE gamma-ray simultaneous datasets, namely all nights from April 11 to April 16 (both included). The results from these fits are reported in Appendix C (see Table 7 and Fig. 13-17). It is worth stating that, when comparing the quantification of the various light curves with the function Eq. 3, we found diversity among the fit parameter values and their energy dependencies. For April 11, we did not find any fast component, and the flux decreases monotonically through the observation with energy-independent Slope for both X-ray and VHE gamma rays (fully achromatic flux variations). On the other hand, during April 12, the emission increased throughout the observation, but with a Slope that decreases with increasing energy in both X-ray and VHE gamma rays. This trend is also observed, from a dif- 

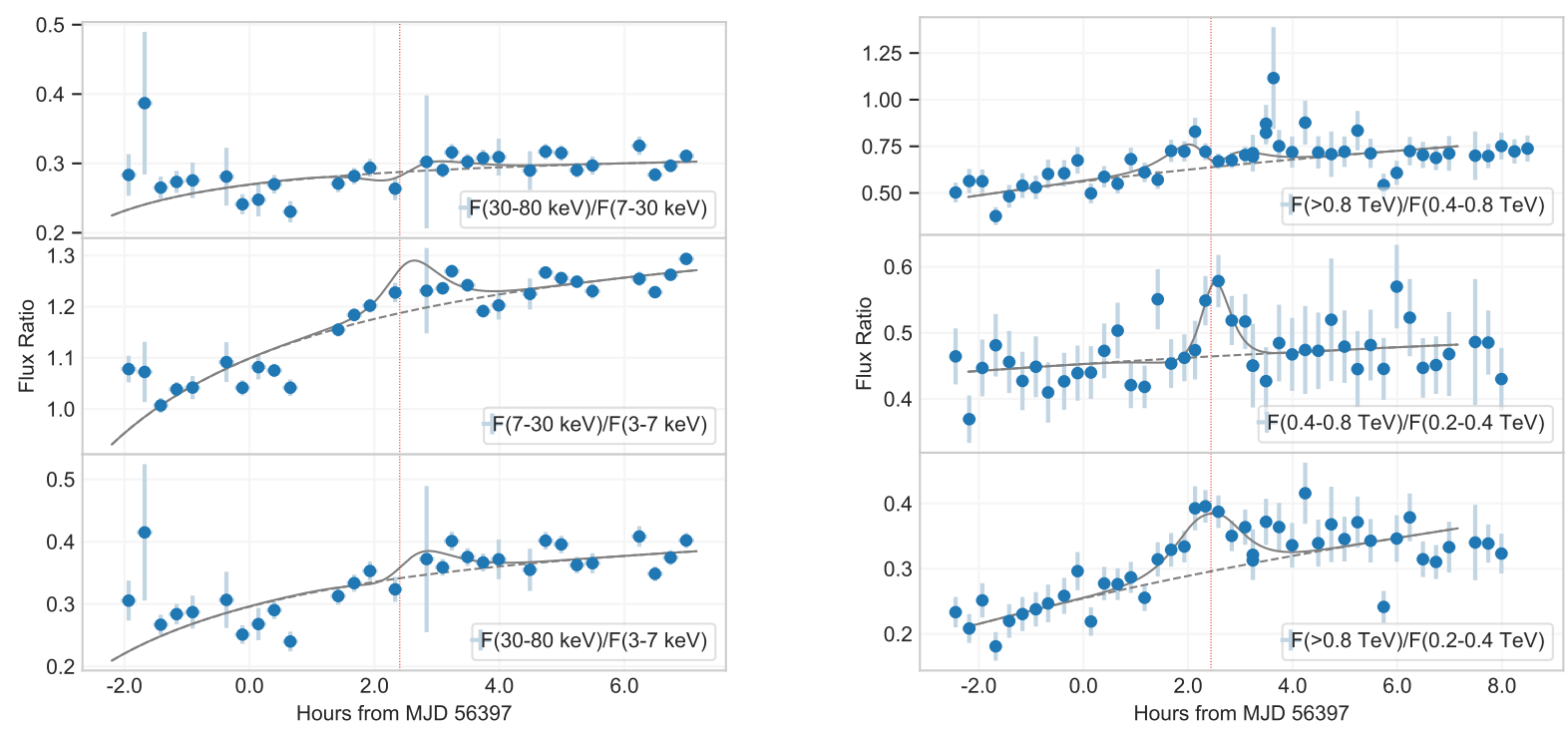

Figure 5. The X-ray hardness (flux) ratios for several X-ray (NuSTAR) bands (left panel) and VHE (MAGIC+VERITAS) bands (right panel) for April 15. In both panels, the dashed red vertical line indicates the average time of the peak of the flare in VHE $t_{0, \mathrm{VHE}}=2.44 \pm 0.03 \mathrm{hr}$, and X-rays $t_{0, \mathrm{X} \text {-ray }}=2.41 \pm 0.04 \mathrm{hr}$, where the average is calculated for the three bands (Table 3 ). The solid grey curve is the ratio of the fitted functions with parameters reported in Table 3, and the dashed grey line is the ratio of the same fitted functions, but this time excluding the fast component.

ferent perspective, in the bottom-right panel of Fig. 3, which displays a decreasing $F_{v a r}$ with increasing energy for both the X-ray and VHE gamma-ray emission from April 12. This is a very interesting behavior because it is opposite to the trend reported in most datasets from Mrk 421, where the variability increases with energy. For this night, we can also see a fast X-ray flare (flux-doubling time of about 0.3 hours) whose amplitude increases with energy. Unfortunately, this fast Xray flare occurred during a time window without VHE observations.

For April 13, we also observed a slow flux variation with an energy-independent Slope, as in April 11, but this time with a flux increase, instead of a decrease. Additionally, we did observe a super-fast X-ray flare (fluxdoubling time of $5 \pm 1$ minutes) without any counterpart in the VHE light curve, i.e. an 'orphan' X-ray flare (see Fig. 15). As shown in Table 7, the X-ray NusTAR flare amplitude relative to the overall baseline is only about $11 \%$, but it is significant (3-4 $\sigma$ depending on the energy band) and there is no correlated flux variation in the simultaneous VHE MAGIC fluxes, which have flux uncertainties of about $5 \%$.

During April 14th we see again a monotonically decreasing flux with an energy-independent Slope for both $\mathrm{X}$-ray and VHE gamma rays, with another fast X-ray flare (flux-doubling time $\sim 0.5$ hours) without counterpart in the VHE light curve.
The night that differs most is April 16, which does not show any monotonic increase or decrease, and a largely non-symmetric flare with flux variation timescales of hours. In order to quantify the temporal multi-band evolution of the flux during April 16, we used Eq. 5 (i.e. the fitting function without the slow component), with $t_{\text {rise }} \neq t_{\text {fall }}$. See Appendix C for further details about the quantification of the multi-band flux variations during the six consecutive nights, from April 11 to April 16.

In summary, during these six consecutive nights with enhanced activity and with multi-hour long X-ray/VHE simultaneous exposures in April 2013, we found achromatic and chromatic flux variability with timescales spanning from multi-hours to sub-hours, and several Xray fast flares without VHE gamma-ray counterparts. We did not see any VHE gamma-ray orphan fast flare (whenever we had simultaneous X-ray coverage). However, we did observe fast flares in some specific energy bands which are not detected in the other nearby energy bands (X-ray or VHE); which suggests the presence of flaring mechanisms affecting relatively narrow energy bands.

The temporal evolution of the X-ray and VHE emission, and the particularity of being able to approximately describe it with a two-component function with a fast (sub-hour variability timescale) and slow (multihour variability), will be discussed in section 6 . 
Table 4. Correlation coefficients and slopes of the linear fit to the VHE vs. X-ray flux (in log scale) derived with the 9-day flaring episode of Mrk 421 in April 2013.

\begin{tabular}{|c|c|c|c|c|c|c|}
\hline VHE band & X-ray band & Pearson coeff. ${ }^{a}$ & $N \sigma$ Pearson $^{a}$ & $\mathrm{DCF}$ & Linear fit slope & $\chi^{2} /$ d.o.f \\
\hline \multirow[t]{3}{*}{$0.2-0.4 \mathrm{TeV}$} & $3-7 \mathrm{keV}$ & $0.92 \pm 0.01$ & 20.2 & $0.93 \pm 0.12$ & $0.61 \pm 0.02$ & $1183 / 162$ \\
\hline & $7-30 \mathrm{keV}$ & $0.87 \pm 0.02$ & 17.0 & $0.88 \pm 0.11$ & $0.45 \pm 0.03$ & $1891 / 162$ \\
\hline & $30-80 \mathrm{keV}$ & $0.79 \pm 0.03$ & 13.6 & $0.81 \pm 0.11$ & $0.35 \pm 0.02$ & $2277 / 162$ \\
\hline \multirow[t]{3}{*}{$0.4-0.8 \mathrm{TeV}$} & $3-7 \mathrm{keV}$ & $0.946_{-0.009}^{+0.007}$ & 23.4 & $0.96 \pm 0.11$ & $0.79 \pm 0.03$ & $1038 / 170$ \\
\hline & $7-30 \mathrm{keV}$ & $0.91 \pm 0.01$ & 19.8 & $0.92 \pm 0.11$ & $0.58 \pm 0.03$ & $1725 / 170$ \\
\hline & $30-80 \mathrm{keV}$ & $0.84 \pm 0.02$ & 15.8 & $0.86 \pm 0.11$ & $0.45 \pm 0.03$ & $2160 / 170$ \\
\hline \multirow[t]{3}{*}{$>0.8 \mathrm{TeV}$} & $3-7 \mathrm{keV}$ & $0.964_{-0.006}^{+0.005}$ & 26.0 & $0.97 \pm 0.11$ & $1.11 \pm 0.03$ & $704 / 170$ \\
\hline & $7-30 \mathrm{keV}$ & $0.947_{-0.008}^{+0.007}$ & 23.5 & $0.96 \pm 0.11$ & $0.81 \pm 0.03$ & $1245 / 170$ \\
\hline & $30-80 \mathrm{keV}$ & $0.89 \pm 0.02$ & 18.6 & $0.91 \pm 0.10$ & $0.61 \pm 0.03$ & $1736 / 170$ \\
\hline
\end{tabular}

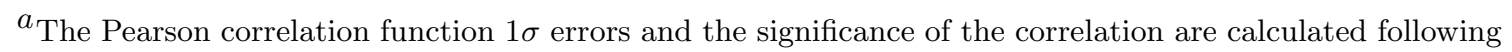
Press et al. (2002).

\section{UNPRECEDENTED STUDY OF THE MULTI-BAND X-RAY AND VHE GAMMA-RAY CORRELATIONS}

We evaluated the correlations among all the frequencies covered during the April 2013 flare, and found that the largest flux variations and the largest degree of flux correlation occurs in the X-ray and VHE gammaray bands. No correlation was found among the radio, optical and gamma-ray bands, a result that was expected because of the lower activity and longer variability timescales at these energies. Apart from some variability in the GeV flux around April 15, which is the day with the highest X-ray activity, the $\mathrm{GeV}$ emission appears constant for the 12-hour time intervals related to flux variations by factors of a few at $\mathrm{keV}$ and $\mathrm{TeV}$ energies. If the $\mathrm{GeV}$ and $\mathrm{TeV}$ fluxes were correlated on 12-hour time scales, Fermi-LAT should have detected large flux variations, and hence we can exclude this correlation.

The quality and extent of this dataset, both in time and energy, allows for a X-ray/VHE correlation study that is unprecedented among all datasets collected from Mrk 421, and any other TeV blazar. The relation between the VHE gamma-ray and the X-ray fluxes in the $3 \times 3$ energy bands is shown in Fig. 6 for the 9-day flaring activity, and in Fig. 7 for April 15th. The Discrete Correlation Function (DCF) and Pearson correlation coefficients, as well as the slope of the VHE versus X-ray flux are reported in Table 4. There is a clear pattern: the strength of the correlation increases for higher VHE bands and lower X-ray bands. The strongest correlation is observed between the $3-7 \mathrm{keV}$ and $>0.8 \mathrm{TeV}$ bands. This combination of bands also shows a slope (from the fit in Table 4) closest to 1 , among all the $3 \times 3$ bands reported. Moreover, the scatter in the plots is smaller as we increase the VHE band and decrease the X-ray energy band. The smallest scatter, which can be quantified with the $\chi^{2}$ of the fit (lower values of $\chi^{2}$ relate to smaller scatter in the data points), occurs for the combination $>0.8 \mathrm{TeV}$ and $3-7 \mathrm{keV}$.

Fig. 6 reveals that the different days occupy (roughly) different regions in the VHE versus X-ray flux plots (for all the $3 \times 3$ bands). This is expected because the largest flux changes occur on day-long timescales. In addition, individual days appear to show different patterns. In order to better characterize these different patterns (observed for the different days), we also computed the same quantities (DCF, Pearson and linear fit) to the simultaneous data points from the single nights with multi-hour light curves (namely April 11-16). The results are reported in Table 9, in Appendix D.

The main conclusions from this study performed on data from April 11 to April 16 are the following ${ }^{7}$ :

- In some nights, namely on April 15 and April 16, Mrk 421 shows the "general trend" that is observed for the full 9-day flaring activity, with the highest magnitude and significance in the correla-

\footnotetext{
${ }^{7}$ On the nights April 17-19, both the level of activity of Mrk 421 and the amount of data collected was substantially smaller, which prevents us from making detailed studies of the multi-band correlations.
} 


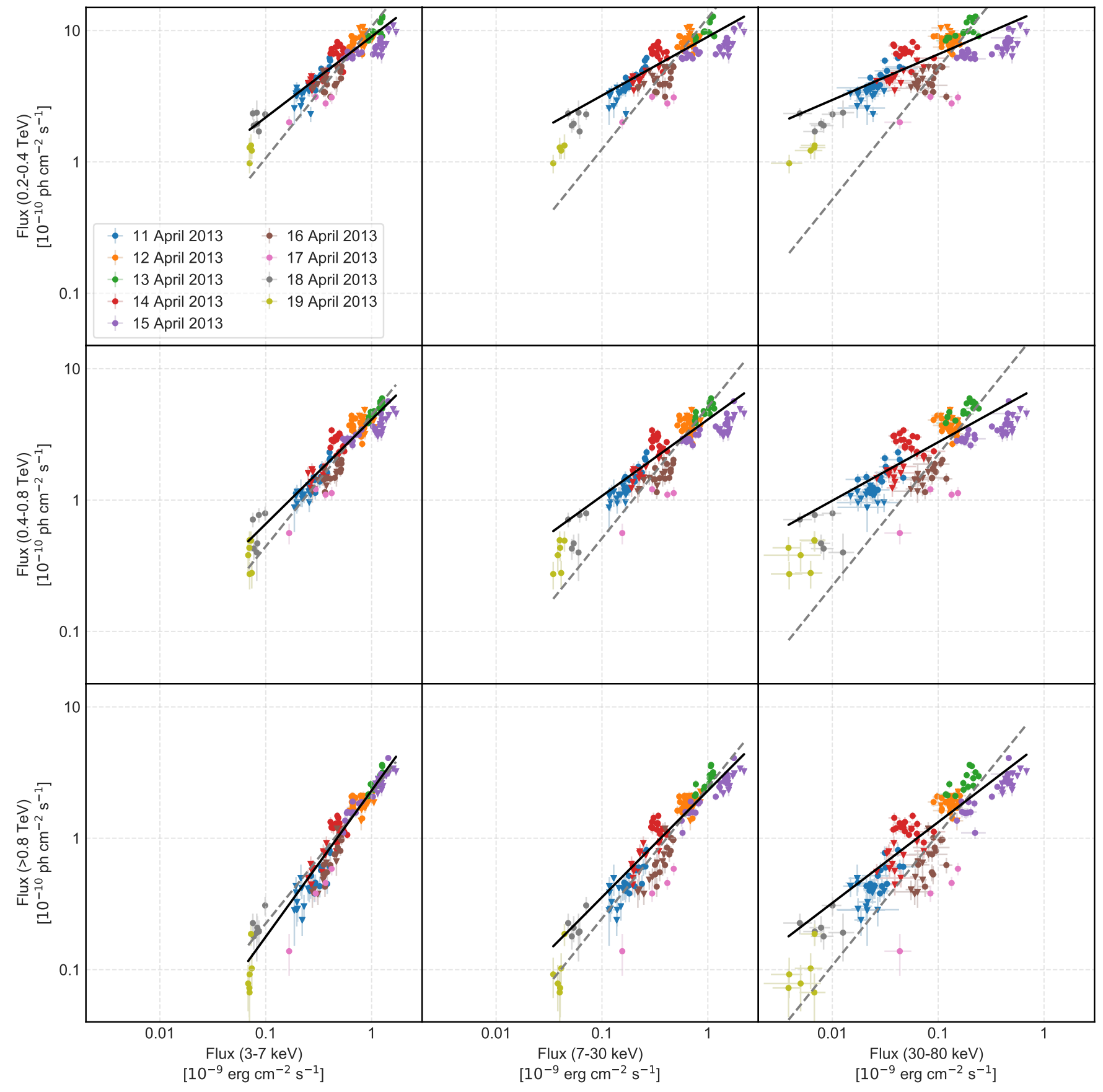

Figure 6. VHE flux vs. X-ray flux in three X-ray and three VHE energy bands. Data from all nine days is shown, with colors denoting fluxes from the different days. The grey dashed line is a fit with slope fixed to 1 , and black line is the best fit line to the data, with the slope quoted in Table 4. 


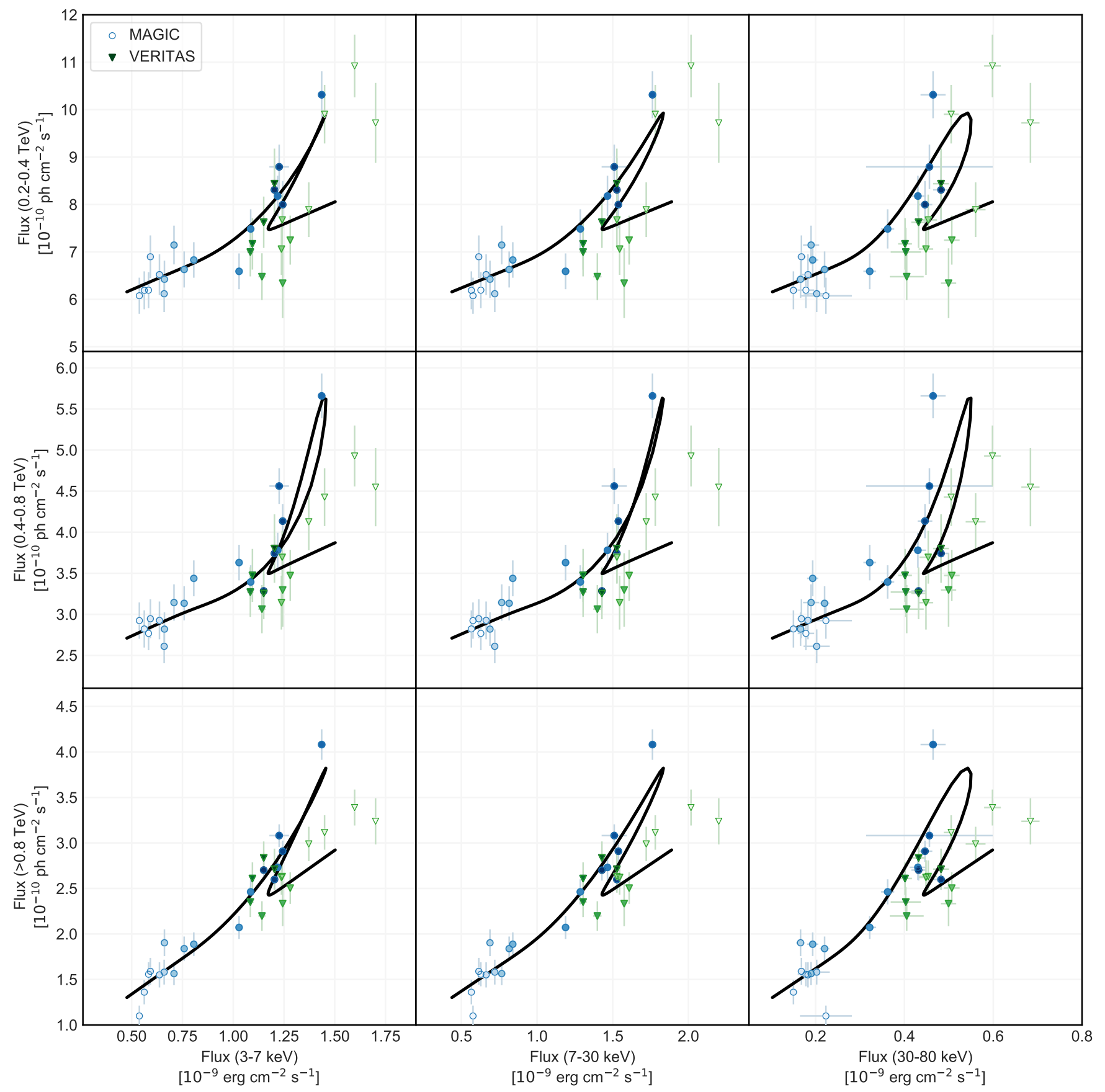

Figure 7. VHE flux vs. X-ray flux in three X-ray and three VHE energy bands for April 15 . The black line is the track predicted by Slow+Fast component fit from Eq. 3. The lightness of symbols follows time: for MAGIC data lightness decreases with time, and for VERITAS data it increases in time, so that the central part of the night, where MAGIC and VERITAS observations overlap, is plot using darker symbols. 
tion occurring for the $>0.8 \mathrm{TeV}$ versus the $3-7 \mathrm{keV}$ bands.

- In other nights, namely on April 11, 13-14, the general trend from the 9-day data set is less visible: there is a larger similarity in the magnitude and significance of the correlation among the various energy bands.

- In one night, April 12, we found no correlation between the X-ray and VHE gamma-ray bands, despite the significant variability in both bands. The lack of correlation between X-ray and VHE gamma rays (being both highly variable and characterized with simultaneous observations) has not been observed to date in Mrk 421, although it has been observed in another HBL (PKS 2155-304, in August-September 2008, Aharonian et al. 2009).

The correlation study was also performed splitting the data set into two subsets: (a) April 15, 16, and 17 (which appear somewhat away from the main trend in Fig. 6); and (b) April 11, 12, 13, 14, 18, and 19. In comparison to the nine-day data set, the two subsets analyzed separately yields a reduction of the scatter of the flux points around the main trends (which show up as a noticeable reduction in the $\chi^{2}$ values from the fit), and also a smaller dependence of the magnitude and significance of the correlations with the specific combination of VHE and X-ray energy bands. The largest linear fit slope occurs for the combination $>0.8 \mathrm{TeV}$ and $3-7 \mathrm{keV}$ in both subsets (as with the nine-day data set), but while for subset (a) we continue having the largest significance and magnitude of the correlation for $>0.8 \mathrm{TeV}$ and $3-7 \mathrm{keV}$, in subset (b) the change in magnitude and correlation with energy bands is much smaller, and the highest values occur for $>0.8 \mathrm{TeV}$ and $7-30 \mathrm{keV}$. This indicates a somewhat different physical state of the source during those three consecutive days (April 15,16 and 17) with respect to the others.

Besides the VHE gamma-ray and the X-ray fluxes in the $3 \times 3$ energy bands for April 15, Fig. 7 depicts also the flux-flux values from the fitted functions reported in Section 4.2. This figure shows that multiple components in the flux evolution (e.g. fast component on the top of the slow component) appear as "different trends" in the flux-flux plots with the flaring component having sharper VHE flux rise (with increasing X-ray flux) than the slow component. Because of the statistical uncertainties in the flux measurements, as well as the fact that one component has a much smaller flux and shorter duration, even for very good datasets such as this one, it is not easy to recognize and separate the contribution of different components in the flux-flux plots. However, these different patterns can produce collective deviations (when considering many of these different single-trends) that are statistically significant when fitting the data points in the flux-flux plots with simple trends, such as the linear or quadratic functions in the log-log scale.

The discussion of these observational results is given in Section 6.

\section{DISCUSSION OF THE RESULTS}

Although detailed spectral energy distribution and light curve modeling are beyond the scope of this paper, we discuss the main results of our analysis and provide possible interpretations.

\subsection{Minimum Doppler factor}

In general, VHE gamma rays can interact with low-energy synchrotron photons in order to produce electron-positron pairs. If both VHE gamma-ray and low-energy photons are produced in the same region, then the criterion that this attenuation is avoided so that the VHE gamma rays may escape from the source and be detected leads to a lower limit on the Doppler factor (Dondi \& Ghisellini 1995; Tavecchio et al. 1998; Finke et al. 2008). Owing to the detection of $10 \mathrm{TeV}$ photons from Mrk 421 during this flaring activity, the relevant observed synchrotron frequency for their attenuation is close to $6 \times 10^{12} \mathrm{~Hz}$. In the $R$ band $\left(\nu \sim 4.5 \times 10^{14}\right.$ $\mathrm{Hz}$ ), the observed flux is close to $50 \mathrm{mJy}$ on 2013 April 15. Using $12 \mathrm{~min}$ as the shortest variability timescale (see Table 3), and extrapolating the $R$ band flux to $6 \times 10^{12} \mathrm{~Hz}$ assuming the same spectral shape as the one obtained from the long-term SED (i.e., photon index $\sim 1.6$, Abdo et al. 2011), one finds $\delta \gtrsim 35$, which lies to the high-end of values derived from SED modeling of Fermi-LAT detected blazars (Ghisellini et al. 2010; Tavecchio et al. 2010; Paliya et al. 2017). The derived lower limit can be relaxed if the gamma-ray and optical emitting regions are decoupled, but the actual value would depend on the details of the theoretical model.

\subsection{Flux-flux correlations}

Flux-flux correlations have been the focus of many multi-wavelength campaigns during active and low states of blazar emission (for Mrk 421, see e.g. Maraschi et al. 1998; Fossati et al. 2008; Aleksić et al. 2015b), because their study may differentiate among emission models (see e.g. Krawczynski et al. 2002). Fossati et al. (2008), in particular, studied the correlations on a daily 
basis between $\mathrm{TeV}$ fluxes $(E>0.4 \mathrm{TeV})$ and $\mathrm{X}$-ray fluxes, mostly in the $2-10 \mathrm{keV}$ band, but also in the 2-4 keV, 9-15 keV and $20-60 \mathrm{keV}$ bands. The work we show here goes one step further, as it allows for the first time, to study flux-flux correlations between multiple VHE gamma-ray and X-ray energy bands on a daily basis and down to 15-min time bins.

The overall strong correlation found between the $>0.8 \mathrm{TeV}$ band and the lowest energy X-ray band (3-7 keV; Fig. 6 and Table 9) implies that the emissions are most likely co-spatial and they are produced by electrons with approximately the same energy via synchrotron in X-rays and SSC processes in gamma rays. In TeV blazars, like Mrk 421, the peak of the SSC spectrum is typically produced by inverse Compton scatterings in the Klein-Nishina regime (e.g., Tavecchio \& Ghisellini 2016).

The results presented in Section 5 reveal, however, a more complicated picture, as the strong correlation mentioned above weakens or even disappears on certain days (e.g., 2013 April 12). A weak correlation between $\mathrm{X}$-rays and gamma rays can be produced if the emissions are produced by different components (e.g. Petropoulou 2014; Chen et al. 2016) or by different particle populations, as in lepto-hadronic models (Mastichiadis et al. 2013). Different strengths of the correlation can be predicted by adjusting the temporal variations of the model parameters (e.g., injection rate of accelerated particles) and/or by having more than one emitting component in $\mathrm{X}$-rays and gamma rays.

The analysis presented in Section 5 also reveals that the slope of the correlation between the X-ray bands and the gamma-ray bands is generally sub-linear, i.e. $F_{\gamma} \propto F_{X}^{m}$ with $m \lesssim 1$, and changes with time (Table 9 ). In the standard (one-zone) SSC scenario, a value $m=2$ is expected if only the electron distribution normalization is varying with time. Even in this scenario though, different values of $m$ can be obtained when looking at correlations between different energy bands in X-rays and gamma rays (Katarzyński et al. 2005). Moreover, values $m<2$ are possible if several parameters changes with time (e.g., magnetic field strength and Doppler factor). Katarzyński et al. (2005) explored in detail these flux-flux correlations for an SSC model for high-peaked BL Lac objects like Mrk 421. They showed that $m \leq 1$ is expected between energy bands close to the peaks of the synchrotron and SSC components, if the blob is expanding and the magnetic field is decreasing (see Fig. 4 in Katarzyński et al. 2005). Petropoulou (2014) showed that in a two-component SSC scenario the slope of the correlation between $2-10 \mathrm{keV}$ and $0.4-10 \mathrm{TeV}$ may vary strongly from $m \sim 0$ to $\sim 1$ on day-long timescales, with a pattern that depends on the varying model parameter (i.e., injection rate or maximum electron energy). A quadratic relation between X-rays and gamma rays is also expected in lepto-hadronic models, where the former act as targets for the photo-hadronic interactions of accelerated protons that result in the production of gamma rays (Dimitrakoudis et al. 2012; Mastichiadis et al. 2013). A slope of $m \lesssim 1$ can also be produced in proton synchrotron models, where variations in the electron and proton injection rates are directly mapped to variations in the X-ray and gamma-ray flux, respectively (Mastichiadis et al. 2013). The detailed modeling of the flux-flux correlations will be the topic of a future study.

\subsection{Temporal variability}

One of the main results of this work is the detection of fast-evolving flares on top of a slower evolving emission in both X-ray and VHE gamma-ray bands. Although this temporal behavior was qualitatively discussed for some of the X-ray NuSTAR light curves in Paliya et al. (2015), here we present a quantitative study of these characteristics in the X-ray light curves and also the VHE gamma-ray light curves (see Fig. 4 in Section 4.2 and Figs. 13-17 in Appendix C). In the next paragraphs, we discuss possible interpretations for the origin of the multi-band temporal variability.

\subsubsection{Acceleration and cooling processes}

The rise and decay timescale of a flare may be associated with the acceleration and cooling timescales of the radiating electrons. In this case, one can use the fact that the acceleration and cooling timescales are found to be equal to estimate the magnetic field of the emitting region, as follows. If electrons undergo Fermi-1 (or Fermi-2) acceleration, then the acceleration timescale, in the co-moving frame, can be written as:

$$
t_{a c c}^{\prime}=\frac{2 \pi m_{e} c \gamma^{\prime} N_{a}}{e B^{\prime}}
$$

(e.g., Finke et al. 2008) where $B^{\prime}$ is the tangled (comoving) magnetic field strength, $\gamma^{\prime}$ is the electron Lorentz factor in the co-moving frame, and $N_{a} \geq 1$ is the number of gyrations an electron makes to double its energy. The synchrotron cooling timescale is

$$
t_{\mathrm{syn}}^{\prime}=\frac{6 \pi m_{e} c^{2}}{4 c \sigma_{\mathrm{T}} B^{\prime 2} \gamma^{\prime}} .
$$

Setting $t_{\mathrm{acc}}^{\prime}=t_{\mathrm{syn}}^{\prime}$ results in

$$
B^{\prime}=\frac{3 e}{4 \sigma_{\mathrm{T}} \gamma^{\prime 2} N_{a}} .
$$


The Lorentz factor of electrons producing the peak of the SSC mission can be estimated as:

$$
\gamma_{b}^{\prime} \approx \frac{E_{\mathrm{ssc}}}{\delta m_{e} c^{2}} \approx 5 \times 10^{4}\left(\frac{\delta}{40}\right)^{-1}\left(\frac{E_{\mathrm{ssc}}}{1 \mathrm{TeV}}\right)
$$

where $E_{\mathrm{ssc}}$ is the observed energy of the peak of the SSC component. Here we use a value of $\delta$ consistent with the lower limit from $\gamma \gamma$ pair production (Section 6.1). Here and from this point forward, we neglect factors of $1+z$ which will be quite small given the redshift of Mrk 421 $(z=0.03)$. To accelerate electrons to this peak requires

$$
B^{\prime} \approx 9\left(\frac{\delta}{40}\right)^{2}\left(\frac{N_{a}}{100}\right)^{-1} \mathrm{G}
$$

Large values of the magnetic field or large values of $N_{a}$ are required to accelerate electrons to $\gamma_{b}^{\prime}$ where $t_{\text {acc }}^{\prime}=$ $t_{\mathrm{syn}}^{\prime}$. Similar results were found for the luminous, rapid flare from PKS 2155-304 in 2006 (Finke et al. 2008).

Acceleration and cooling may control the light curve time scales, if $R_{b}^{\prime} / c \ll t_{\text {syn }}^{\prime}=t_{\text {acc }}^{\prime}$ or $R_{b}^{\prime} \ll 1.4 \times$ $10^{12}(\delta / 40)^{-3}\left(N_{a} / 100\right)^{-2} \mathrm{~cm}$, where $R_{b}^{\prime}$ is the radius of a spherical emitting region in the comoving frame. The required upper limit on $R_{b}^{\prime}$ is $2-3$ orders of magnitude smaller than typical values for the size of the emitting region (e.g., Abdo et al. 2011), even for flaring episodes (e.g., Aleksić et al. 2015b). Additionally, if the flare's rise and decay times were dominated by the acceleration and cooling timescales, one would expect the flaring timescales to be energy-dependent; however, they seem to have the same timescale across energy bands (see Table 3). Therefore, the timescales of the fast component of the light curve are likely controlled by the light-crossing time of a blob with fixed size. As a result, they should appear symmetric and with timescales independent of the energy.

\subsubsection{Plasmoids in magnetic reconnection}

Magnetic reconnection is invoked as an efficient particle acceleration process in a variety of astrophysical sources of non-thermal high-energy radiation including AGN jets (Romanova \& Lovelace 1992; Giannios et al. 2009, 2010; Giannios 2013). It has been proposed that plasmoids (i.e., blobs of magnetized plasma containing energetic particles) that are formed and accelerated in the reconnection regions of jets can serve as high-energy emission sites in both blazars and radio galaxies (Giannios et al. 2009; Sironi et al. 2015). Petropoulou et al. 2016, (hereafter, PGS16) presented a semi-analytic model of flares powered by plasmoids in a reconnection layer, simplifying the results of detailed particle-in-cell (PIC) simulations (for a full numerical treatment, see Christie et al. 2019).
A single plasmoid produces a flare with peak luminosity and flux-doubling timescale that depend on its size and Doppler beaming. A unique feature of this model is that the flux-doubling timescale in the rising part of the flare is mostly determined by the acceleration of the plasmoid in the layer (i.e., by its bulk motion). As a result, similar rise timescales should be observed at different energy bands of a flare powered by a single plasmoid (achromatic behavior). In contrast to the rising part of a flare, its decay is not constrained by PIC simulations. By setting the decay timescale to be approximately equal to the rise timescale of the fast flares, as observed, one can infer the declining rate at which accelerated particles are injected in the plasmoid or the decay rate of the magnetic field after the plasmoid has left the layer (PGS16).

No strong spectral evolution is expected during a flare produced by a single plasmoid (PGS16; Christie et al. 2019). At any given time though, an observer receives radiation from a large number of plasmoids in the layer, having different sizes and Doppler factors. Those plasmoids that move with mildly relativistic speeds (in the jet frame) and have intermediate sizes (in terms of layer's size) can contribute and even dominate the overall emission. The superposition of their emission could result in a slow varying and more luminous component of the light curve (Giannios 2013; Christie et al. 2019), which may exhibit spectral variations and drive the fractional variability on longer timescales than the fast varying component of the light curve (Christie et al. 2019b, in preparation).

Energetics \& timescales - PGS16 provided simple formulae to estimate the flux-doubling timescale $\left(t_{1 / 2}\right)$ and peak luminosity $\left(L_{\mathrm{pk}}\right)$ of flares produced by individual plasmoids in a reconnection layer (with half-length $L^{\prime 8}$ ) of a blazar jet (for an illustration, see Fig. 3 in Christie et al. 2019). The bolometric peak flare luminosity can be written as:

$$
L_{\mathrm{pk}, \mathrm{bol}}=\frac{\pi}{2} \beta_{\mathrm{g}} c w_{\mathrm{f}}^{\prime 2} \delta_{\mathrm{p}, \mathrm{f}}^{4} u_{\mathrm{e}}^{\prime}
$$

where $\beta_{\mathrm{g}}$ is the growth rate of a plasmoid, $w_{\mathrm{f}}^{\prime}$ and $\delta_{\mathrm{p}, \mathrm{f}}$ are, respectively, the plasmoid transverse size and Doppler factor at the end of its lifetime (i.e., when it is being advected from the layer or when it merges with a another bigger plasmoid). The Doppler factor takes into account the relativistic motion of the plasmoid in the layer and the relativistic motion of the layer itself (for definition, see Eq. 8 in PGS16). In the above equation, $u_{\mathrm{e}}^{\prime} \simeq f_{\text {rec }} L_{\mathrm{j}} / 4 \pi \varpi^{2} c \beta_{\mathrm{j}} \Gamma_{\mathrm{j}}^{2}$, where $\Gamma_{\mathrm{j}}$ is the jet's bulk

\footnotetext{
${ }^{8}$ Primed quantities are measured in the jet's rest frame.
} 


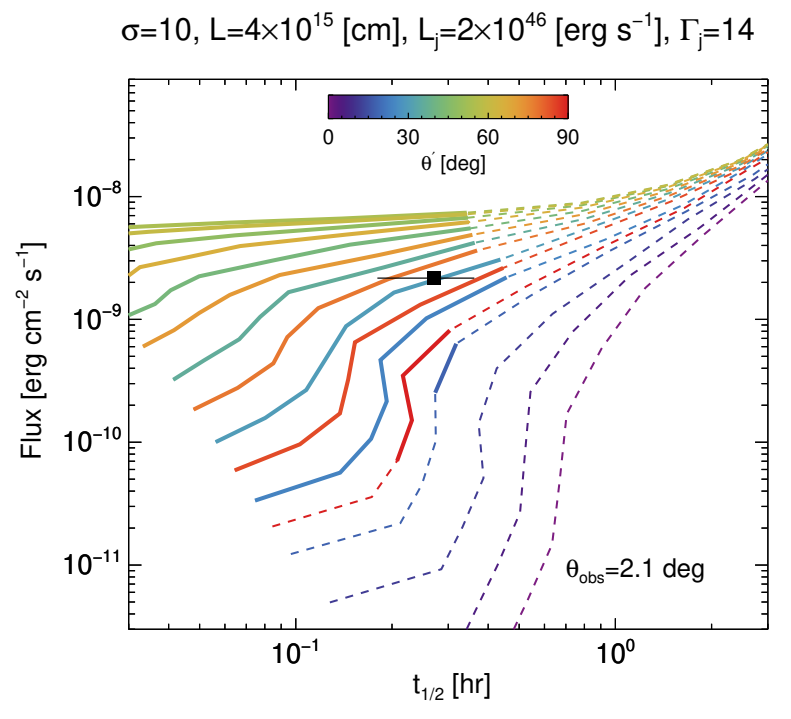

Figure 8. Bolometric peak flux of flares produced by plasmoids of different sizes as a function of the respective fluxdoubling timescale for $\sigma=10$. Colored curves correspond to different orientations (see inset color bar) of the reconnecting layer with respect to the jet axis (for an illustration, see Fig. 3 in Christie et al. 2019). Solid thick lines indicate that the plasmoid Doppler factor $\gtrsim 35$, thus satisfying the $\gamma \gamma$ opacity constraint (see Section 6.1). The peak bolometric flux and flux-doubling time of the fast flare observed in April 152013 is also shown (black symbol). A choice of larger $L^{\prime}$ would shift the curves horizontally to longer timescales, while an increase of $\Gamma_{j}$ would shift the curves diagonally towards the upper left corner of the plot.

Lorentz factor, $\beta_{j} \sim 1, L_{\mathrm{j}}$ is the absolute power of a two-sided jet, $\varpi / L^{\prime}=\epsilon_{\text {rec }} / \Gamma_{\mathrm{j}} \theta_{\mathrm{j}}$ is the cross-sectional radius of the jet, $\epsilon_{\text {rec }} \simeq 0.15$ is the reconnection rate, and $f_{\text {rec }} \simeq 0.5$ is the fraction of dissipated magnetic energy transferred to relativistic pairs (e.g., Sironi et al. 2015). The flux-doubling timescale can be estimated as:

$$
t_{1 / 2} \approx \frac{1}{c \beta_{\mathrm{g}}} \int_{w_{1 / 2}^{\prime}}^{w_{\mathrm{f}}^{\prime}} \frac{\mathrm{d} \tilde{w}}{\delta_{\mathrm{p}}(\tilde{w})} .
$$

where $\delta_{\mathrm{p}}$ is the plasmoid's Doppler factor (see Eq. 8 in PGS16), which evolves during the plasmoid lifetime as this accelerates in the layer. Here, $w_{1 / 2}^{\prime}$ is the size of the plasmoid at the moment the flare luminosity reaches half of its peak value. For details about the derivation of Eqs. 11-12 and assumptions therein, we refer the reader to PGS16. The free parameters of the model are: the plasma magnetization $\sigma$, the orientation of the layer with respect to the jet axis $\theta^{\prime}$, the observer's angle $\theta_{\mathrm{obs}}$, $L^{\prime}, L_{\mathrm{j}}$, and $\Gamma_{\mathrm{j}}$.

We apply the PGS16 model to the fast flaring activity observed in Mrk 421. We use as an illustrative exam- ple the results for 2013 April 15, where fast flares with similar flux-doubling timescales (Table 3 ) have been detected in all energy bands $(3-7 \mathrm{keV}, 7-30 \mathrm{keV}, 30-80$ $\mathrm{keV}, 0.2-0.4 \mathrm{TeV}, 0.4-0.8 \mathrm{TeV},>0.8 \mathrm{TeV})$. Using the peak flux of the fast flare in each energy band, we estimate the peak bolometric luminosity of the fast flare. Given the similar rise timescales in all bands, we use their mean value as the observed $t_{1 / 2}$ of the fast flare (see black symbol in Fig. 8).

We consider a case with plasma magnetization $\sigma=10$. Besides the microphysical parameters, which are benchmarked with PIC simulations of reconnection (see Table 1 in PGS16), we adopt the following values for the free parameters of the model: $L^{\prime}=4 \times 10^{15} \mathrm{~cm}, L_{\mathrm{j}}=$ $2 \times 10^{46} \mathrm{erg} \mathrm{s}^{-1}, \Gamma_{\mathrm{j}}=14$, and $\theta_{\text {obs }}=\left(2 \Gamma_{j}\right)^{-1} \simeq 2.1^{\mathrm{o}}$. Using Eqs. 11 and 12 we can then estimate the peak flux and flux-doubling timescale of flares produced by plasmoids with different sizes, as shown in Fig. 8. We note that the curves showing the different orientations of the reconnecting layer do not depend monotonically on $\theta^{\prime}$ due to the kinematics of plasmoids in the jet (for details, see PGS16). For the adopted parameter values, we find that a larger range of $\theta^{\prime}$ values results in fluxes and flux-doubling timescales that are compatible with the observed values. A choice of larger $L^{\prime}$ would just shift the curves horizontally to longer timescales, while an increase of $\Gamma_{j}$ would shift the curves diagonally towards the upper left corner of the plot (for scalings, see equations (33)-(37) in PSG16). A higher $\sigma$ value would have a similar effect as that of a higher $\Gamma_{\mathrm{j}}$, as it would result in stronger relativistic motions of the plasmoids in the layer. The predicted peak flux depends strongly on the angle at shorter variability timescales, whereas it is almost independent of the orientation of the layer at longer timescales. This merely reflects the fact that flares with longer timescales are produced by the largest plasmoids in the layer that move with non-relativistic speeds in the jet frame. For these plasmoids, the beaming of the radiation is basically determined by $\theta_{\text {obs }}$ and $\Gamma_{j}$. On the contrary, flares with short durations are produced by plasmoids that move relativistically in the jet frame and whose Doppler factor $\delta_{p}$ depends sensitively on both angles $\theta^{\prime}$ and $\theta_{\text {obs }}$.

Flux-flux correlations - Here we examine the flux-flux correlations predicted by the model for the fast and slow components of a plasmoid-powered light curve. To do so, we adopt the results of Christie et al. (2019) and, in particular, the light curves computed for a "vanilla" 


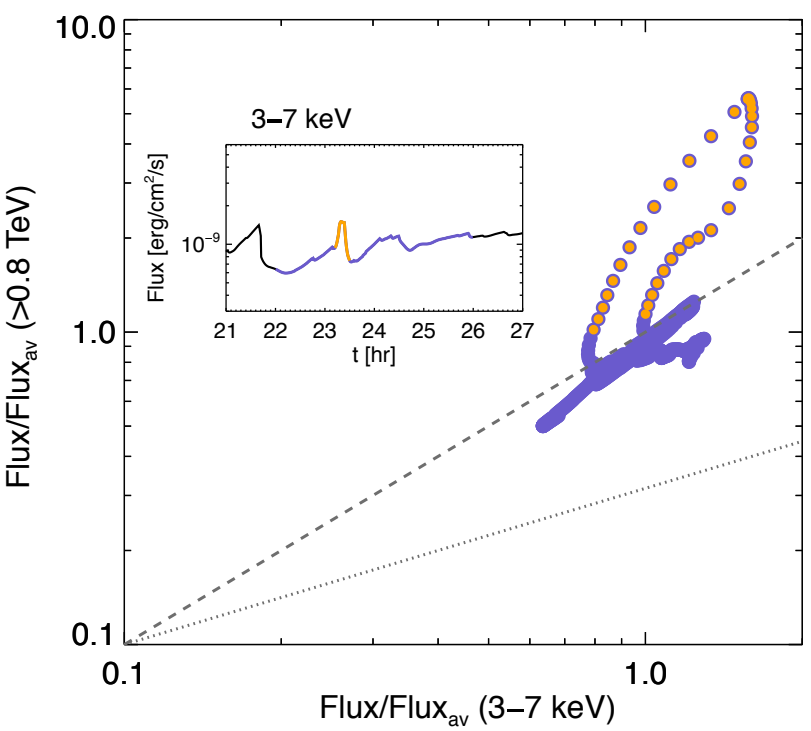

Figure 9. VHE flux ( $>0.8 \mathrm{TeV}$ ) versus X-ray flux (3-7 $\mathrm{keV}$ ) of a plasmoid-powered light curve, computed for a "vanilla" model of a BL Lac source (see model BL10 in Christie et al. 2019). The fluxes are extracted from a 4-hr time window of the total light curve (see purple line in the inset plot) and are normalized to their time-averaged values. The loop-like structure in the flux-flux plot is produced during a fast flare of duration $\sim 0.3 \mathrm{hr}$ (see orange points). Lines with slopes 1 (dashed) and 0.5 (dotted) are overplotted to guide the eye.

model $^{9}$ of a BL Lac-like source for $\sigma=10$ (BL10) with $\theta^{\prime}=30^{\circ}$ and $\theta_{\text {obs }}=0^{\circ}$. The values for the angles correspond to the vanilla $\mathrm{BL}$ Lac model of Christie et al. (2019). A choice of $2.1^{\circ}$ instead of $0^{\circ}$ would have no effect on the conclusions. We focus on a four hour-long segment of the total light curve that displays a fast flare (with duration $\sim 0.3 \mathrm{hr}$ ) emerging on top of a less variable component (see inset plot in Fig. 9). The fluxes computed in the $3-7 \mathrm{keV}$ and $>0.8 \mathrm{TeV}$ energy bands during the selected time window are displayed in the main panel of Fig. 9 (blue points). The fluxes computed during the fast flare are highlighted for clarity (oranged colored points). Although our vanilla model cannot explain the details of the observed correlations shown in Fig. 7, the predicted flux-flux correlations bear some similarities with the observed ones: a tight correlation with slope close to 1 is produced by the slow component of the light curve, whereas the fast flare leads to a looser correlation with a steeper slope.

We note that the temporal resolution of our model light curve is about $30 \mathrm{~s}$. The loop-like structure pro-

9 Performing detailed radiative transfer calculations, as in Christie et al. (2019), for parameters similar to those in Fig. 8 lies beyond the scope of this paper. duced by the fast component would likely be missed in real data due to the coarser sampling. For example, even with the 15-min sampling used in this work, the loop would consist of just two data points. Our results are also in agreement with those shown in Fig. 7, where the loop is not evident in the real data, but becomes visible only when the fitted fluxes of the slow and fast components are plotted.

Caveats - We next discuss some caveats of the plasmoid-dominated reconnection model. The fast component of the optical light curve is predicted to be wider than the one seen in X-rays and VHE gamma rays, due to the longer cooling timescales of electrons radiating in the optical band. Nevertheless, some degree of correlation between the optical and other high-energy bands is expected in this model. Unfortunately, this cannot be tested with our current dataset because we do not have a proper optical coverage around the peak times of the fast (sub-hour) flares in X-rays and VHE gamma rays (see Fig. 1 and Fig. 4, 14, 15, 16). On longer (multihour) timescales, the observed optical flux appears to roughly follow the temporal trends in X-rays and VHE gamma rays for some days (e.g., April 13 and April 17), while it appears to be anti-correlated for some other days (e.g., April 11, April 12, April 15, and April 18). Such variability patterns cannot be explained by the vanilla model we described above. If magnetic reconnection can be triggered in different jet locations, then it is possible to have the formation of multiple layers in the jet with different properties (e.g., sizes, magnetizations, and orientations; Giannios et al. 2009; Giannios \& Uzdensky 2019). It is then possible that the observed optical emission is dominated by a different layer (e.g., larger and with different orientation) than the one producing the high-energy emissions (e.g., smaller and more aligned to the observer).

To fully account for all the observed properties of the flares (i.e., absolute flux and spectra), one would have to adjust some of the parameters entering the vanilla model, such as the properties of the injected particle distribution within each plasmoid (e.g., slope, minimum and maximum Lorentz factor). The vanilla model described above could be improved through the inclusion of continuous slow acceleration of particles within plasmoids due to plasmoid compression (Petropoulou \& Sironi 2018) and/or impulsive particle injection with a harder energy spectrum during plasmoid mergers (Christie et al. 2019).

PGS16 and Christie et al. (2019) developed a radiative model based on the PIC simulations of Sironi et al. (2016). Although the radiative model takes into account the plasmoid kinematics and dynamics, it treats each 
plasmoid as a homogeneous source. Thus, this model cannot be used to predict the polarization signatures of a reconnection event. This issue has been recently tackled in Zhang et al. (2018), where the polarized emission was computed directly from 2D PIC simulations of reconnection, demonstrating that the latter can produce variable optical polarization. Due to the different simulation setups used in Sironi et al. (2016) and Zhang et al. (2018), it is not clear how the polarization results found in the latter study can be applied to the plasmoiddominated model discussed here.

\section{SUMMARY AND CONCLUSIONS}

This manuscript presents results from an extensive multiwavelength campaign in 2013 on the blazar Mrk 421, which included, for the first time, highly sensitive hard X-ray observations with $N u S T A R$. The article focuses on the exceptional flaring activity observed during nine consecutive days, from April 11 to April 19, and complements the results obtained with the low VHE gamma-ray activity from January to March 2013, which was reported in Baloković et al. (2016). The 2013-April flaring activity is the brightest detected with MAGIC to date, and the second brightest observed in Mrk 421 to date, after the 2010-February flaring activity reported in VERITAS-MAGIC-2019 (ApJ publication in press). More important than the bright blazar activity, is the comprehensive multi-instrument dataset collected during these nine days, which includes 42 hours with MAGIC, 27 hours with VERITAS, 18 hours with Swift, and 71 hours with $N u S T A R$, out of which 43 hours were taken simultaneously to the VHE observations from MAGIC and VERITAS.

Using simultaneous MAGIC-VERITAS observations of Mrk 421 during this high activity, we noticed that the VHE gamma-ray flux values from VERITAS are systematically lower than those from MAGIC throughout the flaring activity. The flux difference is energy dependent, being about $10 \%$ in the $0.2-0.4 \mathrm{TeV}$ band, and about $30 \%$ in the $>0.8 \mathrm{TeV}$ band. These differences are within the quoted systematic uncertainties from both instruments, and probably related to the absolute energy reconstruction. In order to perform variability and correlation studies during single days, the VERITAS flux values were scaled up to match those from MAGIC (see Appendix A). The physics results reported in this manuscript relate to the variations in the VHE gammaray flux and its correlation to the X-ray flux, and hence do not depend on the absolute value of the VHE gammaray flux. The results reported here would essentially be the same if we had scaled down the MAGIC fluxes to match the VERITAS fluxes.
Owing to the large fluxes and the unprecedented coverage provided by the simultaneous NuSTAR and MAGIC/VERITAS observations during these nine consecutive days, this dataset allowed us to evaluate the variability and correlations over three X-ray bands (3-7 keV, 7-30 keV and 30-80 keV) and three VHE gamma-ray bands $(0.2-0.4 \mathrm{TeV}, 0.4-0.8 \mathrm{TeV}$ and $>0.8 \mathrm{TeV}$ ) on timescales of 15 minutes, producing the most detailed X-ray/VHE variability and correlation study of Mrk 421 to date. This study yielded a number of results, which we summarise below:

- The fractional variability $F_{v a r}$ vs. energy for the 9-day flaring activity shows a similar pattern to that during the low X-ray and VHE gamma-ray activity from Baloković et al. (2016). The $F_{v a r}$ vs energy shows a double-bump structure with the highest variability occurring in the X-ray and VHE gamma-ray bands, while the variability in radio and optical bands is very low. Additionally, we find that $a$ ) the $F_{\text {var }}$ values for the highest $\mathrm{X}$-ray and VHE energies during this 9-day flaring activity are much higher than those from the low activity reported in Baloković et al. (2016); b) the $F_{\text {var }}$ values obtained with data from the single nights are much smaller than those obtained with the 9-day flaring activity, indicating that the processes with timescales larger than half day dominate the variability at X-ray and VHE over the processes that have timescales of hours; and c) $F_{\text {var }}$ typically increases with energy for single nights, in the same way as observed for multi-day or multi-month timescales; but we also find nights where $F_{\text {var }}$ does not increase (or even decreases) with energy, hence suggesting the existence of distinct mechanisms (or distinct particle populations and/or regions) responsible for the variability of Mrk 421.

- There is significant variability in the optical emission, as well as in the polarization degree and EVPA (see Fig. 1), but they are not correlated to the X-ray and VHE gamma-ray emission. The optical polarization variations, while statistically significant, relate to very low polarization degree (typically less than 5\%) and appear to be random and without any obvious coherent structure. This is consistent with a multi-zone scenario such as the one proposed in Marscher (2014), where the sum of polarization vectors from many zones would result in a low level of polarization with random fluctuations in both the polarization degree and the EVPA. At $86 \mathrm{GHz}$, the polarization degree is also 
low (about 3\%), as it happens with the optical data, but the polarization angle differs by about $70^{\circ}$, which indicates that the radio and optical emissions are produced, or at least dominated, by different regions along the jet of Mrk 421.

- The flux variability measured at $\mathrm{GeV}$ is dominated by the large flux variation around April 15, which is the day with the highest X-ray activity. Despite the lower sensitivity of Fermi-LAT, in comparison to that of the X-ray and VHE gamma-ray instruments, the GeV flux of Mrk 421 can be measured significantly on 12-hour timescales, and it does not show the large flux variations (by factors of a few) that are reported for the X-ray and VHE gammaray energies. Therefore, this observation indicates that, when considering timescales of days, there is no correlation between the $\mathrm{GeV}$ band and the $\mathrm{keV}$ and $\mathrm{TeV}$ bands.

- Differently to the January-to-March 2013 results reported in Baloković et al. (2016), the light curves from the April 2013 flaring activity show substantial intra-day variability in both the X-ray and VHE gamma-ray bands. Moreover, one can clearly distinguish variability on sub-hour timescales on the top of flux variations occurring on multi-hour timescales. The intra-night flux variations were quantified with a function consisting of an exponential increase and decay (fast component) on the top of a monotonically increasing or decreasing flux (slow component). We found out that, within the X-ray and VHE gamma-ray bands, the parameters describing the fast component do not depend on energy, while the ones describing the slow component can depend strongly on the energy. We also found out that the fast component is symmetric (rise time $\sim$ decay time). This suggests that the mechanisms that dominate the production of the sub-hour flux variability appear to be "achromatic", while those responsible for the production of the multi-hour flux variability can be "chromatic", at least in some cases.

- A lower limit to Doppler factor of 35 was derived by requiring that the emitting region producing the fastest VHE variability is optically thin to $\gamma \gamma$ pair production (see Section 6.1). This limit implicitly assumes that the low-energy (optical/IR) and VHE gamma-ray photons are produced in the same region. However, the multi-band variability and correlations derived with the 9-day April 2013 flaring activity indicates that these two emissions are likely originated in different regions, which would relax the derived lower limit.

- Using the parameters from the fast component, we find that there are no delays between the X-ray and VHE gamma-ray emission down to the resolution of our measurement (3 minutes during April 15th). This is the strongest constraint on the correlated behavior between X-ray and gamma rays in Mrk 421, and among the strongest constraints derived with $\mathrm{TeV}$ blazar data until now.

- The correlation between VHE gamma rays and $\mathrm{X}$-rays is positive (i.e., the VHE gamma-ray emission increases when the X-ray emission increases); but there are multiple flavors in the strength and characteristics of this correlated behavior that change both across energy (even for nearby energy bands) and over time (on day timescales). The strongest correlation occurs between the lowest X-ray band (3-7 keV) and the highest VHE gamma-ray band $(>0.8 \mathrm{TeV})$, where one finds an approximately linear change in the VHE flux with the X-ray flux. On the other hand, the weakest correlation occurs between the highest $\mathrm{X}$-ray band $(30-80 \mathrm{keV})$ and the lowest VHE gamma-ray band $(0.2-0.4 \mathrm{TeV})$, where the VHE flux changes with the X-ray flux with a slope of $\sim 0.3$ in log-log scale. This indicates that the particle population dominating the emission in the $3-7 \mathrm{keV}$ and $>0.8 \mathrm{TeV}$ bands are closely related, while this does not occur for the bands $30-80 \mathrm{keV}$ and $0.2-0.4 \mathrm{TeV}$. The decrease in the magnitude and significance of the correlation (with respect to the maximum) when increasing the X-ray energy and decreasing the VHE gamma-ray energy is expected if the variability is dominated by the highest energy electrons; but the rapid change in the correlation pattern with energy is remarkable, and it has never been observed to date neither for Mrk 421 nor for any other $\mathrm{TeV}$ blazar.

- Within the context of a synchrotron/SSC model, the approximately linear correlation between VHE and X-ray could be an indication of an expanding blob with a decreasing magnetic field (Katarzyński et al. 2005). It could also be an indication of more than one emitting region (Fossati et al. 2008).

- The temporal and spectral properties of the multiband flares disfavor a single-zone interpretation of the results (see Section 6). A scenario with multiple zones (and possibly with narrow electron energy distributions) is likely needed to explain 
the achromatic (chromatic) variability of the fast (slow) component of the light curves, as well as the changes of the flux-flux correlation within daylong timescales. We showed that plasmoids forming in the jet of Mrk 421 due to magnetic reconnection might explain some of the main observational results of this campaign. In this scenario, the multi-hour flux variations, which are found to be chromatic for some nights, would be dominated by the combined emission from various plasmoids of different sizes and velocities, while the sub-hour flux variations, which appear to be achromatic, would be dominated by the emission from a single small plasmoid moving with relativistic speeds along the magnetic reconnection layer. Due to the different origin of the fast and slow components of plasmoid-powered light curves, the flux correlations between different energy bands are also expected to differ. In particular, the reconnection model predicts tight correlations (with linear or sub-linear relation between energy bands) for the slow component of the light curve, and weaker correlations (with steeper than linear relation between fluxes) during fast flares. However, the characteristic loop-like structure in the VHE flux vs. $\mathrm{X}$-ray flux plot of predicted for fast flares is likely to be missed with the realistic temporal binning.

The accuracy and the level of detail of this study, which is unmatched among all VHE gamma-ray emitting blazars (including past observations of Mrk421), shows a large degree of complexity in the variability and correlation patterns. This complexity may be present in other blazars, but it may be difficult to observe owing to insufficient temporal and energy coverage in the observations, especially with the instruments that can resolve the VHE gamma-ray fluxes with high precision. The study presented here on Mrk 421 sheds some light into this complex behavior, and represents a pathfinder to the studies that may be done with the next generation of ground-based VHE gamma-ray instruments like the Cherenkov Telescope Array (CTA), which is expected to resolve many VHE blazars with a level of accuracy comparable to that of MAGIC and VERITAS to resolve Mrk 421 during this period of outstanding activity.

The MAGIC collaboration would like to thank the Instituto de Astrofísica de Canarias for the excellent working conditions at the Observatorio del Roque de los Muchachos in La Palma. The financial support of the German BMBF and MPG, the Italian INFN and INAF, the Swiss National Fund SNF, the ERDF under the
Spanish MINECO (FPA2017-87859-P, FPA2017-85668P, FPA2017-82729-C6-2-R, FPA2017-82729-C6-6-R, FPA2017-82729-C6-5-R, AYA2015-71042-P, AYA201676012-C3-1-P, ESP2017-87055-C2-2-P, FPA201790566REDC), the Indian Department of Atomic Energy, the Japanese JSPS and MEXT, the Bulgarian Ministry of Education and Science, National RI Roadmap Project DO1153/28.08.2018 and the Academy of Finland grant nr. 320045 is gratefully acknowledged. This work was also supported by the Spanish Centro de Excelencia "Severo Ochoa" SEV-2016-0588 and SEV-20150548, and Unidad de Excelencia "María de Maeztu" MDM-2014-0369, by the Croatian Science Foundation (HrZZ) Project IP-2016-06-9782 and the University of Rijeka Project 13.12.1.3.02, by the DFG Collaborative Research Centers SFB823/C4 and SFB876/C3, the Polish National Research Centre grant UMO2016/22/M/ST9/00382 and by the Brazilian MCTIC, CNPq and FAPERJ.

The Fermi-LAT Collaboration acknowledges generous ongoing support from a number of agencies and institutes that have supported both the development and the operation of the LAT as well as scientific data analysis. These include the National Aeronautics and Space Administration and the Department of Energy in the United States, the Commissariat à l'Energie Atomique and the Centre National de la Recherche Scientifique / Institut National de Physique Nucléaire et de Physique des Particules in France, the Agenzia Spaziale Italiana and the Istituto Nazionale di Fisica Nucleare in Italy, the Ministry of Education, Culture, Sports, Science and Technology (MEXT), High Energy Accelerator Research Organization (KEK) and Japan Aerospace Exploration Agency (JAXA) in Japan, and the K. A. Wallenberg Foundation, the Swedish Research Council and the Swedish National Space Board in Sweden. Additional support for science analysis during the operations phase is gratefully acknowledged from the Istituto Nazionale di Astrofisica in Italy and the Centre National d'Études Spatiales in France.

This work made use of data from the NUSTAR mission, a project led by the California Institute of Technology, managed by the Jet Propulsion Laboratory, and funded by the National Aeronautics and Space Administration. We thank the NuSTAR Operations, Software, and Calibration teams for support with the execution and analysis of these observations. This research has made use of the NuSTAR Data Analysis Software (NuSTARDAS) jointly developed by the ASI Science Data Center (ASDC, Italy) and the California Institute of Technology (USA). 
This research has made use of the XRT Data Analysis Software (XRTDAS) developed under the responsibility of the ASI Science Data Center (ASDC), Italy.

D.P. and A.B. are grateful to Amy Furniss and Wystan Benbow for providing the VERITAS VHE gammaray fluxes and for useful discussions about them. M.P. acknowledges support from the Lyman Jr. Spitzer Postdoctoral Fellowship and NASA Fermi grant No. 80NSSC18K1745., and J.F. is partially supported by NASA under contract S-15633Y. M.B. acknowledges support from NASA Headquarters under the NASA Earth and Space Science Fellowship Program (grant NNX14AQ07H) and the Black Hole Initiative at Harvard University, which is funded in part by the Gordon and Betty Moore Foundation (grant GBMF8273) and in part by the John Templeton Foundation.

This research was partially supported by the Bulgarian National Science Fund of the Ministry of Education and Science under grants DN 18-13/2017, DN 18-10/2017, KP-06-H28/3 (2018) and KP-06-PN38/1 (2019), as well as for the Spanish MIMECO (AYA201680889-P, RYC-2013-14511) and the IAA-CSIC "Severo
Ochoa" program SEV-2017-0709. The St. Petersburg University team acknowledges support from Russian Science Foundation grant 17-12-01029. The Abastumani team acknowledges financial support of the project FR/638/6-320/12 by the Shota Rustaveli National Science Foundation under contract 31/77. T.G. acknowledges support from Istanbul University (Project numbers 49429 and 48285), Bilim Akademisi (BAGEP program), and TUBITAK (project numbers 13AT100431, 13AT100-466, and 13AT60-430). The Boston University effort was supported in part by NASA grants NNX12AO90G and NNX14AQ58G. Data from the Steward Observatory spectropolarimetric monitoring project were used in this paper. This program is supported by Fermi Guest Investigator grants NNX08AW56G, NNX09AU10G, NNX12AO93G, and NNX15AU81G. The OVRO $40 \mathrm{~m}$ monitoring program is supported in part by NASA grants NNX08AW31G and NNX11A043G and NSF grants AST-0808050 and AST-1109911. The Metshovi team acknowledges the support from the Academy of Finland to our observing projects (numbers 212656, 210338, 121148, and others).

\section{APPENDIX}

\section{A. QUANTIFICATION AND CORRECTION OF THE OFFSET BETWEEN MAGIC AND VERITAS FLUX MEASUREMENTS}

The high accuracy in the VHE gamma-ray flux measurements presented in this work reveals an offset in the simultaneous MAGIC and VERITAS fluxes. This offset, which is energy-dependent and can reach up to $30 \%$, is within the flux systematic uncertainties reported by each instrument (on the order of 20-25\%). The factor that dominates the systematic uncertainties in an IACT is the uncertainty on the absolute energy scale calibration. The energy reconstruction of VHE gamma-ray showers by IACTs relies heavily on Monte Carlo simulations. The main source of systematic uncertainty on the energy scale is the uncertainty on the "light yield" (i.e. the total light throughput) considered in these simulations, associated for instance to the average transparency of the atmosphere or the light collection efficiency of the telescopes. Each instrument tunes these parameters over long periods of time with stable performance (during years). For this reason, deviations with respect to the considered atmosphere and telescope models are expected for shorter time-scales on the order of $15 \%$ (as discussed in Madhavan \& for the VERITAS Collaboration (2013); Aleksić et al. (2016)).

In order to exploit the excellent coverage of this dataset and perform intra-night VHE gamma-ray flux variability and X-ray/VHE flux correlation studies, these energy-dependent offsets need to be corrected. This mismatch shows up prominently when comparing single-night light curves. As an example, the left panel in Fig. 10 displays the multi-band VHE light curves during the night of April 12th, showing an increasing offset with energy reaching about $30 \%$ for the VHE gamma-ray fluxes with $\mathrm{E}>0.8 \mathrm{TeV}$. We note that an increasing offset with energy is expected if the main source of this systematic difference between the MAGIC and VERITAS fluxes is the energy scale.

The simplest approach to correct for this difference is to scale the lightcurves of one of the instruments by an energy-dependent factor. As we have been using throughout this work three different energy ranges (i.e. $0.2-0.4 \mathrm{TeV}$, $0.4-0.8 \mathrm{TeV}$, and $>0.8 \mathrm{TeV}$ ), three different flux ratios need to be calculated to properly correct for this bias. To calculate these correction factors, two different methods were tested: a) obtain three scaling factors by normalising the MAGIC and VERITAS fluxes in the three energy bands; and b) calculate the energy-scale correction that makes MAGIC and VERITAS spectra compatible, and then obtain the three scaling factors for the three energy bands considered. The first approach is purely agnostic, while the second one assumes that the small mismatch between 

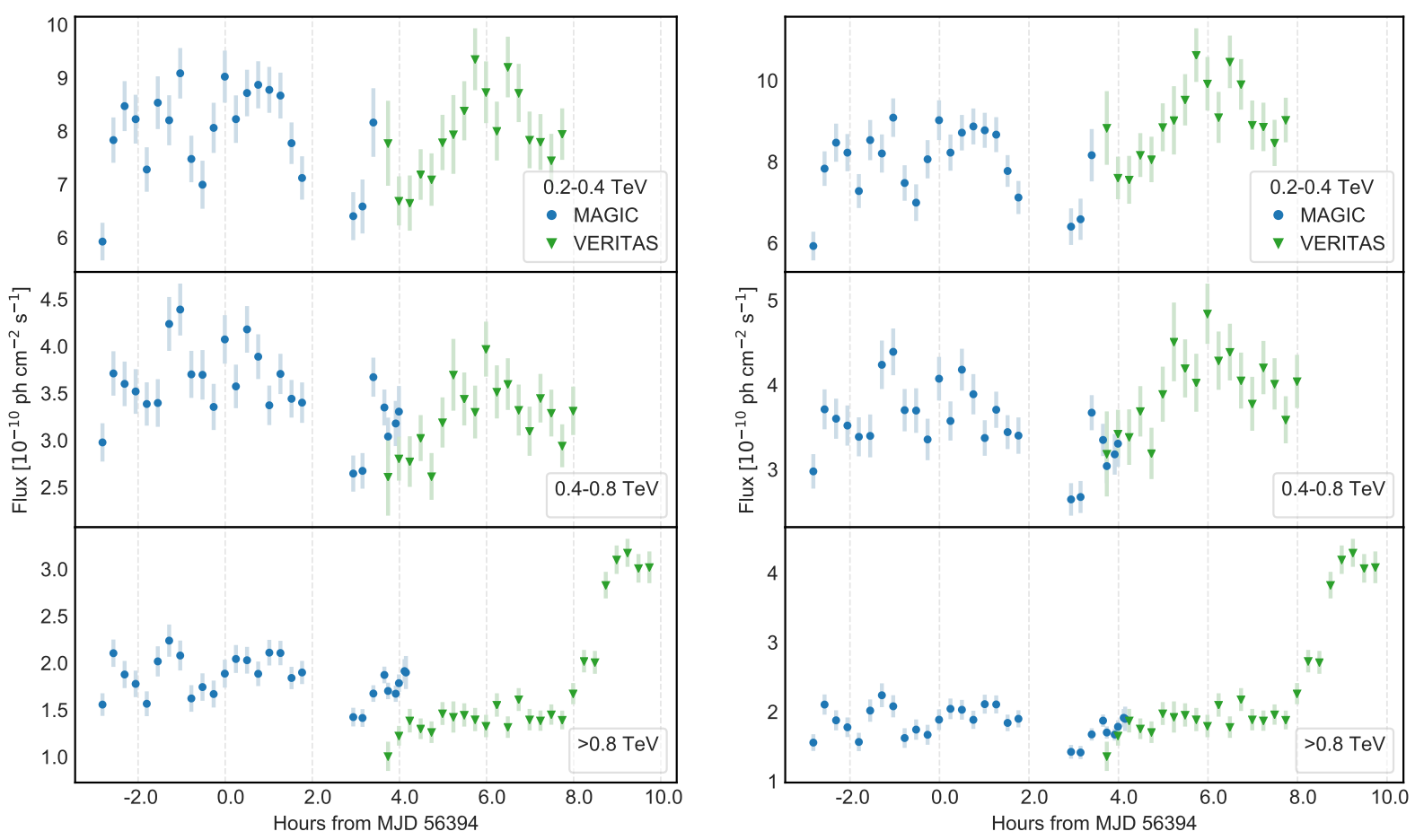

Figure 10. Light curves from April 12th in three VHE gamma-ray energy bands: $0.2-0.4 \mathrm{TeV}, 0.4-0.8 \mathrm{TeV}$ and $>0.8 \mathrm{TeV}$, as measured with MAGIC and VERITAS before (left) and after (right) applying the correction factors from Table 5 . The temporal coverage (i.e. number of data points) varies with the energy band because of the increasing energy threshold with the increasing zenith angle of the observations (see Sect. 2 for further details).

MAGIC and VERITAS VHE gamma-ray measured fluxes is dominated by the systematic uncertainty on the energy scale of each instrument. Both strategies make use of the $2 \mathrm{~h} 45 \mathrm{~min}$ simultaneous MAGIC and VERITAS observations taken during four different nights (see Tab. 1).

Inferring the correction factor from available simultaneous data is the most direct method to calculate these factors; but it cannot be applied to the $0.2-0.4 \mathrm{TeV}$ energy band because the simultaneous MAGIC-VERITAS data relate to MAGIC observations at zenith angle above $55^{\circ}$, which have an analysis energy threshold above $0.3 \mathrm{TeV}$ (see Sect. 2). Fig. 11 shows a comparison of the VHE gamma-ray fluxes (in the two available energy bands) derived with simultaneous 15-min time bins, which span throughout four consecutive nights: from April 12 to April 15.

The figure shows two important characteristics of this offset. First, the ratio at a given energy band is approximately the same for all the simultaneous observations, hence indicating a systematic effect between MAGIC and VERITAS, which is not related to the peculiarity of one single day (e.g. bad weather in one of the two telescope sites). Second, the ratio of MAGIC and VERITAS fluxes deviates from 1 by a larger amount in the highest energy band, hence confirming the energy dependence of the offset. The correction factors and uncertainties inferred with this method are reported in the first column of Table 5 .

As described in Aleksić et al. (2016), MAGIC analysis allows to test the impact of a modified light yield over a given reconstructed spectral energy distribution (SED). By applying relative light yield corrections to the MAGIC data simultaneous with VERITAS, we are able to test which value minimizes the MAGIC-VERITAS SED differences. This search determined that a decrease of $20 \%$ in the MAGIC light makes the simultaneous MAGIC and VERITAS spectra compatible ${ }^{10}$. The flux correction factors for the three energy ranges considered here were then computed through the ratio of the pre- and post-light-yield-corrected MAGIC lightcurves in the three energy bands. In order to reduce the statistical uncertainties, we used the full MAGIC data set from these four nights (April 12-15), instead

${ }^{10}$ A similar effect is expected while increasing the light yield of VERITAS, but these tests were only possible over MAGIC data. 

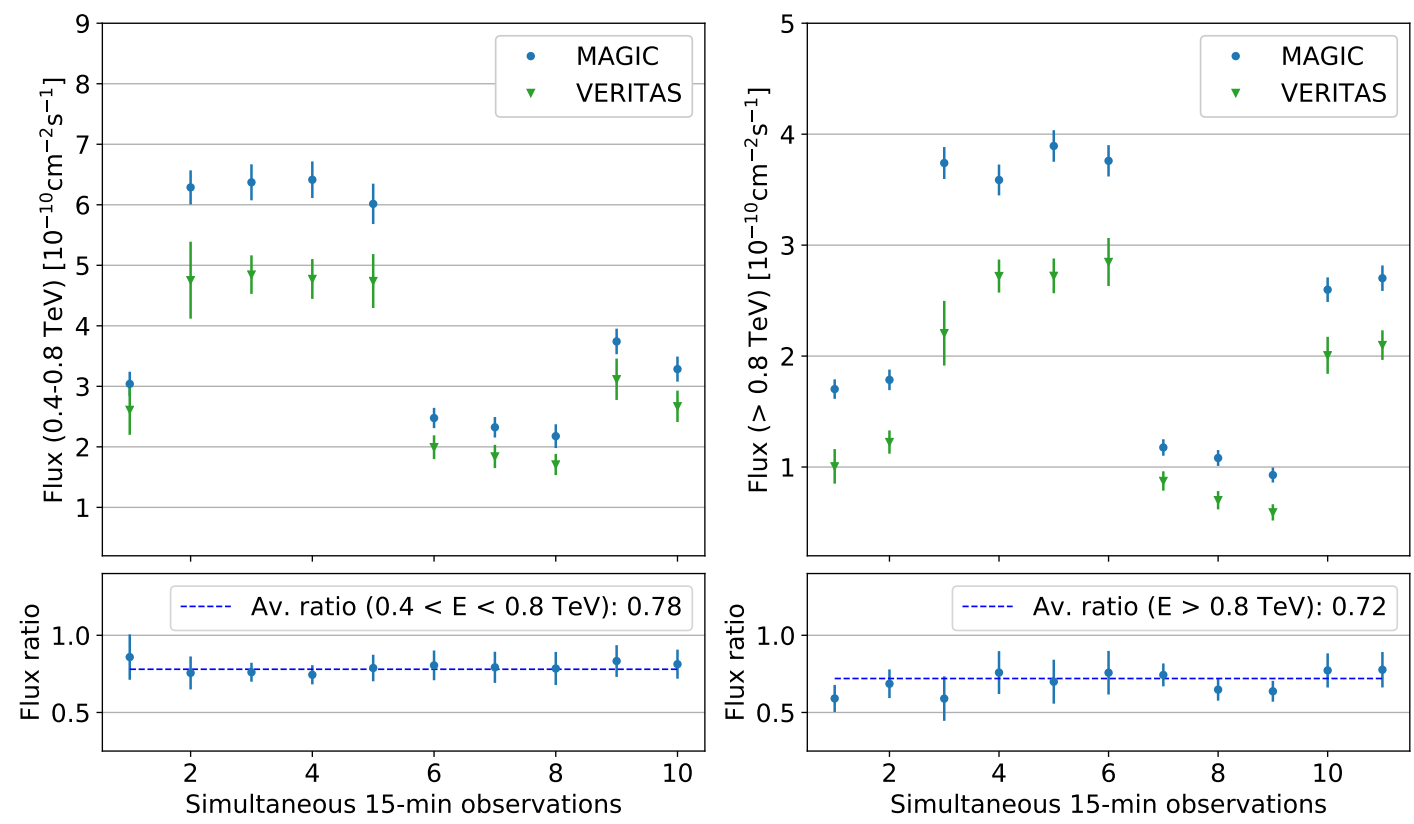

Figure 11. Comparison of the VHE gamma-ray fluxes measured with MAGIC and VERITAS for the 15-min time intervals of simultaneous MAGIC-VERITAS observations.

Table 5. Scaling factors (defined as $F_{\text {VERITAS }} / F_{\text {MAGIC }}$ ) inferred from two different methods (see text for further details) for the three VHE gamma-ray energy bands considered. The values reported correspond to the best fit value and the related statistical uncertainty. Both methods use data from simultaneous MAGIC-VERITAS observations.

\begin{tabular}{c|c|c}
\hline Energy band & Flux ratio & Energy-scale corr. \\
\hline $0.2-0.4 \mathrm{TeV}$ & - & $0.88 \pm 0.03$ \\
$0.4-0.8 \mathrm{TeV}$ & $0.78 \pm 0.03$ & $0.82 \pm 0.06$ \\
$>0.8 \mathrm{TeV}$ & $0.72 \pm 0.02$ & $0.74 \pm 0.05$ \\
\hline
\end{tabular}

of using only the time intervals with simultaneous MAGIC-VERITAS observations. The flux ratios derived with this method are reported in the second column of Table 5.

As shown in Table 5, there is a very good agreement in the flux correction factors calculated with these two different methodologies. From here on, the VERITAS light curves are normalized to those of MAGIC using the method derived with the energy-scale correction test (second column in Table 5). We chose this method (instead of applying the flux ratio method) because this one provides a scaling factor for the lowest energy band, 0.2-0.4 TeV, for which we do not have simultaneous MAGIC-VERITAS data. An example of a light curve after the application of the scaling factors to the VERITAS fluxes is given in the right panel of Fig. 10.

In order to test the impact of the scaling factor uncertainty on the VHE/X-ray correlations reported in Section 5 and Tables 4 and 9, we scaled the VERITAS fluxes by a value drawn from a normal distribution with mean and sigma given by the parameters in Table 5. This was repeated 1000 times, and each time all the correlations were calculated. The standard deviation of the obtained set of correlation parameters was taken as a measure of the systematic uncertainty associated to the scaling of the VERITAS fluxes. For the nine-day correlations in Table 4, the systematic uncertainty was found to be an order of magnitude smaller than the statistical uncertainty. This is expected because the fluxcorrections are of the order of $20 \%$, while the nine-day flaring activity is dominated by flux changes of about one order of magnitude. On the other hand, the impact of this flux scaling is not negligible for quantities derived with the single-night light curves, which are dominated by flux changes of about a factor of 2 . The uncertainties related to the flux-scaling are reported as the second uncertainty in Table 9. Typically, these systematic uncertainties are found to be smaller, or at most comparable, to the statistical uncertainty. 


\section{B. DAILY NORMALIZED LIGHT CURVE SETS}

Using the scaling factors from Appendix A, one can now up-scale all the VERITAS flux measurements and produce single-night light curves with simultaneous VHE and X-ray data spanning about 8-10 hours. The VHE flux measurements can be best compared with the NuSTAR measurements on timescales of 15 minutes, through light curves where the X-ray and VHE gamma-ray fluxes are normalized so that one can compare the relative differences among them.

In order to enable direct (normalized) flux comparisons between the X-ray and VHE bands, the fluxes are normalized by calculating the average flux for each night and each band using only data points that have simultaneous VHE and X-ray observations (filled markers in Fig. 12). The resulting normalization factors are reported in the Table 6. Subsequently, all the light curve fluxes (including the non-simultaneous data marked with open symbols) are divided by this normalization factor. To be able to visually compare the intra-night flux changes, all VHE and X-ray band combinations for each day are shown in Fig. 12. Using the normalization factors from the Table 6, the scaling factors from Table 5, and the normalized light curves flux points from Fig. 12, one can retrieve the primal X-ray and VHE light curves from NuSTAR, MAGIC and VERITAS.

Normalized light curves in Fig. 12 all show that, during these few-days-long activity, the X-ray and VHE emissions of Mrk 421 show quite a number of structures on timescales from multiple hours, and down to timescales smaller than one hour. They also show remarkable correlations in some of the band combinations for selected nights (e.g. 3-7 keV and $>0.8 \mathrm{TeV}$ bands on 15 April, or 3-7 keV and 0.2-0.4 TeV bands on 11 April). Looking at single day band combinations plots, one can also explicitly see different trends for the fast and the slow components (e.g. 15 April slow change in 3-7 keV flux is not as perfectly matched in $0.2-0.4 \mathrm{TeV}$ band as is in the highest VHE band, while the fast component shows comparable agreement). 
15 April 2013

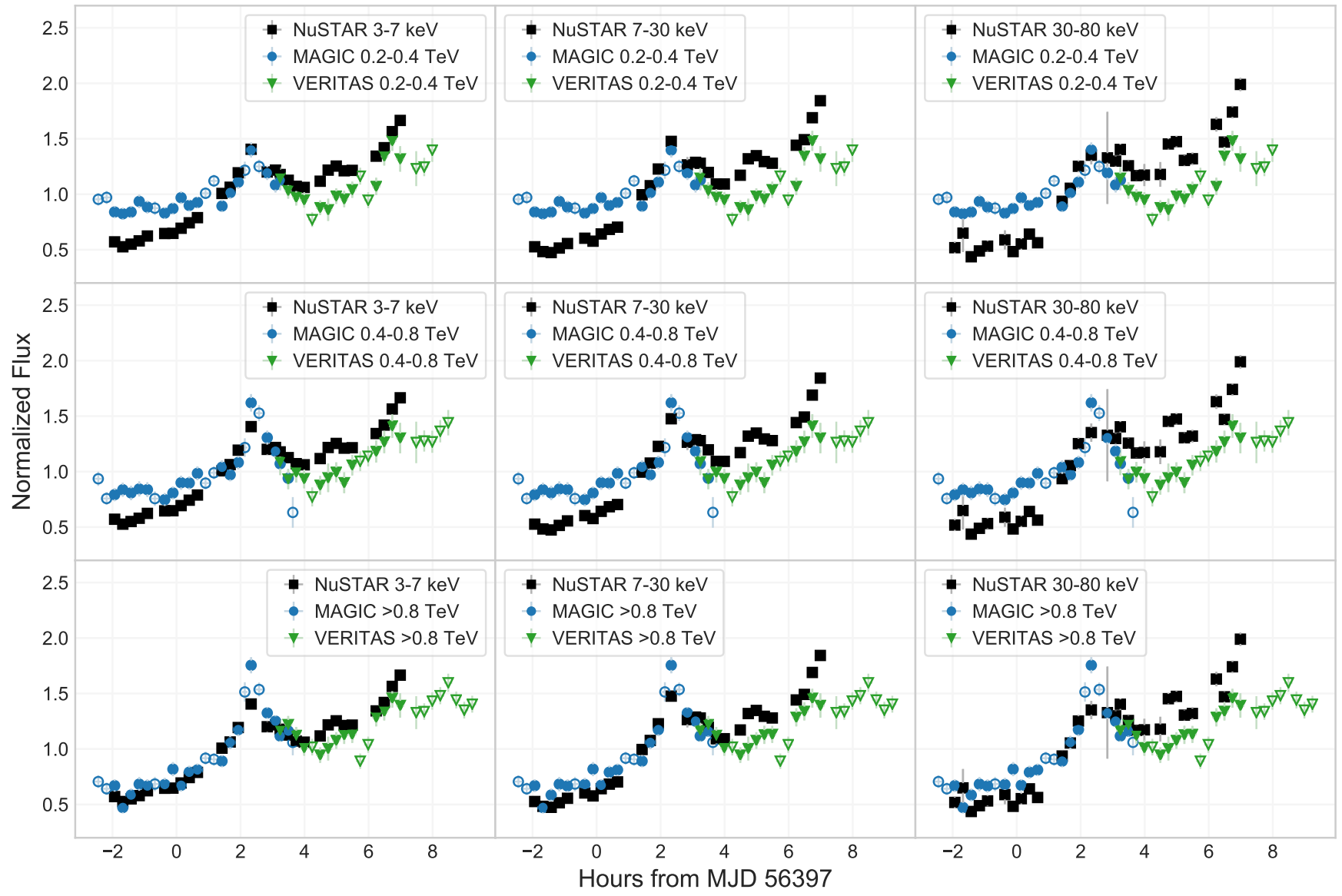

Figure 12. Normalized VHE and X-ray light curves with 15-min time bins for 2013 April 11-19 (continuing on following pages). Successive panels show VHE light curves in three energy bands, namely $E>0.8 \mathrm{TeV}, 0.4-0.8 \mathrm{TeV}$, and $0.2-0.4 \mathrm{TeV}$ energy bands, and three X-ray bands, namely $3-7 \mathrm{keV}, 7-30 \mathrm{keV}$ and $30-80 \mathrm{keV}$. Filled markers are used for those 15-min temporal bins with simultaneous X-ray and VHE data. The fluxes are normalized with the average flux for the night, where the average flux is computed excluding the non-simultaneous X-ray and VHE observation. 

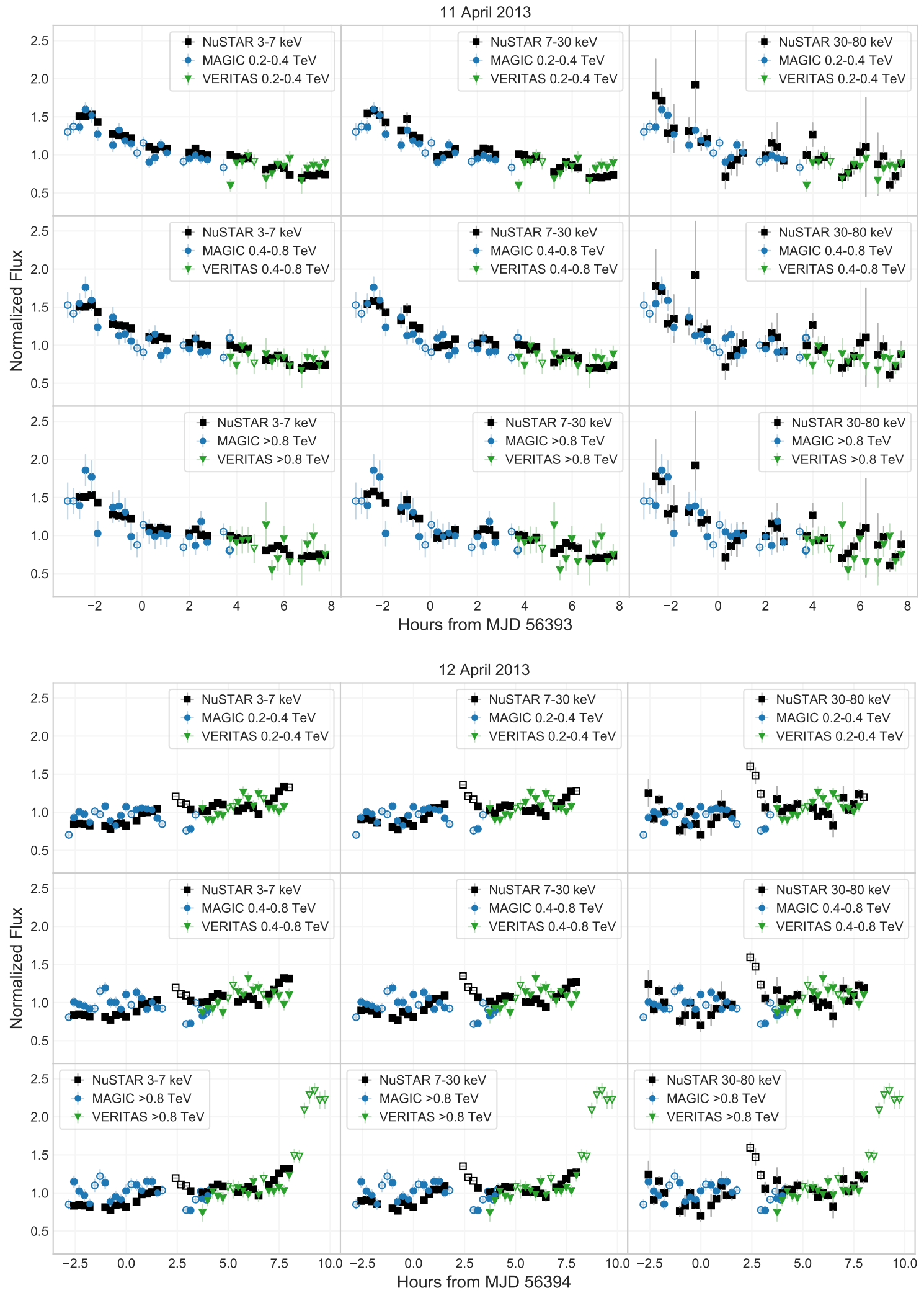

Figure 12 continued 

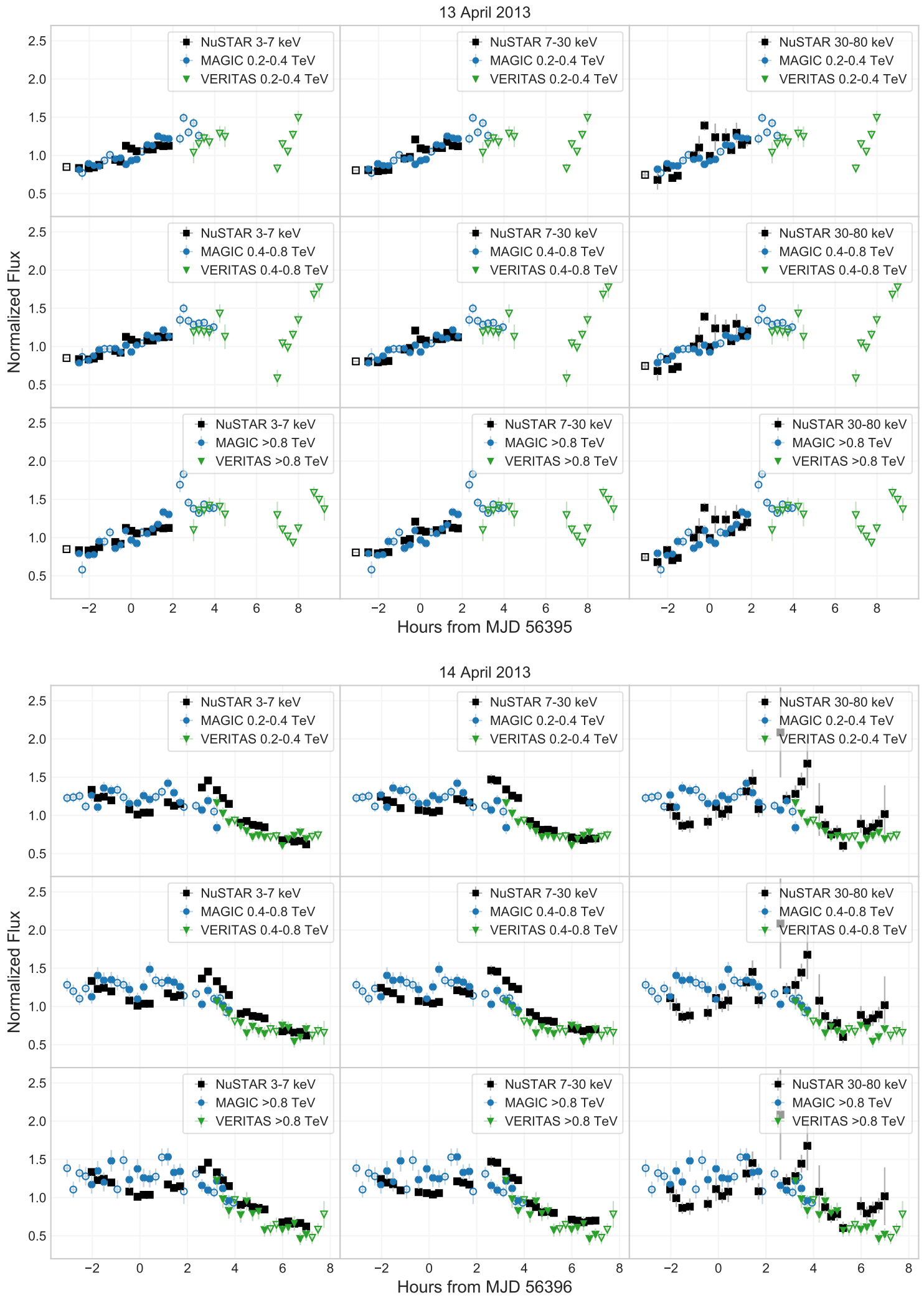

Figure 12 continued 

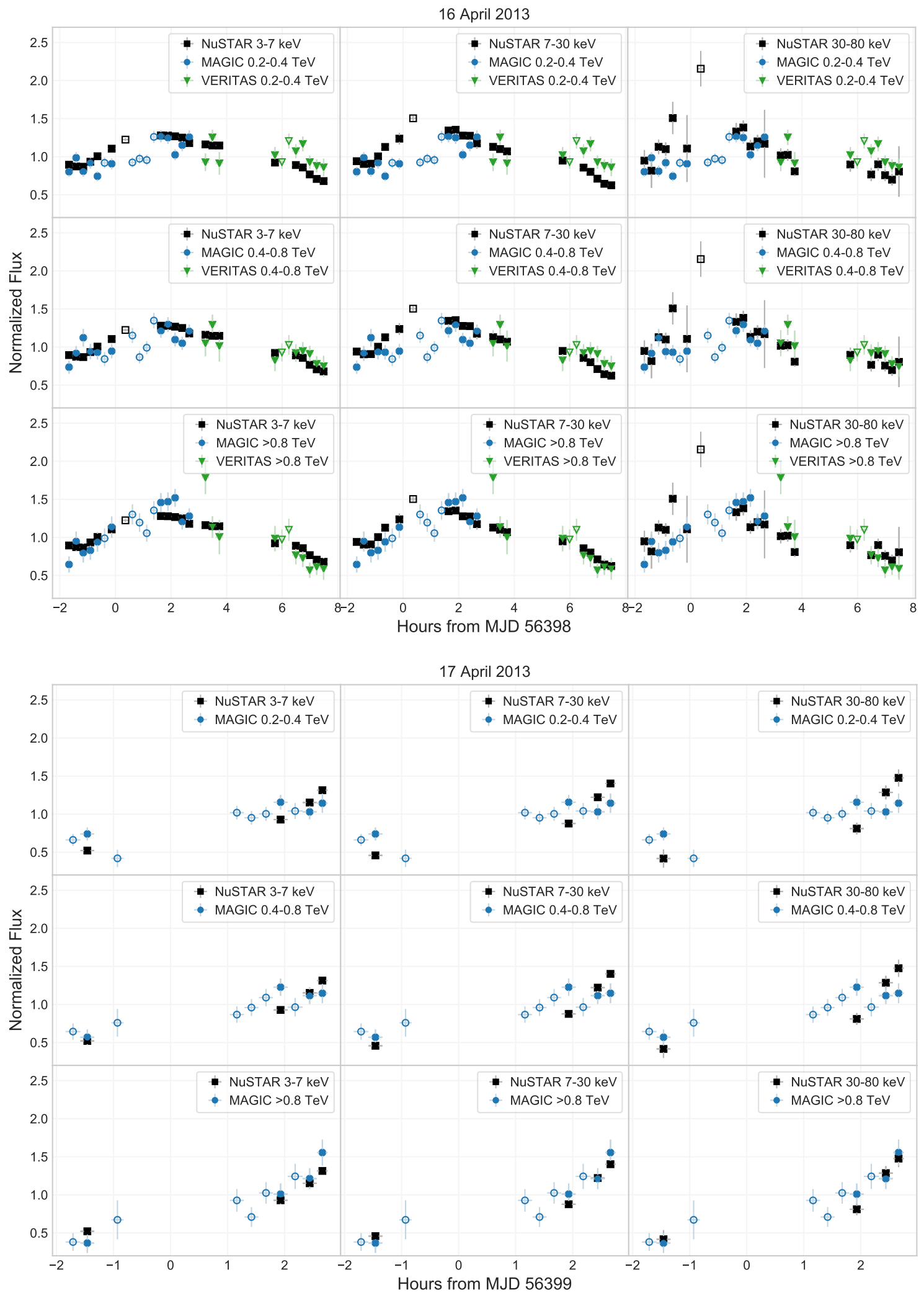

Figure 12 continued 

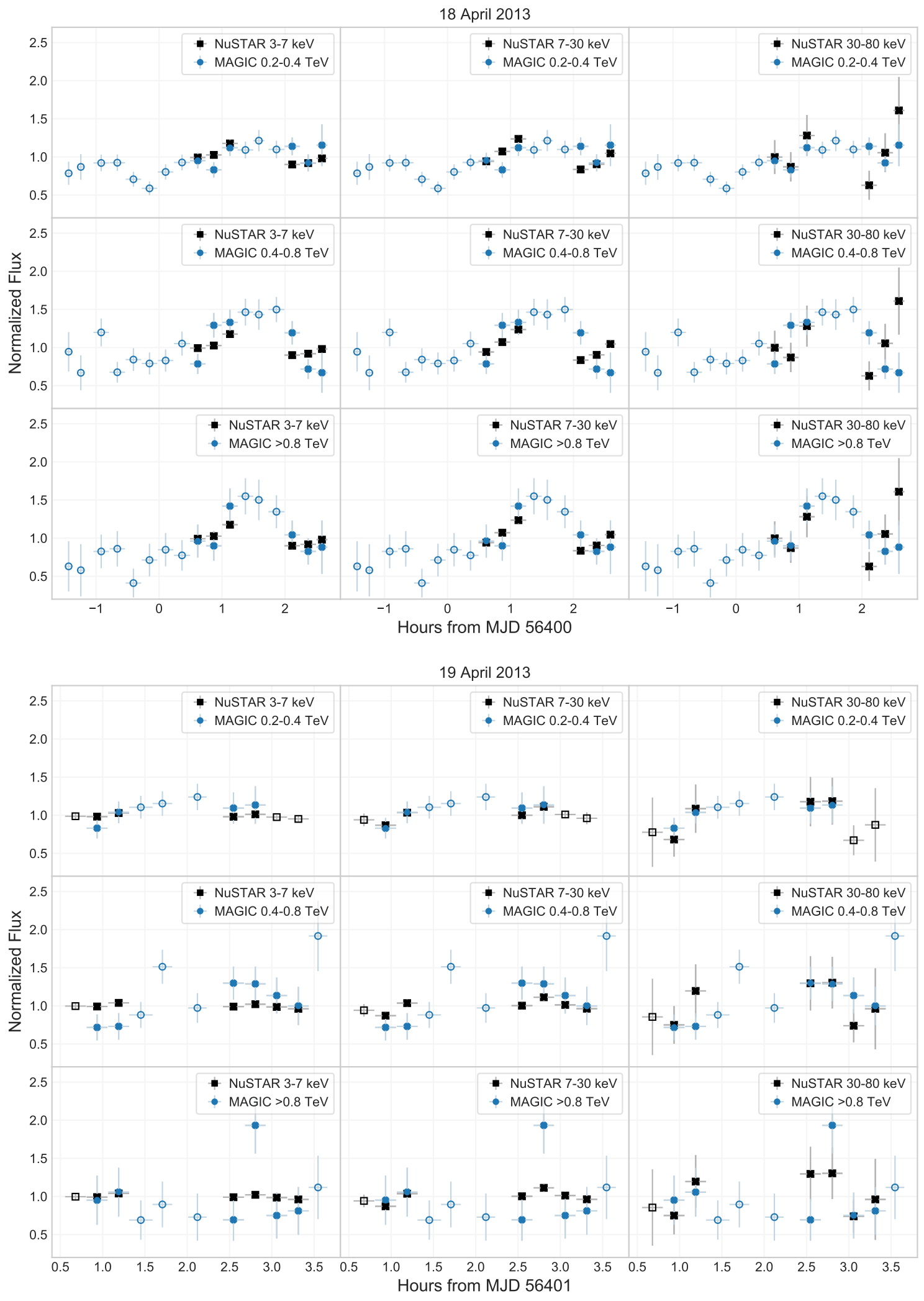

Figure 12 continued 
Table 6. Normalization factors ${ }^{\mathrm{a}}$ used in the daily light curves reported in Fig. 12.

\begin{tabular}{c|c|c|c|c|c|c}
\hline \hline Day & $3-7 \mathrm{keV}$ & $7-30 \mathrm{keV}$ & $30-80 \mathrm{keV}$ & $0.2-0.4 \mathrm{TeV}$ & $0.4-0.8 \mathrm{TeV}$ & $>0.8 \mathrm{TeV}$ \\
\hline 11 April 2013 & 0.2657 & 0.1670 & 0.02437 & 3.872 & 1.312 & 0.4375 \\
12 April 2013 & 0.7881 & 0.6725 & 0.1291 & 8.431 & 3.683 & 1.834 \\
13 April 2013 & 1.119 & 0.9506 & 0.1737 & 10.23 & 4.889 & 2.713 \\
14 April 2013 & 0.4043 & 0.2806 & 0.04366 & 5.758 & 2.286 & 0.9662 \\
15 April 2013 & 1.021 & 1.194 & 0.3437 & 7.373 & 3.493 & 2.326 \\
16 April 2013 & 0.4054 & 0.3498 & 0.07931 & 4.211 & 1.561 & 0.6639 \\
17 April 2013 & 0.3190 & 0.3397 & 0.1042 & 2.706 & 0.9845 & 0.37643 \\
18 April 2013 & 0.08387 & 0.05741 & 0.007850 & 2.053 & 0.5959 & 0.2168 \\
19 April 2013 & 0.07172 & 0.03977 & 0.005220 & 1.175 & 0.3813 & 0.09668 \\
\hline
\end{tabular}

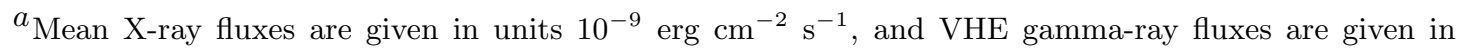
$10^{-10} \mathrm{ph} \mathrm{cm}^{-2} \mathrm{~s}^{-1}$.

\section{FLUX VARIATIONS ON MULTI-HOURS AND SUB-HOURS TIMESCALES}

This section reports the quantification of the X-ray and VHE gamma-ray multi-band flux variability during single nights using the templates defined by Eq. 3 in Section 4.2. The best-fit results obtained with the light curves from April 11-14 are reported in Table 7, while the results obtained for April 16 are reported in Table 8. Below we provide a brief description of the used strategy and the obtained results for each of the nights.

\section{C.1. 11 April 2013}

There are no flares visible in any of the VHE or X-ray bands. The light curves are not consistent with constant flux, and are fit with a linear model, i.e. the Slow component. The results are shown in Fig. 13. For all the $\mathrm{X}$-ray and VHE gamma-ray bands from these 10-hour long light curves, the slopes are all consistent within the error bars.

\section{C.2. 12 April 2013}

There is a clear flare visible in all X-ray bands around 2 hours past midnight; but unfortunately there is a gap in the VHE coverage for that time interval. Therefore VHE light curves are fit with a linear function (Slow component) only. The results are shown in Fig. 14. All fits are performed only up to $7.7 \mathrm{hrs}$ past midnight. There is flickering in VHE bands, with light curves inconsistent with constant flux, and simple linear model or Slow+Fast component model from section 4.2 does not describe data well either. Fits with Slow component give low slope values, consistent with zero for bands above $0.4 \mathrm{TeV}$.

Slopes of the Slow component decrease with increasing energy in X-ray bands as well, while flare amplitude increases with energy.

\section{C.3. 13 April 2013}

There is a flare visible in the two higher VHE bands, with the amplitude and the width of the flare both approximately halved in the $0.4-0.8 \mathrm{TeV}$ band w.r.t. the $>0.8 \mathrm{TeV}$ band. In the lowest VHE band, there are flux measurements above the Slow component at the location of the flare, but no satisfactory Slow + Fast model fit (section 4.2) could be obtained. There is a $\sim 2$-hour gap between the MAGIC and VERITAS data, and the fit is performed only with MAGIC data point, denoted by using gray instead of green color for VERITAS data in Fig. 15.

There is no X-ray data covering the time of the VHE flare. On the other hand, there is a small-amplitude flare around $2.5 \mathrm{hrs}$ earlier visible in X-ray bands, without obvious counterpart in VHE bands. Being relatively weak mini-flare, with the highest flux measured at the same time in all three X-ray bands, the fit is performed with Slow+Fast model, but with flare time (location) fixed to the time of the highest flux measurement, at midnight -0.24 hrs.

\section{C.4. 14 April 2013}

There is no obvious flare component in the VHE bands, therefore VHE bands are fit with Slow compo- 
nents only (Fig. 16). In X-ray bands the flux goes down, then increases in a broad flare visible in two lower X-ray bands, while no such behavior is seen in VHE bands. Flare amplitudes in X-ray bands are consistent within the error bars, but there are some data missing during the time covered by the Fast component, therefore no definite conclusions about the flaring component can be made.

The second part of the light curves (about 1 hour after midinght) has more simple structure, with a significant decrease in flux. Slow component slopes in all bands are similar.

\section{C.5. 16 April 2013}

There are no characteristic mini-flares in the 10-hour long light curve from April 16. Instead, there is a prominent rise and then fall light curves from all energy bands. This behavior cannot be fit by the Slow+Fast model from section 4.2. Because of that, we modify the model to exclude the Slow component, and only have a broad 'bump' with different rise and fall times:

$$
F_{B}(t)=\frac{2}{2^{-\frac{t-t_{0}}{t_{\mathrm{rise}}}}+2^{\frac{t-t_{0}}{t_{\text {fall }}}}} \cdot A
$$

The fit is performed to all three X-ray bands simultaneously, giving a unique rise time $t_{\text {rise }}=1.38 \pm 0.23 \mathrm{hrs}$, fall time $t_{\text {fall }}=4.96 \pm 0.47 \mathrm{hrs}$, and break time $t_{0}=-0.42 \pm 0.34 \mathrm{hrs}$ from midnight, with

$\chi^{2} /$ d.o.f. $=582 / 57$. Only flare amplitude is allowed to vary in different bands, with results reported in Table 8. After fitting to X-ray data, the function shape is fit to each VHE band, again allowing only the flare amplitude to vary. The results, along with the $\chi^{2}$ values for each band are reported in Table 8 . 

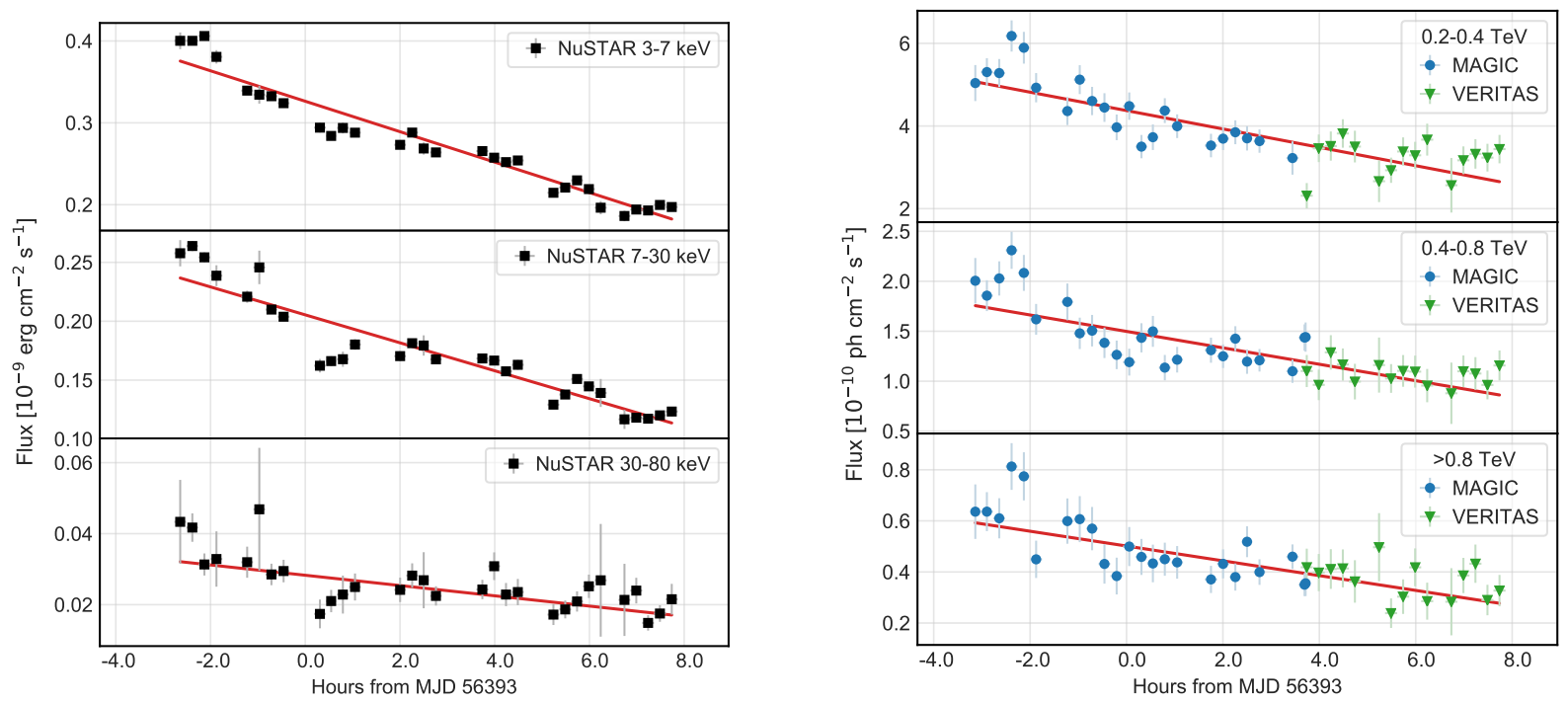

Figure 13. Light curves from 2013 April 11 in three X-ray bands (left panel) and three VHE gamma-ray bands (right panel). The red curve is the resulting fit with the function defined by Eq. 3, whose model parameters are reported in Table 7.
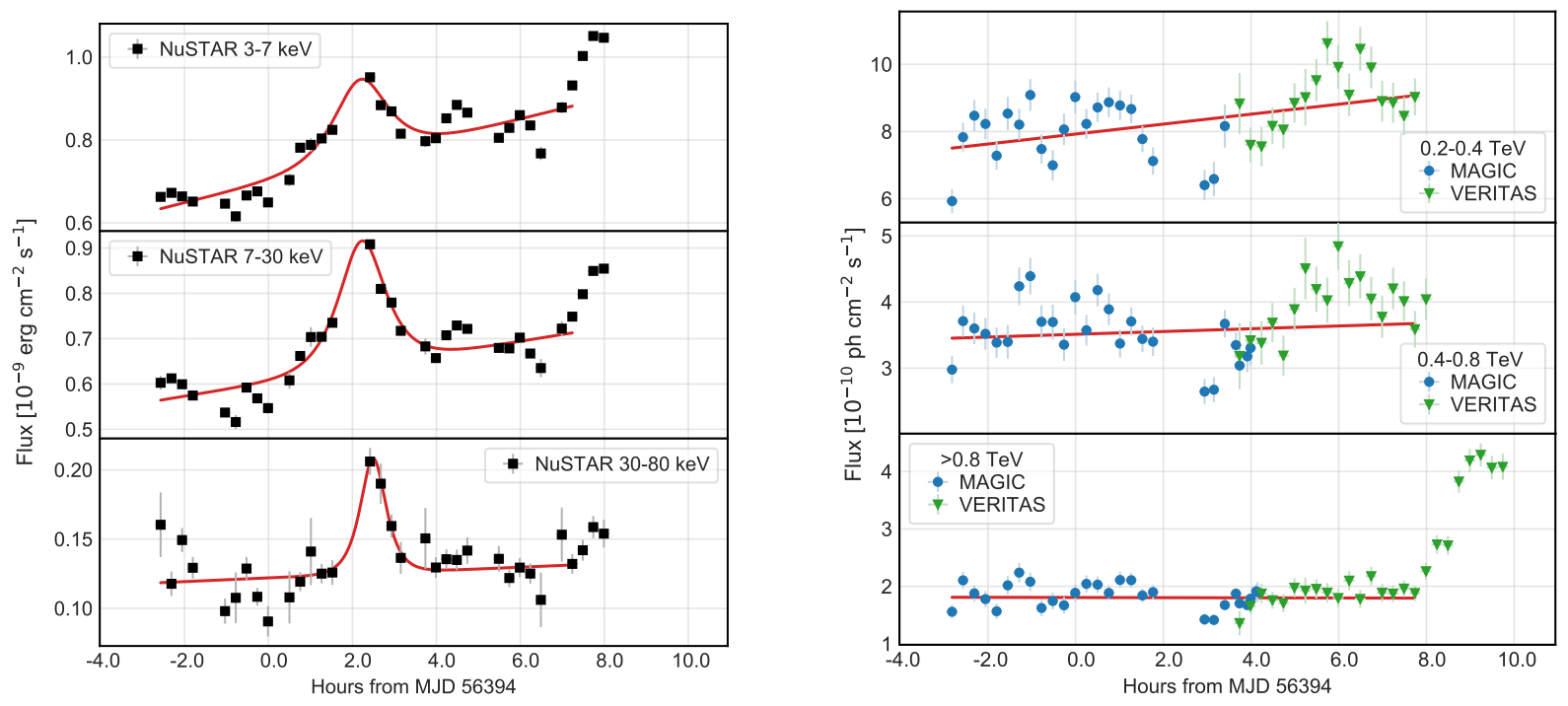

Figure 14. Light curves from 2013 April 12 in three X-ray bands (left panel) and three VHE gamma-ray bands (right panel). The red curve is the resulting fit with the function defined by Eq. 3, whose model parameters are reported in Table 7. 

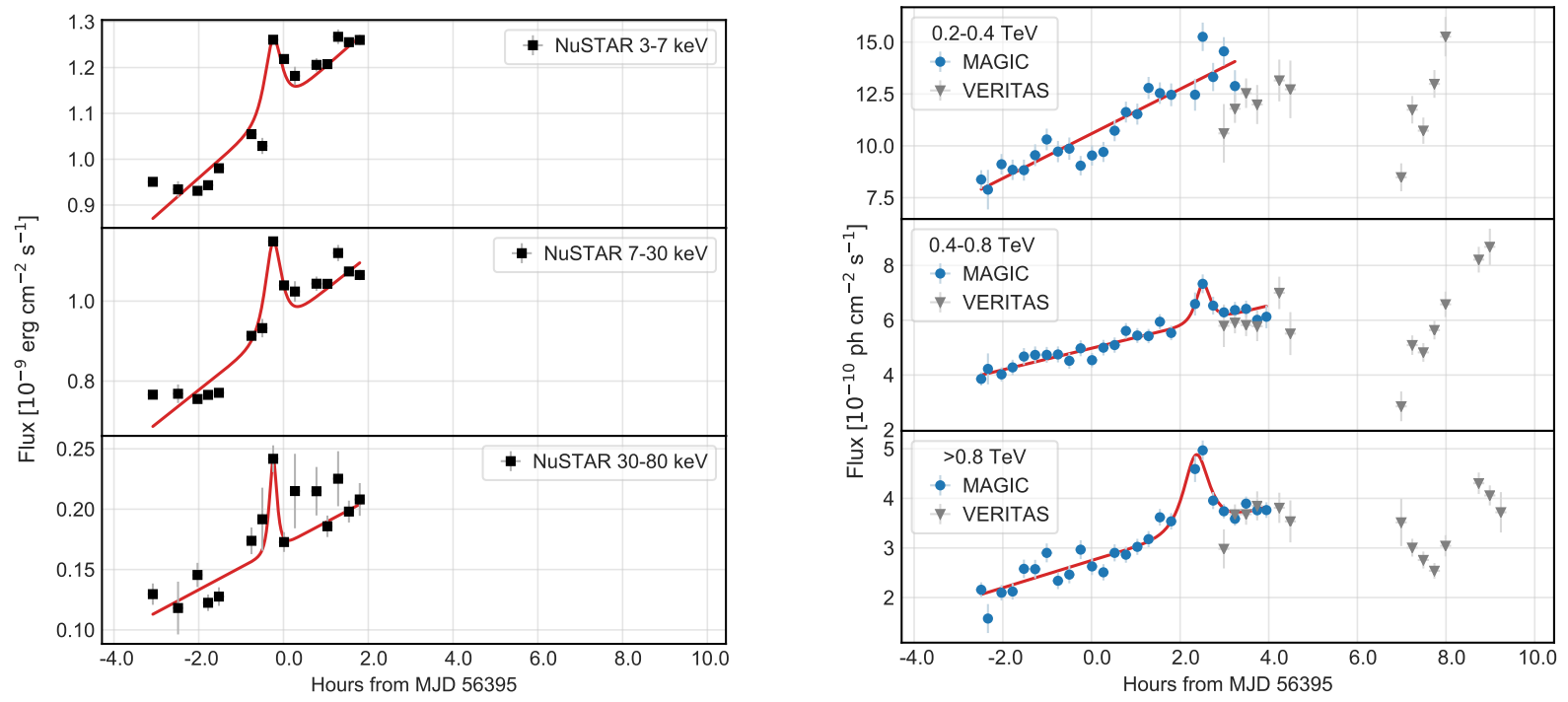

Figure 15. Light curves from 2013 April 13 in three X-ray bands (left panel) and three VHE gamma-ray bands (right panel). The red curve is the resulting fit with the function defined by Eq. 3, whose model parameters are reported in Table 7.
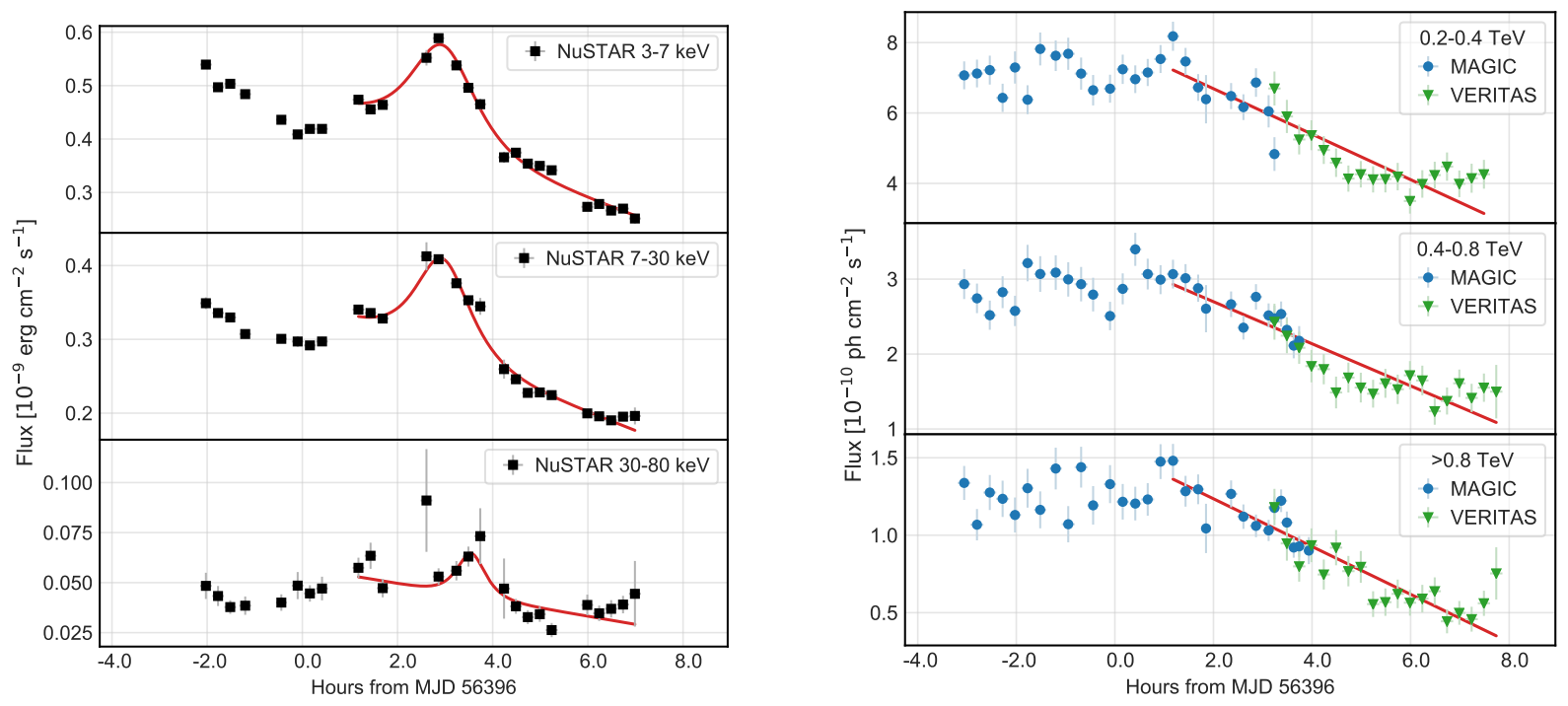

Figure 16. Light curves from 2013 April 14 in three X-ray bands (left panel) and three VHE gamma-ray bands (right panel). The red curve is the resulting fit with the function defined by Eq. 3, whose model parameters are reported in Table 7. 

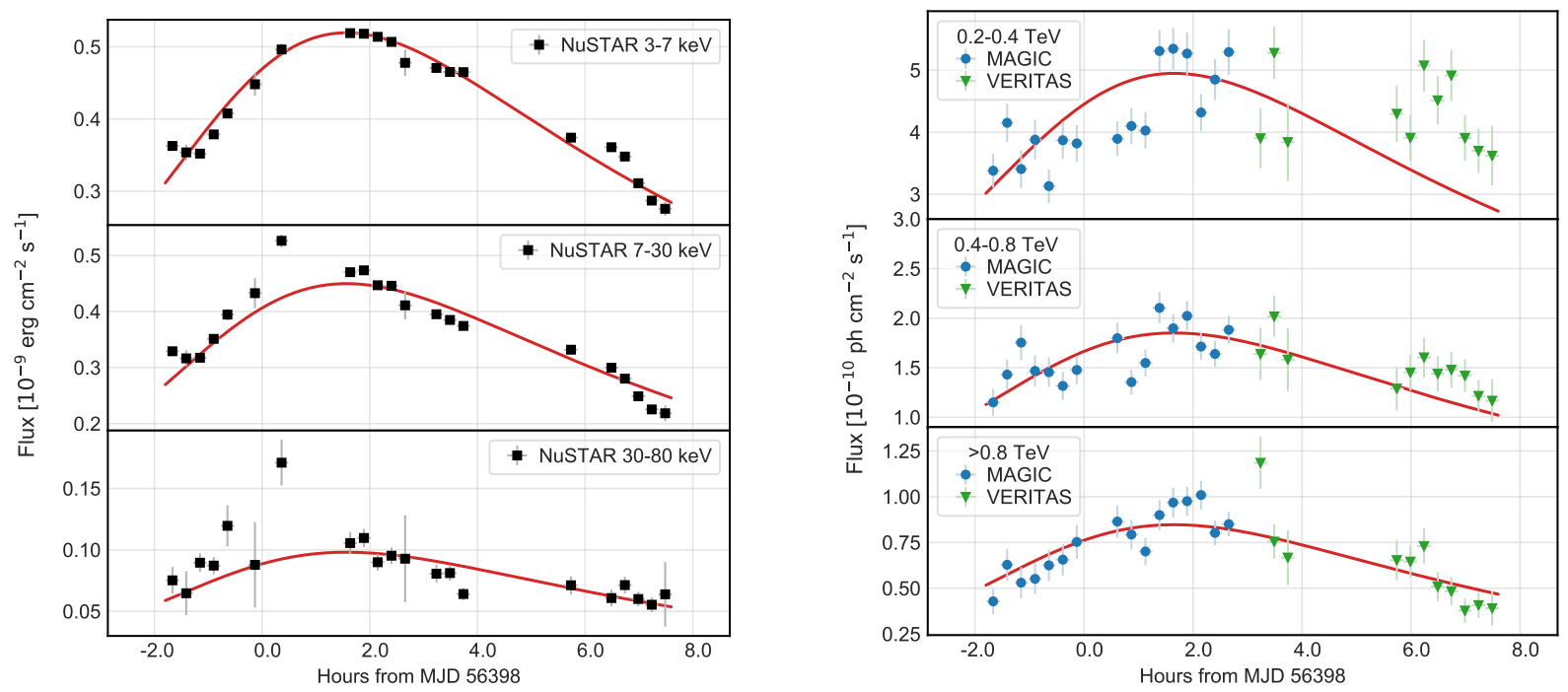

Figure 17. Light curves from 2013 April 16 in three X-ray bands (left panel) and three VHE gamma-ray bands (right panel). The red curve is the resulting fit with the function defined by Eq. C1, whose model parameters are reported in Table 8. 
Table 7. Parameters resulting from the fit with Eq. 3 to the X-ray and VHE multi-band light curves for 2013 April 11-14.

\begin{tabular}{|c|c|c|c|c|c|c|}
\hline Band & Offset ${ }^{\mathrm{a}}$ & $\begin{array}{l}\text { Slope } \\
{\left[\mathrm{h}^{-1}\right]}\end{array}$ & $\begin{array}{c}\text { Flare } \\
\text { Amplitude } A\end{array}$ & $\begin{array}{c}\text { Flare } \\
\text { flux-doubling time } \mathrm{e}^{\mathrm{b}}[\mathrm{h}]\end{array}$ & $\begin{array}{l}\text { Flare } \\
t_{0}[\mathrm{~h}]\end{array}$ & $\chi^{2} /$ d.o.f \\
\hline \multicolumn{7}{|c|}{11 April 2013} \\
\hline $3-7 \mathrm{keV}$ & $0.326 \pm 0.004$ & $-0.057 \pm 0.002$ & - & - & - & $384 / 28$ \\
\hline $7-30 \mathrm{keV}$ & $0.205 \pm 0.004$ & $-0.058 \pm 0.003$ & - & - & - & $320 / 28$ \\
\hline $30-80 \mathrm{keV}$ & $0.028 \pm 0.001$ & $-0.051 \pm 0.007$ & - & - & - & $37.7 / 28$ \\
\hline $0.2-0.4 \mathrm{TeV}$ & $4.37 \pm 0.10$ & $-0.051 \pm 0.006$ & - & - & - & $74.7 / 35$ \\
\hline $0.4-0.8 \mathrm{TeV}$ & $1.50 \pm 0.04$ & $-0.055 \pm 0.006$ & - & - & - & $58.1 / 37$ \\
\hline$>0.8 \mathrm{TeV}$ & $0.50 \pm 0.02$ & $-0.058 \pm 0.007$ & - & - & - & $44.0 / 37$ \\
\hline \multicolumn{7}{|c|}{12 April 2013} \\
\hline 3-7 keV & $0.70 \pm 0.01$ & $0.041 \pm 0.003$ & $0.26 \pm 0.07$ & $0.34 \pm 0.09$ & $2.2 \pm 0.1$ & $764 / 28$ \\
\hline $7-30 \mathrm{keV}$ & $0.604 \pm 0.009$ & $0.029 \pm 0.004$ & $0.44 \pm 0.08$ & $0.32 \pm 0.05$ & $2.21 \pm 0.08$ & $449 / 28$ \\
\hline $30-80 \mathrm{keV}$ & $0.122 \pm 0.003$ & $0.013 \pm 0.006$ & $0.66 \pm 0.12$ & $0.18 \pm 0.07$ & $2.5 \pm 0.1$ & $53.9 / 28$ \\
\hline $0.2-0.4 \mathrm{TeV}$ & $7.9 \pm 0.2$ & $0.019 \pm 0.006$ & - & - & - & $131 / 37$ \\
\hline $0.4-0.8 \mathrm{TeV}$ & $3.51 \pm 0.09$ & $0.006 \pm 0.007$ & - & - & - & $146 / 42$ \\
\hline$>0.8 \mathrm{TeV}$ & $1.81 \pm 0.04$ & $-0.001 \pm 0.006$ & - & - & - & $122 / 51$ \\
\hline \multicolumn{7}{|c|}{13 April 2013} \\
\hline $3-7 \mathrm{keV}$ & $1.12 \pm 0.01$ & $0.15 \pm 0.04$ & $0.12 \pm 0.04$ & $0.073 \pm 0.007$ & - & $237 / 11$ \\
\hline $7-30 \mathrm{keV}$ & $0.94 \pm 0.02$ & $0.24 \pm 0.05$ & $0.11 \pm 0.03$ & $0.089 \pm 0.008$ & - & $165 / 11$ \\
\hline $30-80 \mathrm{keV}$ & $0.17 \pm 0.01$ & $0.46 \pm 0.11$ & $0.053 \pm 0.039$ & $0.11 \pm 0.02$ & - & $25.3 / 11$ \\
\hline $0.2-0.4 \mathrm{TeV}$ & $10.6 \pm 0.2$ & $0.10 \pm 0.01$ & - & - & - & $40.2 / 21$ \\
\hline $0.4-0.8 \mathrm{TeV}$ & $4.97 \pm 0.05$ & $0.079 \pm 0.006$ & $0.23 \pm 0.05$ & $0.098 \pm 0.029$ & $2.52 \pm 0.05$ & $12.6 / 21$ \\
\hline$>0.8 \mathrm{TeV}$ & $2.74 \pm 0.05$ & $0.10 \pm 0.01$ & $0.44 \pm 0.08$ & $0.17 \pm 0.04$ & $2.36 \pm 0.07$ & $33.3 / 21$ \\
\hline \multicolumn{7}{|c|}{14 April 2013} \\
\hline $3-7 \mathrm{keV}$ & $0.49 \pm 0.03$ & $-0.068 \pm 0.005$ & $0.48 \pm 0.06$ & $0.42 \pm 0.09$ & $2.95 \pm 0.09$ & $158 / 21$ \\
\hline $7-30 \mathrm{keV}$ & $0.35 \pm 0.02$ & $-0.071 \pm 0.004$ & $0.47 \pm 0.05$ & $0.35 \pm 0.08$ & $2.95 \pm 0.09$ & $74.6 / 21$ \\
\hline $30-80 \mathrm{keV}$ & $0.058 \pm 0.006$ & $-0.071 \pm 0.013$ & $0.50 \pm 0.23$ & $0.18 \pm 0.13$ & $3.5 \pm 0.3$ & $50.8 / 21$ \\
\hline $0.2-0.4 \mathrm{TeV}$ & $8.0 \pm 0.3$ & $-0.081 \pm 0.005$ & - & - & - & $75.8 / 41$ \\
\hline $0.4-0.8 \mathrm{TeV}$ & $3.3 \pm 0.1$ & $-0.086 \pm 0.004$ & - & - & - & $60.4 / 46$ \\
\hline$>0.8 \mathrm{TeV}$ & $1.54 \pm 0.05$ & $-0.100 \pm 0.004$ & - & - & - & $61.5 / 47$ \\
\hline
\end{tabular}

${ }^{a}$ For VHE bands in $10^{-10} \mathrm{ph} \mathrm{cm}^{-2} \mathrm{~s}^{-1}$, for X-ray bands in $10^{-9} \mathrm{erg} \mathrm{cm}^{-2} \mathrm{~s}^{-1}$.

${ }^{b}$ Parameters $t_{\text {rise }}$ and $t_{\text {fall }}$ in Eq. 3 are set to be equal, and correspond to the flare flux-doubling time in this Table. 
Table 8. Parameters resulting from the fit with Eq. (C1) to the X-ray and VHE multi-band light curves from 2013 April 16. The three X-ray bands are fit with a single function, yielding $t_{\text {rise }}=1.4 \pm 0.2 \mathrm{hrs}, t_{\text {fall }}=5.0 \pm 0.5 \mathrm{hrs}$, break time $t_{0}=-0.42 \pm 0.34$ hrs from midnight, with $\chi^{2} /$ d.o.f. $=582 / 57$.

\begin{tabular}{r|c|r}
\hline Band & Flare Amplitude $A^{\mathrm{a}}$ & $\chi^{2} /$ d.o.f. \\
\hline $3-7 \mathrm{keV}$ & $0.44 \pm 0.02$ & - \\
$7-30 \mathrm{keV}$ & $0.38 \pm 0.02$ & - \\
$30-80 \mathrm{keV}$ & $0.083 \pm 0.007$ & - \\
$0.2-0.4 \mathrm{TeV}$ & $4.2 \pm 0.2$ & $140 / 26$ \\
$0.4-0.8 \mathrm{TeV}$ & $1.57 \pm 0.05$ & $55 / 26$ \\
$>0.8 \mathrm{TeV}$ & $0.72 \pm 0.02$ & $39 / 26$ \\
\hline
\end{tabular}

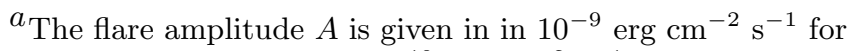
the X-ray bands, and $10^{-10} \mathrm{ph} \mathrm{cm}^{-2} \mathrm{~s}^{-1}$ for the VHE gamma-ray bands. 


\section{SINGLE-DAY FLUX-FLUX CORRELATIONS}

In this section we report the quantification of the VHE vs X-ray correlations for single-night data. In particular, we report the Pearson correlation coefficients and related significances, the values of the DCF, and the slopes and the $\chi^{2}$ values from the linear fits to the $\log F_{\mathrm{VHE}}-\log F_{\mathrm{X}-\text { ray }}$. These numbers are complementary to those reported in Table 4, which report the same information for the nine-day data set (instead of singlenight data sets).

Table 9. Single-night correlation coefficients and fit slopes for 2019 April 11-16. The statistical and systematic contributions to the total uncertainty are reported separately, and in this order.

\begin{tabular}{|c|c|c|c|c|c|c|}
\hline VHE band & X-ray band & Pearson coeff. & $N \sigma$ (Pearson) & $\mathrm{DCF}$ & Linear fit slope & $\chi^{2} /$ d.o.f \\
\hline \multicolumn{7}{|c|}{11 April 2013} \\
\hline \multirow[t]{3}{*}{$0.2-0.4 \mathrm{TeV}$} & $3-7 \mathrm{keV}$ & $0.83_{-0.07}^{+0.05} \pm 0.02$ & 6.1 & $0.90 \pm 0.21 \pm 0.007$ & $0.86 \pm 0.09 \pm 0.05$ & $43 / 28$ \\
\hline & $7-30 \mathrm{keV}$ & $0.84_{-0.07}^{+0.05} \pm 0.01$ & 6.4 & $0.92 \pm 0.22 \pm 0.003$ & $0.83 \pm 0.08 \pm 0.05$ & $37 / 28$ \\
\hline & $30-80 \mathrm{keV}$ & $0.74_{-0.10}^{+0.08} \pm 0.002$ & 4.9 & $1.35 \pm 0.37 \pm 0.02$ & $1.02 \pm 0.15 \pm 0.07$ & $28 / 28$ \\
\hline \multirow[t]{3}{*}{$0.4-0.8 \mathrm{TeV}$} & $3-7 \mathrm{keV}$ & $0.89_{-0.05}^{+0.03} \pm 0.04$ & 7.3 & $1.06 \pm 0.26 \pm 0.02$ & $1.01 \pm 0.09 \pm 0.11$ & $26 / 28$ \\
\hline & $7-30 \mathrm{keV}$ & $0.86_{-0.06}^{+0.04} \pm 0.03$ & 6.7 & $1.03 \pm 0.26 \pm 0.03$ & $0.95 \pm 0.09 \pm 0.10$ & $30 / 28$ \\
\hline & $30-80 \mathrm{keV}$ & $0.64_{-0.13}^{+0.10} \pm 0.01$ & 4.0 & $1.29 \pm 0.45 \pm 0.06$ & $1.08 \pm 0.15 \pm 0.13$ & $25 / 28$ \\
\hline \multirow[t]{3}{*}{$>0.8 \mathrm{TeV}$} & $3-7 \mathrm{keV}$ & $0.79_{-0.08}^{+0.06} \pm 0.04$ & 5.6 & $1.04 \pm 0.28 \pm 0.01$ & $1.01 \pm 0.13 \pm 0.10$ & $28 / 28$ \\
\hline & $7-30 \mathrm{keV}$ & $0.78_{-0.09}^{+0.07} \pm 0.04$ & 5.4 & $1.03 \pm 0.28 \pm 0.02$ & $0.96 \pm 0.12 \pm 0.09$ & $27 / 28$ \\
\hline & $30-80 \mathrm{keV}$ & $0.61_{-0.14}^{+0.11} \pm 0.02$ & 3.7 & $1.35 \pm 0.47 \pm 0.05$ & $1.2 \pm 0.2 \pm 0.1$ & $25 / 28$ \\
\hline \multicolumn{7}{|c|}{12 April 2013} \\
\hline \multirow[t]{3}{*}{$0.2-0.4 \mathrm{TeV}$} & $3-7 \mathrm{keV}$ & $0.26_{-0.19}^{+0.17} \pm 0.10$ & 1.4 & $0.31 \pm 0.17 \pm 0.11$ & $0.21 \pm 0.14 \pm 0.09$ & $78 / 27$ \\
\hline & $7-30 \mathrm{keV}$ & $0.16_{-0.19}^{+0.18} \pm 0.09$ & 0.8 & $0.19 \pm 0.19 \pm 0.10$ & $0.15 \pm 0.09 \pm 0.09$ & $82 / 27$ \\
\hline & $30-80 \mathrm{keV}$ & $-0.19_{-0.18}^{+0.19} \pm 0.07$ & 1.0 & $-0.31 \pm 0.24 \pm 0.12$ & $-1.03 \pm 0.39 \pm 14$ & $66 / 27$ \\
\hline \multirow[t]{3}{*}{$0.4-0.8 \mathrm{TeV}$} & $3-7 \mathrm{keV}$ & $0.05_{-0.19}^{+0.18} \pm 0.19$ & 0.3 & $0.06 \pm 0.16 \pm 0.23$ & $0.02 \pm 0.16 \pm 0.18$ & $90 / 30$ \\
\hline & $7-30 \mathrm{keV}$ & $-0.07_{-0.18}^{+0.19} \pm 0.17$ & 0.4 & $-0.09 \pm 0.18 \pm 0.21$ & $-0.12 \pm 0.18 \pm 0.18$ & $89 / 30$ \\
\hline & $30-80 \mathrm{keV}$ & $-0.37_{-0.15}^{+0.17} \pm 0.12$ & 2.1 & $-0.65 \pm 0.27 \pm 0.23$ & $-0.76 \pm 0.23 \pm 20$ & $59 / 30$ \\
\hline \multirow[t]{3}{*}{$>0.8 \mathrm{TeV}$} & $3-7 \mathrm{keV}$ & $0.19_{-0.18}^{+0.17} \pm 0.19$ & 1.0 & $0.27 \pm 0.25 \pm 0.24$ & $0.15 \pm 0.14 \pm 0.17$ & $61 / 30$ \\
\hline & $7-30 \mathrm{keV}$ & $0.18_{-0.18}^{+0.17} \pm 0.16$ & 1.0 & $0.26 \pm 0.27 \pm 0.21$ & $0.18 \pm 0.16 \pm 0.17$ & $61 / 30$ \\
\hline & $30-80 \mathrm{keV}$ & $-0.04_{-0.18}^{+0.19} \pm 0.09$ & 0.2 & $-0.07 \pm 0.36 \pm 0.17$ & $0.48 \pm 0.20 \pm 0.79$ & $61 / 30$ \\
\hline \multicolumn{7}{|c|}{13 April $2013^{*}$} \\
\hline \multirow[t]{3}{*}{$0.2-0.4 \mathrm{TeV}$} & $3-7 \mathrm{keV}$ & $0.74_{-0.17}^{+0.11}$ & 3.2 & $0.73 \pm 0.24$ & $0.98 \pm 0.24$ & $52 / 12$ \\
\hline & $7-30 \mathrm{keV}$ & $0.70_{-0.19}^{+0.13}$ & 2.9 & $0.69 \pm 0.24$ & $0.75 \pm 0.21$ & $60 / 12$ \\
\hline & $30-80 \mathrm{keV}$ & $0.65_{-0.21}^{+0.14}$ & 2.6 & $0.70 \pm 0.28$ & $0.58 \pm 0.17$ & $49 / 12$ \\
\hline \multirow[t]{3}{*}{$0.4-0.8 \mathrm{TeV}$} & $3-7 \mathrm{keV}$ & $0.86_{-0.11}^{+0.06}$ & 4.2 & $0.89 \pm 0.25$ & $0.94 \pm 0.16$ & $19 / 12$ \\
\hline & $7-30 \mathrm{keV}$ & $0.81_{-0.13}^{+0.08}$ & 3.7 & $0.84 \pm 0.25$ & $0.72 \pm 0.15$ & $24 / 12$ \\
\hline & $30-80 \mathrm{keV}$ & $0.76_{-0.16}^{+0.10}$ & 3.3 & $0.85 \pm 0.28$ & $0.50 \pm 0.12$ & $23 / 12$ \\
\hline \multirow[t]{2}{*}{$>0.8 \mathrm{TeV}$} & $3-7 \mathrm{keV}$ & $0.873_{-0.094}^{+0.056}$ & 4.5 & $0.87 \pm 0.22$ & $1.40 \pm 0.23$ & $28 / 12$ \\
\hline & 7-30 keV & $0.81_{-0.13}^{+0.08}$ & 3.8 & $0.81 \pm 0.22$ & $1.07 \pm 0.23$ & $41 / 12$ \\
\hline
\end{tabular}


Table 9 (continued)

\begin{tabular}{|c|c|c|c|c|c|c|}
\hline VHE band & X-ray band & Pearson coeff. & $N \sigma$ (Pearson) & $\mathrm{DCF}$ & Linear fit slope & $\chi^{2} /$ d.o.f \\
\hline & $30-80 \mathrm{keV}$ & $0.70_{-0.19}^{+0.12}$ & 2.9 & $0.76 \pm 0.24$ & $0.85 \pm 0.21$ & $36 / 12$ \\
\hline \multicolumn{7}{|c|}{14 April 2013} \\
\hline \multirow[t]{3}{*}{$0.2-0.4 \mathrm{TeV}$} & $3-7 \mathrm{keV}$ & $0.79_{-0.09}^{+0.07} \pm 0.003$ & 5.2 & $0.79 \pm 0.17 \pm 0.002$ & $0.82 \pm 0.15 \pm 0.05$ & $130 / 25$ \\
\hline & $7-30 \mathrm{keV}$ & $0.78_{-0.09}^{+0.07} \pm 0.003$ & 5.2 & $0.79 \pm 0.16 \pm 0.002$ & $0.87 \pm 0.15 \pm 0.06$ & $126 / 25$ \\
\hline & $30-80 \mathrm{keV}$ & $0.45_{-0.18}^{+0.15} \pm 0.002$ & 2.4 & $0.55 \pm 0.20 \pm 0.006$ & $1.5 \pm 0.4 \pm 0.1$ & $76 / 25$ \\
\hline \multirow[t]{3}{*}{$0.4-0.8 \mathrm{TeV}$} & $3-7 \mathrm{keV}$ & $0.78_{-0.09}^{+0.07} \pm 0.006$ & 5.3 & $0.79 \pm 0.17 \pm 0.006$ & $0.79 \pm 0.18 \pm 0.11$ & $138 / 27$ \\
\hline & $7-30 \mathrm{keV}$ & $0.78_{-0.09}^{+0.07} \pm 0.009$ & 5.3 & $0.80 \pm 0.17 \pm 0.006$ & $0.85 \pm 0.19 \pm 0.12$ & $138 / 27$ \\
\hline & $30-80 \mathrm{keV}$ & $0.41_{-0.17}^{+0.15} \pm 0.007$ & 2.2 & $0.51 \pm 0.19 \pm 0.002$ & $1.7 \pm 0.5 \pm 0.3$ & $94 / 27$ \\
\hline \multirow[t]{3}{*}{$>0.8 \mathrm{TeV}$} & $3-7 \mathrm{keV}$ & $0.83_{-0.07}^{+0.05} \pm 0.003$ & 6.1 & $0.85 \pm 0.21 \pm 0.007$ & $0.94 \pm 0.18 \pm 0.10$ & $107 / 27$ \\
\hline & $7-30 \mathrm{keV}$ & $0.81_{-0.08}^{+0.06} \pm 0.006$ & 5.7 & $0.83 \pm 0.19 \pm 0.003$ & $0.93 \pm 0.19 \pm 0.11$ & $112 / 27$ \\
\hline & $30-80 \mathrm{keV}$ & $0.42_{-0.17}^{+0.15} \pm 0.007$ & 2.3 & $0.51 \pm 0.20 \pm 0.003$ & $1.6 \pm 0.4 \pm 0.2$ & $86 / 27$ \\
\hline \multicolumn{7}{|c|}{15 April 2013} \\
\hline \multirow[t]{3}{*}{$0.2-0.4 \mathrm{TeV}$} & $3-7 \mathrm{keV}$ & $0.77_{-0.09}^{+0.07} \pm 0.04$ & 5.3 & $0.82 \pm 0.20 \pm 0.04$ & $0.40 \pm 0.05 \pm 0.03$ & $68 / 28$ \\
\hline & $7-30 \mathrm{keV}$ & $0.75_{-0.10}^{+0.07} \pm 0.05$ & 5.1 & $0.81 \pm 0.19 \pm 0.04$ & $0.33 \pm 0.05 \pm 0.02$ & $72 / 28$ \\
\hline & $30-80 \mathrm{keV}$ & $0.71_{-0.11}^{+0.08} \pm 0.05$ & 4.6 & $0.77 \pm 0.18 \pm 0.05$ & $0.29 \pm 0.05 \pm 0.02$ & $80 / 28$ \\
\hline \multirow[t]{3}{*}{$0.4-0.8 \mathrm{TeV}$} & $3-7 \mathrm{keV}$ & $0.80_{-0.08}^{+0.06} \pm 0.08$ & 5.8 & $0.86 \pm 0.19 \pm 0.08$ & $0.52 \pm 0.07 \pm 0.04$ & $80 / 29$ \\
\hline & $7-30 \mathrm{keV}$ & $0.78_{-0.09}^{+0.06} \pm 0.08$ & 5.5 & $0.84 \pm 0.19 \pm 0.08$ & $0.41 \pm 0.06 \pm 0.03$ & $88 / 29$ \\
\hline & $30-80 \mathrm{keV}$ & $0.73_{-0.10}^{+0.08} \pm 0.09$ & 5.0 & $0.81 \pm 0.18 \pm 0.09$ & $0.36 \pm 0.06 \pm 0.03$ & $93 / 29$ \\
\hline \multirow[t]{3}{*}{$>0.8 \mathrm{TeV}$} & $3-7 \mathrm{keV}$ & $0.95 \pm 0.02 \pm 0.02$ & 9.5 & $0.94 \pm 0.19 \pm 0.02$ & $0.91 \pm 0.07 \pm 0.05$ & $77 / 29$ \\
\hline & $7-30 \mathrm{keV}$ & $0.94_{-0.03}^{+0.02} \pm 0.02$ & 9.1 & $0.94 \pm 0.19 \pm 0.02$ & $0.74 \pm 0.06 \pm 0.04$ & $87 / 29$ \\
\hline & $30-80 \mathrm{keV}$ & $0.88_{-0.05}^{+0.04} \pm 0.03$ & 7.3 & $0.90 \pm 0.15 \pm 0.02$ & $0.63 \pm 0.06 \pm 0.03$ & $90 / 29$ \\
\hline \multicolumn{7}{|c|}{16 April 2013} \\
\hline \multirow[t]{3}{*}{$0.2-0.4 \mathrm{TeV}$} & $3-7 \mathrm{keV}$ & $0.53_{-0.20}^{+0.15} \pm 0.05$ & 2.5 & $0.62 \pm 0.22 \pm 0.05$ & $0.56 \pm 0.16 \pm 0.04$ & $51 / 18$ \\
\hline & $7-30 \mathrm{keV}$ & $0.42_{-0.22}^{+0.18} \pm 0.06$ & 1.8 & $0.49 \pm 0.24 \pm 0.07$ & $0.39 \pm 0.16 \pm 0.05$ & $62 / 18$ \\
\hline & $30-80 \mathrm{keV}$ & $0.21_{-0.24}^{+0.22} \pm 0.08$ & 0.9 & $0.47 \pm 0.61 \pm 0.17$ & $0.77 \pm 0.26 \pm 0.02$ & $51 / 18$ \\
\hline \multirow[t]{3}{*}{$0.4-0.8 \mathrm{TeV}$} & $3-7 \mathrm{keV}$ & $0.78_{-0.11}^{+0.08} \pm 0.06$ & 4.3 & $1.1 \pm 0.3 \pm 0.07$ & $0.67 \pm 0.13 \pm 0.09$ & $19 / 18$ \\
\hline & $7-30 \mathrm{keV}$ & $0.72_{-0.14}^{+0.10} \pm 0.09$ & 3.7 & $1.0 \pm 0.3 \pm 0.09$ & $0.55 \pm 0.13 \pm 0.10$ & $23 / 18$ \\
\hline & $30-80 \mathrm{keV}$ & $0.61_{-0.17}^{+0.13} \pm 0.12$ & 2.9 & $1.6 \pm 0.5 \pm 0.3$ & $0.68 \pm 0.15 \pm 0.11$ & $18 / 18$ \\
\hline \multirow[t]{3}{*}{$>0.8 \mathrm{TeV}$} & $3-7 \mathrm{keV}$ & $0.92_{-0.05}^{+0.03} \pm 0.02$ & 6.5 & $0.97 \pm 0.21 \pm 0.01$ & $1.6 \pm 0.2 \pm 0.09$ & $19 / 18$ \\
\hline & $7-30 \mathrm{keV}$ & $0.88_{-0.07}^{+0.04} \pm 0.03$ & 5.8 & $0.94 \pm 0.23 \pm 0.03$ & $1.4 \pm 0.2 \pm 0.1$ & $27 / 18$ \\
\hline & $30-80 \mathrm{keV}$ & $0.64_{-0.17}^{+0.12} \pm 0.05$ & 3.1 & $1.3 \pm 0.4 \pm 0.1$ & $1.8 \pm 0.4 \pm 0.06$ & $32 / 18$ \\
\hline
\end{tabular}

${ }^{*}$ There are no simultaneous NuSTAR and VERITAS data on April 13, and hence there is no systematic error associated to the usage of the flux-scale factors.

\section{REFERENCES}

Abdo, A. A., Ackermann, M., Ajello, M., et al. 2011, ApJ, 736,131

Abdollahi S., Acero F., Ackermann M., et al. 2019, ApJS, in press, arXiv:1902.10045
Abeysekara, A. U., Benbow, W., Bird, R., et al. 2020 ApJ, in press (to be updated with arXiv coordinates once out) 
Acero, F., Ackermann, M., Ajello, M., et al. 2015, ApJS, 218,23

Agudo, I., Thum, C., Molina, S., et al. 2018, MNRAS, 473, 1850

Agudo, I., Thum, C., Molina, S., et al. 2018, MNRAS, 474, 1427

Aharonian, F., Akhperjanian, A. G., Bazer-Bachi, A. R., et al. 2007, ApJL, 664, L71

Aharonian, F., Akhperjanian, A. G., Anton, G., et al. 2009, ApJL, 696, L150

Aleksić, J., Ansoldi, S., Antonelli, L. A., et al. 2014, A\&A, 572, A121

Aleksić, J., Ansoldi, S., Antonelli, L. A., et al. 2015, A\&A, 573, A50

Aleksić, J., Ansoldi, S., Antonelli, L. A., et al. 2015, A\&A, 576, A126

Aleksić, J., Ansoldi, S., Antonelli, L. A., et al. 2016, Astroparticle Physics, 72, 76.

Arnaud, K. A. 1996, Astronomical Data Analysis Software and Systems V, 17

Baloković, M., Furniss, A., Madejski, G., \& Harrison, F. 2013, The Astronomer's Telegram, 4974

Baloković, M., Paneque, D., Madejski, G., et al. 2016, ApJ, 819, 156

Benbow, W. 2017, 35th International Cosmic Ray Conference, 2017, Bexco, Busan, Korea

Błażejowski, M., Blaylock, G., Bond, I. H., et al. 2005, ApJ, 630, 130

Astropy Collaboration, Robitaille, T. P., Tollerud, E. J., et al. 2013, A\&A, 558, A33

Bertin, E., \& Arnouts, S. 1996, A\&AS, 117, 393

Carnerero, M. I., Raiteri, C. M., Villata, M., et al. 2017, MNRAS, 472, 3789

Chen, X., Pohl, M., Böttcher, M., Gao, S. 2016, MNRAS, 458,3260

Christie, I. M., Petropoulou, M., Sironi, L., \& Giannios, D. 2019, MNRAS, 482, 65

Corrales, L. 2015, ApJ, 805, 23

Cortina, J., \& Holder, J. 2013, The Astronomer's Telegram, 4976

Dimitrakoudis, S., Mastichiadis, A., Protheroe, R. J., Reimer, A. 2012, A\&A, 546, A120

Dondi, L., \& Ghisellini, G. 1995, MNRAS, 273, 583

Ferland, G. J., Porter, R. L., van Hoof, P. A. M., et al. 2013, RMxAA, 49, 137

Finke, J. D. 2019, ApJ, 870, 28

Finke, J. D., Dermer, C. D., \& Böttcher, M. 2008, ApJ, 686,181

Fossati, G., Buckley, J. H., Bond I. H et al. 2008, ApJ, 677,906
Gaidos, J. A., Akerlof, C. W., Biller, S., et al. 1996, Nature, 383,319

Georganopoulos, M., \& Kazanas, D. 2003, ApJL, 594, L27

Ghisellini, G., Tavecchio, F., Foschini, L., Ghirlanda, G., Maraschi, L., Celotti, A. 2010, MNRAS, 402, 1

Ghisellini, G., Tavecchio, F., \& Chiaberge, M. 2005, A\&A, 432,401

Giannios, D., Uzdensky, D. A., \& Begelman, M. C. 2009, MNRAS, 395, L29

Giannios, D. and Uzdensky, D. A. and Begelman, M. C., 2010, MNRAS, 402, 1649

Giannios, D., 2013, MNRAS, 431, 355

Giannios, D., \& Uzdensky, D., 2019, MNRAS, 484, 1378

Hanisch, R. J., \& Biemesderfer, C. D. 1989, BAAS, 21, 780

Harrison, F. A., Craig, W. W., Christensen, F. E., et al. 2013, ApJ, 770, 103

Hervet, O., Williams, D. A., Falcone, A. D., et al. 2019, ApJ, 877, 26

Katarzyński, K., Ghisellini, G., Tavecchio, F., et al. 2005, A\&A, 433, 479

Kirk, J. G., Rieger, F. M., Mastichiadis, A. 1998, A\&A, 333,452

Krawczynski, H. and Coppi, P. S. and Aharonian, F. 2002, MNRAS, 336, 721

Horan, D., Acciari, V. A., Bradbury, S. M., et al. 2009, ApJ, 695, 596

Lamport, L. 1994, LaTeX: A Document Preparation System, 2nd Edition (Boston, Addison-Wesley Professional)

Lico, R., Giroletti, M., Orienti, M., et al. 2012, A\&A, 545, A117

Lin, Y. C., Bertsch, D. L., Chiang, J., et al. 1992, ApJL, 401, L61

Lister, M. L., Homan, D. C., Hovatta, T., et al. 2019, ApJ, 874,43

Madhavan, A. S., \& for the VERITAS Collaboration 2013, arXiv:1307.7051

Madsen, K. K., Harrison, F. A., Markwardt, C. B., et al. 2015, ApJS, 220, 8

Madsen, K. K., Beardmore, A. P., Forster, K., et al. 2017, AJ, 153, 2

Maraschi, L. and Fossati, G. and Tavecchio, F. et al. 1999, ApJL, 526, L81

Marscher, A. P. 2014, ApJ, 780, 87

Mastichiadis, A. and Petropoulou, M. and Dimitrakoudis, S. 2013, MNRAS, 434, 2684

Moraitis, K. \& Mastichiadis, A. 2011, A\&A, 525, A40

Paliya, V. S., Böttcher, M., Diltz, C., et al. 2015, ApJ, 811, 143 
Paliya, V. S., Marcotulli, L., Ajello, M., et al. 2017, ApJ, 851, 33

Paneque, D., D'Ammando, F., Orienti, M., \& Falcon, A. 2013, The Astronomer's Telegram, 4977

Petropoulou, M. 2014, A\&A, 571, 83

Petropoulou, M., Giannios, D., \& Sironi, L. 2016, MNRAS, 462, 3325

Petropoulou, M., \& Sironi, L. 2018, MNRAS, 481, 4

Piner, B. G., \& Edwards, P. G. 2005, ApJ, 622, 168

Piner, B. G., Unwin, S. C., Wehrle, A. E., et al. 1999, ApJ, 525,176

Poutanen, J., Zdziarski, A. A., \& Ibragimov, A. 2008, MNRAS, 389, 1427

Press, W. H., Teukolsky, S. A., Vetterling, W. T., \& Flannery, B. P. 2002, Numerical Recipes in C++ : the Art of Scientific Computing

Punch, M., Akerlof, C. W., Cawley, M. F., et al. 1992, Nature, 358, 477

Romanova, M. M. and Lovelace, R. V. E., 1992, A\&A, 262, 26

Schwarz, G. J., Ness, J.-U., Osborne, J. P., et al. 2011, ApJS, 197, 31

Sironi, L., Giannios, D., \& Petropoulou, M. 2016, MNRAS, 462,48
Sironi, L., Petropoulou, M., \& Giannios, D., 2015, MNRAS, 450, 183

Sironi, L., Giannios, D., \& Petropoulou, M., 2016, MNRAS, 462, 1

Tavecchio, F., Maraschi, L., \& Ghisellini, G. 1998, ApJ, 509,608

Tavecchio, F., \& Ghisellini, G. 2008, American Institute of Physics Conference Series, 431

Tavecchio, F., Ghisellini, G., Ghirlanda, G., Foschini, L., Maraschi, L. 2010, MNRAS, 401, 3

Tavecchio, F., \& Ghisellini, G. 2016, MNRAS, 456, 3

Ulrich, M.-H., Kinman, T. D., Lynds, C. R., Rieke, G. H., \& Ekers, R. D. 1975, ApJ, 198, 261

Vaughan, S., Edelson, R., Warwick, R. S., \& Uttley, P. 2003, MNRAS, 345, 1271

Vogt, F. P. A., Dopita, M. A., Kewley, L. J., et al. 2014, ApJ, 793, 127

Villata, M., Raiteri, C. M., Larionov, V. M., et al. 2008, A\&A, 481, L79

Villata, M., Raiteri, C. M., Gurwell, M. A., et al. 2009, A\&A, 504, L9

Zhang, H., Li, X., Guo, F., \& Giannios, D. 2018, ApJL, 862, 2 